
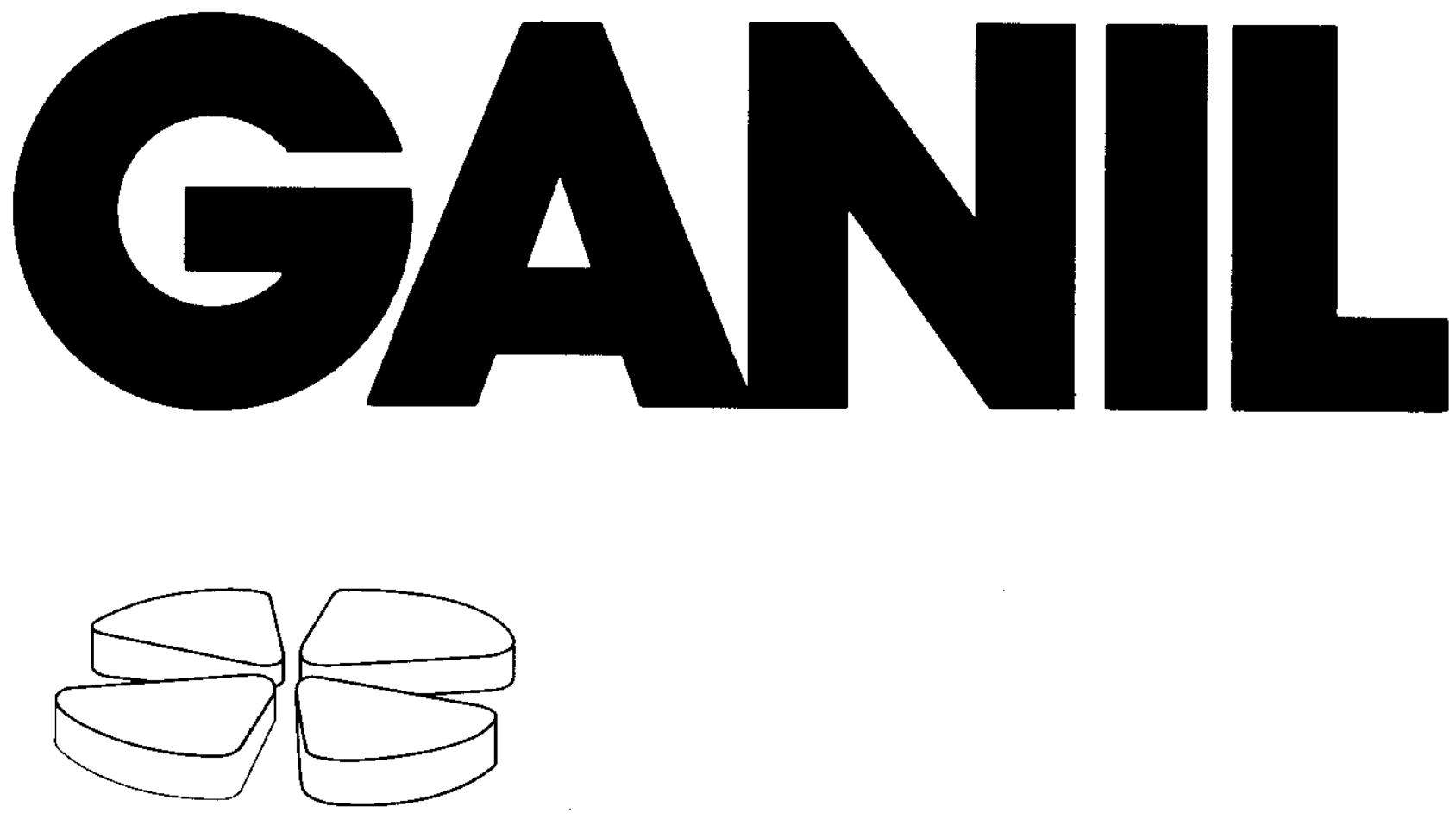

Phase Transitions in finite systems

Ph. Chomaz ${ }^{1}$ and F. Gulminelli ${ }^{2}$

1 GANIL (DSM-CEA/IN2P3-CNRS), B.P.5027, F-14021 Caen cedex, France

2 LPC Caen, (IN2P3-CNRS/ISMRA et Université), F-14050 Caen cedex, France

CERN LIBRARIES, GENEVA

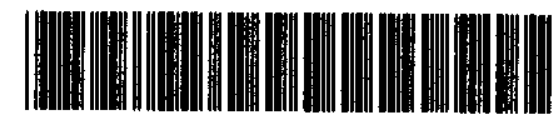

CM-P00040576 


\title{
Phase Transitions in finite systems
}

\author{
Ph. Chomaz ${ }^{1}$ and F. Gulminelli ${ }^{2}$ \\ ${ }^{1}$ GANIL (DSM-CEA/IN2P3-CNRS), B.P.5027, F-14021 Caen cedex, France \\ 2 LPC Caen, (IN2P3-CNRS/ISMRA et Université), F-14050 Caen cedex, France
}

\begin{abstract}
In this series of lectures we will first review the general theory of phase transition in the framework of information theory and briefly address some of the well known mean field solutions of three dimensional problems. The theory of phase transitions in finite systems will then be discussed, with a special emphasis to the conceptual problems linked to a thermodynamical description for small, short-lived, open systems as metal clusters and data samples coming from nuclear collisions. The concept of negative heat capacity developed in the early seventies in the context of self-gravitating systems will be reinterpreted in the general framework of convexity anomalies of thermostatistical potentials. The connection with the distribution of the order parameter will lead us to a definition of first order phase transitions in finite systems based on topology anomalies of the event distribution in the space of observations. Finally a careful study of the thermodynamical limit will provide a bridge with the standard theory of phase transitions and show that in a wide class of physical situations the different statistical ensembles are irreducibly inequivalent.
\end{abstract}

\section{Equilibrium and Information}

\subsection{States and observables [2,3]}

Modern physics associates to every physical system two different types of objects: observables that characterize the measurable physical quantities and states whose knowledge allows to predict the result of experiments. From the microscopic point of view, single realizations of systems with $N$ degrees of freedom are characterized by a pure state (or microstate), that is a wave function, $\left|\Psi_{N}\right\rangle$, in quantum mechanics or a point in the 2N-dimensional phase space, $\mathbf{s}=\left(q_{1}, q_{2}, \ldots, q_{N} ; p_{1}, p_{2}, \ldots, p_{N}\right)$, with $q_{i}$ and $p_{i}$ the position and momentum of each degree of freedom, in classical mechanics. If systems are sufficiently complex, the exact state is in general impossible to define and each actual realization corresponds to a microstate $(n)$ with the probability $p^{(n)}$. In such a realistic case, one rather speaks of mixed states (or macrostates) described the density

$$
\widehat{D}=\sum_{n}\left|\psi^{(n)}\right\rangle p^{(n)}\left\langle\psi^{(n)}\right| \text { or } \widehat{D}(\mathbf{s})=\sum_{n} \delta\left(\mathbf{s}-\mathbf{s}^{(n)}\right)
$$

Observables are operators defined on the Hilbert space or classically real functions of $2 \mathrm{~N}$ real variables. The information that can be associated to the system is the ensemble of expectation values of the observables $\widehat{A}_{l}$ on the 
state, i.e. the ensemble of observations $\left\langle\widehat{A}_{l}\right\rangle=\sum_{n} p^{(n)} A_{l}^{(n)}$ where $A_{l}^{(n)}$ is the actual result of a measurement on the realization $(n)$. In the quantum case $\left\langle\widehat{A}_{l}\right\rangle=\sum_{n} p^{(n)}\left\langle\psi^{(n)}\left|\widehat{A}_{l}\right| \psi^{(n)}\right\rangle=\operatorname{Tr}\left(\widehat{D} \hat{A}_{l}\right)$. Both for pure and mixed states, if the information on the system is complete at the initial time, this stays true at any time because the dynamical evolution of states is governed by the deterministic Liouville Von Neumann equation $\partial_{t} \widehat{D}=\{\widehat{H}, \widehat{D}\}$ where $\widehat{H}$ is the Hamiltonian of the system and where $\{.,$.$\} is the commutator divided by i \hbar$ in quantum mechanics which reduces to the usual Poisson bracket at the classical limit. However in the case of complex systems, the initial conditions are in general incompletely known and an exact solution of the Liouville Von Neumann equation is out of reach. In general only a small set of pertinent observables is known at any time which is sufficient to determine the state (i.e. the totality of the $p^{(n)}$ ) because of the complexity of the density operator.

\subsection{The Shannon entropy $[2,3]$}

The incompleteness of the available information can be measured through the lack of information or statistical entropy

$$
S=-\sum_{n} p^{(n)} \ln p^{(n)}=-\operatorname{Tr}(\widehat{D} \ln \widehat{D})
$$

Let us show within a simple example that the statistical entropy (or Shannon entropy) indeed measures the lack of information.

Let us consider a system constituted of $N$ identical boxes and an experiment consisting in putting randomly a ball in a box. The missing information $S$ to know where the ball is depends first on the occupation probability of each box $S=S\left(p^{(1)}, \ldots, p^{(N)}\right)$. Let us first consider equiprobable boxes $p^{(n)}=1 / N, \forall n$. In this case $S$ depends only on the total number of boxes, $S=S(N)$.

Let us enumerate some fundamental properties of $S$ :

- The lack of information must grow with the number of possible results $S\left(N_{1}\right)>S\left(N_{2}\right) \forall N_{1}>N_{2}$.

Let us divide the $N$ boxes into $N_{1}$ groups of $N_{2}$ boxes each, $N=N_{1} N_{2}$. The experiment now consists in two successive steps, first find out in which group out of the $N_{1}$ equiprobable ones the ball is (which is associated to a lack of information $S\left(N_{1}\right)$ ) and then determine which of the $N_{2}$ equiprobable boxes belonging to the group the ball is (associated to a lack of information $S\left(N_{2}\right)$ ). The missing information of the two steps experiment is then $S\left(N_{1}\right)+S\left(N_{2}\right)$.

- The information cannot depend on the number of steps through which it is collected $S(N)=S\left(N_{1} \cdot N_{2}\right)=S\left(N_{1}\right)+S\left(N_{2}\right)$. 
The ensemble of these properties is fulfilled by a logarithmic function $S(N)=$ $k \ln N$ where $k$ is a constant.

We have just shown that the Shannon entropy coincides for equiprobable states with the Boltzmann entropy (or microcanonical entropy). Let us now turn to the more general case in which boxes are not equiprobable. To derive the associated information let us consider a big number $W$ (eventually going to infinity) of experiments identical to the one described above. Among these $W$ experiences, a number $N_{i} \neq W / N$ will lead to the observation of the ball in the $i$-th box. This experimental result defines a posteriori a probability $p_{i}=N_{i} / W$ for the $i$-th box.

Within this result $\left\{N_{1}, \ldots, N_{k}, \ldots, N_{N}\right\}$, the number of possible configurations for the box is given by the combinatorial

$$
\Omega=\frac{W !}{N_{1} !\left(W-N_{1}\right) !} \cdot \frac{\left(W-N_{1}\right) !}{N_{2} !\left(W-N_{1}-N_{2}\right) !} \cdots=\frac{W !}{\prod_{i} N_{i} !}
$$

where the first term represents the number of ways of choosing $N_{1}$ indistinguishable objects out of $W$, and so on. All the events $\Omega$ are equiprobable. The entropy is then

$$
\begin{aligned}
S(\Omega) & =k \ln \Omega=k\left(\ln W !-\sum_{i} \ln N_{i} !\right) \\
& =k\left(W \ln W-W-\sum_{i}\left(N_{i} \ln N_{i}-N_{i}\right)\right) \\
& =k\left(W \ln W-\sum_{i}\left(W p_{i} \ln W+W p_{i} \ln p_{i}\right)\right) \\
& =-k W \sum_{i} p_{i} \ln p_{i}
\end{aligned}
$$

where we have used the Stirling formula $\ln N ! \approx N \ln N-N$. The additivity property introduced above allows to conclude that for a single experiment the missing information is given by the Shannon entropy $S\left(p^{(1)}, \ldots, p^{(N)}\right)=$ $-k \sum_{n} p^{(n)} \ln p^{(n)}$.

It may be interesting to know that if the additivity property of the information is relaxed, it is possible to construct a non-extensive extension of the Shannon theory based on the so called q-statistics which has interesting applications in out of equilibrium situations as in the case of turbulent flows [4]. In the following of these lectures we will limit ourselves to the standard information kernel introduced above.

\subsection{The fundamental postulate of statistical mechanics}

The fundamental postulate of statistical mechanics can be expressed as follows

"The statistical distribution of microstates usually called the equilibrium is the one which maximizes the statistical entropy within the external constraints (i.e. the pertinent information) imposed to the system". 
Indeed any other distribution would introduce an extra piece of information, in contrast with the statement that all the available information is given by the constraint.

It is important to remark that this postulate, though certainly intuitive and elegant, does not necessarily imply that the theory has any predictive power: the fact that we have only a limited amount of information on a system does not necessarily mean that the information contained in the system is objectively limited. In this series of lectures we shall anyway keep the fundamental postulate as the only reasonable working hypothesis in a complex system.

The fundamental postulate of statistical mechanics allows to determine the equilibrium values of the state probabilities $p^{(n)}$. This task is easily accomplished with the help of the method of Lagrange multipliers.

\subsection{The method of Lagrange multipliers[5]}

Let us consider the problem of finding an extremum of a two variables real function $f(x, y)$ along a curve defined by the relation $\omega(x, y)=\omega_{0}$. To this aim the standard way is to calculate the total differential

$$
d f=\frac{\partial f}{\partial x} d x+\frac{\partial f}{\partial y} d y
$$

where $d x$ and $d y$ are linked by the relation

$$
d \omega=\frac{\partial \omega}{\partial x} d x+\frac{\partial \omega}{\partial y} d y=0
$$

Expressing $d y$ as a function of $d x$ the differential reads

$$
d f=\left(\frac{\partial f}{\partial x}-\frac{\partial \omega / \partial x}{\partial \omega / \partial y} \frac{\partial f}{\partial y}\right) d x
$$

Putting $d f$ to zero at the point $\left(x_{0}, y_{0}\right)$ which fulfills the constraint $\omega\left(x_{0}, y_{0}\right)=$ $\omega_{0}$, leads to

$$
\left.\left.\frac{\partial \omega}{\partial y} \frac{\partial f}{\partial x}\right\rfloor_{x_{0}, y_{0}}=\frac{\partial \omega}{\partial x} \frac{\partial f}{\partial y}\right\rfloor_{x_{0}, y_{0}}
$$

which defines the coordinates $\left(x_{0}, y_{0}\right)$ of the extremum.

This same result can be obtained in a simpler way if we introduce a Lagrange multiplier $\lambda$ and we define the auxiliary function $F=f-\lambda\left(\omega-\omega_{0}\right)$ that coincides with the function $f$ on the curve we are interested in. Differentiating $F$ respect to its two independent variables $x$ and $y$

$$
d F=\left(\frac{\partial f}{\partial x}-\lambda \frac{\partial \omega}{\partial x}\right) d x+\left(\frac{\partial f}{\partial y}-\lambda \frac{\partial \omega}{\partial y}\right) d y
$$

the two partial derivatives have to go to zero separately at the extremum leading to a solution $\left(x_{0}(\lambda), y_{0}(\lambda)\right)$. This extremum fulfills the condition 


$$
\left.\left.\frac{\partial \omega}{\partial y} \frac{\partial f}{\partial x}\right\rfloor_{x_{0}, y_{0}}=\frac{\partial \omega}{\partial x} \frac{\partial f}{\partial y}\right\rfloor_{x_{0}, y_{0}}
$$

which exactly corresponds to the condition above if $\lambda$ is such that $\omega\left(x_{0}(\lambda)\right.$, $\left.y_{0}(\lambda)\right)=\omega_{0}$. The extension to a bigger number of variables and constraints is straightforward.

To summarize, this method allows to replace the study of a function of non independent variables to the study of an auxiliary function for which all variables are independent and the constraints are absorbed by real numbers (Lagrange multipliers).

\subsection{The equilibrium [5]}

Let us use this method to maximize the statistical entropy $S=-\operatorname{Tr} \hat{D} \ln \hat{D}$ under the constraint of a given set of $L$ observations $\left\langle\hat{A}_{l}\right\rangle$.

This situation corresponds to the $L$ constraints $\operatorname{Tr} \hat{D} \hat{A}_{l}=\left\langle\hat{A}_{l}\right\rangle$ that has to be augmented with the extra constraint of the normalization of probability $\operatorname{Tr} \hat{D}=1$ which can be incorporated as an additional observable $\hat{A}_{0}=1$. The auxiliary function is defined as

$$
Y=-\operatorname{Tr} \hat{D} \ln \hat{D}-\sum_{l=0}^{L} \lambda_{l} \operatorname{Tr} \hat{D} \hat{A}_{l}
$$

The variation of $Y$ induced by a variation $\delta \hat{D}$ of the density matrix $\hat{D}$ reads

$$
\delta Y=-\operatorname{Tr} \delta \hat{D}\left(\ln \hat{D}+1+\sum_{l=0}^{L} \lambda_{l} \hat{A}_{l}\right)
$$

The extremum correspond to $\delta Y=0$, with no restrictions on $\delta \hat{D}$ leading to the condition $\ln \hat{D}+1+\sum_{l=0}^{L} \lambda_{l} \hat{A}_{l}=0$. The solution is the density matrix at equilibrium which is a function of the Lagrange multipliers $\lambda_{l}$

$$
\hat{D}^{0}=\frac{1}{Z} \exp -\sum_{l=1}^{L} \lambda_{l} \hat{A}_{l}
$$

where we have already taken care of the normalization constraint by introducing the partition sum

$$
Z=\operatorname{Tr} \exp -\sum_{l=1}^{L} \lambda_{l} \hat{A}_{l}
$$


The link between a constraint $\left\langle\hat{A}_{i}\right\rangle$ (or observation, or extensive variable) and the associated Lagrange multiplier $\lambda_{l}$ (or thermodynamically conjugated intensive variable) is given by an equation of state

$$
\left\langle\hat{A}_{l}\right\rangle=-\frac{\partial \ln Z}{\partial \lambda_{l}}
$$

It is also possible to express $\lambda_{l}$ as a function of $\langle A\rangle$ by inverting the equation of state. Indeed the equilibrium corresponding to the considered constraints is associated to a value for the statistical entropy

$$
S=\operatorname{Tr} \hat{D}^{0} \ln \hat{D}^{0}=\sum_{l} \lambda_{l}\left\langle\hat{A}_{l}\right\rangle+\ln Z
$$

This last equation known as a Legendre transform gives the relation between the entropy and the partition sum and implies for the Lagrange multipliers

$$
\lambda_{l}=\frac{\partial S}{\partial\left\langle\hat{A}_{l}\right\rangle}
$$

It should be noticed that while $D^{0}$ and $Z$ are functions of the intensive variables $\left(\lambda_{l}\right)$, the Legendre transform $S$ is a function of the associated extensive variables $\left\langle\hat{A}_{l}\right\rangle$.

Using eqs. $(2,3,4)$ the whole thermodynamics of the system can be calculated if the constraints $\left\langle\hat{A}_{l}\right\rangle$ are known. It is important to remark that this formalism is completely general in the sense that it can be applied for an arbitrary number of bodies with no need of a thermodynamical limit (infinite systems), and that all observables (and not only variables conserved by the dynamics) can play the role of constraints. Moreover the maximization of entropy as a tool to deal with the general problem of missing information can be extended in dynamical situations and has shown to be a fruitful approach in the field of stochastic quantum transport [6].

\subsection{The usual thermodynamics [5]}

The usual ensembles of standard thermodynamics can also be obtained as applications of this general theory. Let us consider for example the case where the only constraint is the energy $\langle E\rangle=\operatorname{Tr}\left(\hat{D}^{0} \hat{H}\right)=\sum_{n} p_{n} E^{(n)}$ associated with the Lagrange multiplier $\beta$. The probability of the $\mathrm{n}$-th energy eigenstate is then $p_{n}^{0}=\frac{1}{Z_{\beta}} \exp \left(-\beta E^{(n)}\right)$ while the energy probability distribution reads $p_{\beta}(E)=\frac{W(E)}{Z_{\beta}} \exp (-\beta E)$ where $W(E)$ is the number of states corresponding to an energy $E$. The Lagrange multiplier $\beta$ has the physical meaning of the inverse of the temperature $T=\beta^{-1}$. The relation between the average energy and the 
temperature is given by the equation of state $\langle E\rangle=-\partial_{\beta} \ln Z_{\beta}$ and the Legendre transform $S(\langle E\rangle)=\ln Z_{\beta}+\beta\langle E\rangle$ represents the well known relation between the canonical entropy and the free energy $F_{T}=-\beta^{-1} \ln Z_{\beta}$.

The microcanonical ensemble can also be obtained from this general theory considering that in the absence of any constraint (except the normalization of probabilities) all states must be equiprobable. The microcanonical entropy is then obtained as the expression of the Shannon entropy within the equilibrium distribution $p_{i}^{0}=1 / W(E), S(E)=-\sum_{i=1}^{W} W^{-1} \ln W^{-1}=\ln W$.

\section{Generalities about phase transitions}

Generally speaking, for a given value of the control parameters (or intensive variables) $\lambda_{l}$, the properties of a substance are univocally defined, i.e. the conjugated extensive variables $\left\langle\hat{A}_{l}\right\rangle$ have a unique value unambiguously defined by the corresponding equation of state. For instance the volume occupied by $n$ moles of an ideal gas at a given pressure $P$ and temperature $T$ is given by $V=n R T / P$. In reality we have seen in the previous chapter that extensive variables, being by definition expectation values of operators, are associated with a probability distribution unless the system is described by a pure state. The intuitive expectation that extensive variables at equilibrium have a unique value therefore means that the probability distribution is narrow and normal, such that a good approximation can be obtained by replacing the distribution with its most probable value.

In this case, as we will see in section 2.1, the Legendre transform gives an exact mapping between the standard intensive ensembles in which the control parameter is intensive or equivalently only the average of the extensive variable is known and the more exotic extensive ensembles where an extensive variable is controlled event by event, demonstrating the equivalence between the different statistical ensembles. In the following we will often take as an paradigm of intensive ensembles the canonical ensemble for which the inverse of the temperature $\beta^{-1}$ (or equivalently the average energy $\langle E\rangle$ ) is controlled while the archetype of the extensive ensemble will be the microcanonical one for which the energy is strictly controlled.

The normality of probability distributions is usually assumed on the basis of the central limit theorem that we will briefly review in section 2.2 . However some situations exist in which the probability distributions of extensive variables are abnormal and for example bimodal: in this case two different properties (phases) coexist for the same value of the intensive control variable. A first elementary description of phase coexistence using this intuitive bimodality argument will be given at the end of section 2.2 .

The topological anomalies of probability distributions and the failure of the central limit theorem in phase coexistence imply that in a first order phase transition the different statistical ensembles are in general not equivalent and different phenomena can be observed depending on the fact that the controlled variable is extensive or intensive. This general statement will be developed in 
great detail in chapter 4 and its far reaching consequences will be analyzed in chapter 6 .

\subsection{The difference between Laplace and Legendre}

We have seen in the last chapter that the relation between the different thermostatistical potentials is given by the Legendre transform. It is important to distinguish between transformations within the same statistical ensemble as the Legendre transform (which gives for instance the link between the canonical partition sum and the canonical entropy) and transformations between different ensembles which are instead given by non linear integral transforms. Let us consider energy as the extensive observable and temperature as the conjugated intensive one. The definition of the canonical partition sum is

$$
Z_{\beta}=\sum_{n} \exp \left(-\beta E^{(n)}\right)
$$

where the sum runs over the available eigenstates $n$ of the Hamiltonian. If energy can be treated as a continuum variable this equation can be written as

$$
Z_{\beta}=\int_{0}^{\infty} d E W(E) \exp (-\beta E)
$$

which is nothing but a Laplace transform between the canonical partition sum and the microcanonical entropy $S_{E}=\ln W(E)$. If the integrand $f(E)=W(E) \exp (-\beta E)$ is a strongly peaked function the integral can be replaced by the maximum $f(\bar{E})$

$$
Z_{\beta} \approx W(\bar{E}) \exp (-\beta \bar{E})
$$

which can be rewritten as

$$
\ln Z_{\beta} \approx S_{\bar{E}}-\beta \bar{E}
$$

or introducing the free energy $F_{T}=-\beta^{-1} \ln Z_{\beta}$

$$
F_{T} \approx \bar{E}-T S_{\bar{E}}
$$

Eq.(9) has the structure of an approximate Legendre transform and shows that in the saddle point approximation eq.(8) the ensembles differing at the level of constraints acting on a specific observable (here energy) differ only by a simple linear transformation. We will see in the next section and in more details in chapter 6 that however the saddle point approximation eq. (8) can be highly incorrect close to a phase transition. In particular, when the canonical distribution of energy is bimodal a unique saddle point approximation becomes inadequate. In this case eq.(9) cannot be applied and eq.(7) is the only possible transformation between the different ensembles.

To summarize one should not confuse 
- the link between the thermodynamical potential of the intensive (e.g. log of canonical partition sum) and of the extensive ensemble (e.g. the microcanonical entropy) which are always related with a Laplace transform. This Laplace transform may lead to an approximate Legendre transformation for normal distributions but we know that this Legendre transformation is wrong if the distribution is abnormal.

- with the exact Legendre transform between the entropy of the intensive ensemble and the corresponding thermodynamical potential.

This simply corresponds to the fact that the microcanonical and canonical entropies can be very different.

\subsection{The central limit theorem and phase coexistence}

The typical representation of the probability distribution of any generic random variable depending on a not too small number of degrees of freedom is a Gaussian distribution. The very general validity of the Gaussian is due to one of the most important theorems of statistics, the Laplace central limit theorem. Let us consider an extensive observable $E$ (i.e., energy) that can be written as the sum of $I$ independent contributions (i.e. the energy of the different particles constituting the system) $E=\sum_{i}^{I} e_{i}$, where the $e_{i}$ follow an arbitrary probability distribution with the unique requirement that the global variance $\sigma_{E}^{2}=\sum_{i}^{I}\left(\left\langle e_{i}^{2}\right\rangle-\left\langle e_{i}\right\rangle^{2}\right) / I$ is finite. Then the central limit theorem states that the distribution of $E$ tends to a Gaussian distribution with a width decreasing with the number of degrees of freedom

$$
\lim _{I \rightarrow \infty} p(E)=\frac{1}{\sqrt{ } 2 \pi \sigma^{2} / l} \exp \left(-\frac{(E-\langle E\rangle)^{2}}{2 \sigma^{2} / I}\right)
$$

According to the central limit theorem at the thermodynamical limit the distribution of an extensive variable $p(E)$ tends to a $\delta$-function, implying as we have mentioned at the beginning of the chapter that the properties of the system are univocally defined by the value of the intensive parameter that controls the asymptotic value of $\langle E\rangle$ through the appropriate equation of state. Moreover in most cases a few tens of particles are enough for the Gaussian approximation to be correct, meaning that the limit appearing in eq.(10) can be neglected in practical applications. Another consequence of the central limit theorem is that the Laplace transform becomes equivalent to a Legendre transform as we have discussed in the preceding section, leading to the equivalence of statistical ensembles.

However a situation can occur in which the probability distribution is bimodal and never tends to a Gaussian. Such a situation is called a first order phase transition. This patent violation of the central limit theorem is due to the fact that phase transitions are associated to long range correlations and the independence hypothesis between the different degrees of freedom breaks down.

Let us illustrate the standard picture of phase coexistence within a simple example. Consider a molecular system in the canonical ensemble characterized by 
the free energy $F=-T \ln Z=\langle E\rangle-T S$. As we have demonstrated in section 1.3 the maximization of the statistical entropy with the energy constraint is equivalent to the minimization of the free energy. At low temperature a minimization of $F$ is approximately equivalent to a minimization of $\langle E\rangle$ : the equilibrium state of the system will be given by a compact configuration (a crystal or a liquid) with free energy $F_{L}(A, V)$. On the other side at high temperature the minimization of $F$ corresponds to a maximization of the canonical entropy, which will be achieved by a disordered rarefied state (a gas phase) with free energy $F_{G}(A, V)$. Phase coexistence means that at an intermediate temperature the two free energy solutions are allowed giving for the total free energy

$$
F(A, V)=F_{L}\left(A_{L}, V_{L}\right)+F_{G}\left(A_{G}, V_{G}\right)
$$

where $A_{L}, V_{L}\left(A_{G}, V_{G}\right)$ are the fractions of total number of molecules $A$ and volume $V$ belonging to the ordered (disordered) phase

$$
A=A_{L}+A_{G} \quad ; \quad V=V_{L}+V_{G}
$$

The equilibrium sharing of $A$ and $V$ is given by the minimization of the free energy

$$
\frac{\partial F}{\partial A_{L}}=\frac{\partial F_{L}}{\partial A_{L}}-\frac{\partial F_{G}}{\partial A_{G}}=0 \quad ; \quad \frac{\partial F}{\partial V_{L}}=\frac{\partial F_{L}}{\partial V_{L}}-\frac{\partial F_{G}}{\partial V_{G}}=0
$$

implying the equality for the intensive variables conjugated to the mass number and the volume, namely the chemical potential and the pressure

$$
p_{L}=p_{G} \quad ; \quad \mu_{L}=\mu_{G}
$$

This procedure can be generalized to any statistical ensemble. If we consider for example the microcanonical ensemble, the absence of constraints means that the thermostatistical potential is directly the microcanonical entropy

$$
S(A, E, V)=\ln W(A, E, V)=S_{L}\left(A_{L}, E_{L}, V_{L}\right)+S_{G}\left(A_{G}, E_{G}, V_{G}\right)
$$

with the extra conservation law $E=E_{L}+E_{G}$. The extremization of $S$ respect to $V$ and $A$ gives again the equality of the chemical potential and pressure for the two coexisting phases, while the derivative respect to the energy variable gives

$$
T_{L}=T_{G}
$$

where we have defined the microcanonical temperature as $T^{-1}=\partial_{E} S$ in analogy with the canonical Legendre transform $\beta=\partial_{\langle E\rangle} S_{\beta}$ (the justification of the physical meaning of $\partial_{E} S$ as an inverse temperature is postponed to chapter 4).

Equilibrium between the two phases is characterized by the equality of the temperatures. On the other hand, the conjugated extensive variables are different in the two phases $E_{L}<E_{G}$. This means that at the transition temperature $T_{t r}=T_{L}=T_{G}$ the energy is discontinuous at the phase transition (latent heat).

To summarize, in this standard view first order phase transitions are characterized by 
- the presence of two phases in contact

- a discontinuity in (one ore more) first order derivatives of the thermostatistical potential (energy, volume, mass number...).

To obtain this result we have written the thermostatistical potential as a simple sum of the contributions of the two phases (eqs.(11,12)). This is true only if the free energy (or entropy) of the interface between the two phases is negligible, i.e. for large systems interacting through short range forces.

In the next sections we will illustrate this standard view of first order phase transitions within an exactly solvable model in one and two dimensions (section $2.3,2.4$ ) and in three dimensions with the help of the mean field approximation (chapter 3).

The additivity hypothesis of the thermostatistical potential breaks down for finite systems and even in the thermodynamical limit if the forces are long ranged. The far reaching consequences of dropping this approximation will be developed in chapter 4 .

\subsection{Isomorphism between Ising and Lattice Gas}

Let us consider an ensemble of $N$ classical spins which can take one of the two values $s_{k}= \pm 1$ on a lattice under the influence of an external magnetic field $h$ and a constant coupling $J$ between neighboring sites according to the Hamiltonian

$$
H_{I S}=-h \sum_{k=1}^{N} s_{k}-\frac{J}{2} \sum_{k \neq j}^{N} s_{k} s_{j}
$$

where the second sum extends over closest neighbors.

The Ising model eq.(13) has been originally introduced to give a simple description of ferromagnetism (i.e. a spontaneous magnetization that some substances present in the absence of a field at low temperature). In reality the phenomenon of ferromagnetism is far too complicated to be treated in a satisfactory way by this oversimplified Hamiltonian; however the fact that the Ising model is exactly solvable in $1 \mathrm{D}$ and $2 \mathrm{D}$ and that very accurate numerical solutions exist for the three dimensional case makes this model a paradigm of first and second order phase transitions. The other appealing side of the Ising model is its versatility: introduced to explain magnetic phase transitions, it is also well adapted to describe fluid phase transitions. Indeed we can show that a close link exists between the Ising Hamiltonian eq.(13) and the Lattice Gas Hamiltonian which is the simplest modelization of the liquid-gas phase transition

$$
H_{L G}=\frac{1}{2 m} \sum_{k=1}^{N} \boldsymbol{p}_{k}^{2} n_{k}-\frac{\varepsilon}{2} \sum_{k \neq j}^{N} n_{k} n_{j}
$$

In the Lattice Gas model, the same $N$ lattice sites in $D$ dimensions are characterized by an occupation $n_{k}=0,1$ and by a $D$ components momentum 
vector $\boldsymbol{p}_{k}$. Occupied sites (particles) interact with a constant closest neighbor coupling $\varepsilon$.

Because of the transformation $n_{k}=\left(s_{k}+1\right) / 2$ the Ising Hamiltonian $H_{I S}$ can be mapped into the interaction part $H_{L G}^{\text {int }}$ of the Lattice Gas Hamiltonian $H_{L G}$. Indeed let us consider the interaction part of the Lattice Gas partition sum in the grancanonical ensemble

$$
Z_{L G}^{i n t}=\sum_{n_{1}=0,1} \ldots . . \sum_{n_{N}=0,1} \exp \left(-\beta\left(H_{L G}^{i n t}-\mu A\right)\right)
$$

where $A=\sum_{k}^{N} n_{k}$ is the total number of particles and $\beta, \mu$ are Lagrange multipliers. The factor multiplied by $-\beta$ in the exponential can be written as

$$
H_{L G}^{i n t}-\mu A=-\frac{\varepsilon}{8} \sum_{k \neq j}^{N} s_{k} s_{j}-\left(\frac{\varepsilon z+2 \mu}{4}\right) \sum_{i=1}^{N} s_{i}-N\left(\frac{\varepsilon z+4 \mu}{8}\right)
$$

where $z=2 D$ is the number of closest neighbors. With the identification $J=$ $\varepsilon / 4$ and $h=(z \varepsilon+2 \mu) / 4$, this equation shows that the grancanonical partition sum of the Lattice Gas interaction hamiltonian is isomorphous to the canonical partition sum of the Ising model in an external field

$$
\beta\left(H_{L G}^{i n t}-\mu A\right)=\beta H_{I S}+K
$$

where $K$ is a constant. This result implies that all results obtained within the Ising model concerning magnetic transitions can be translated in terms of fluid transitions and vice-versa. In particular the magnetization $m=\left\langle\sum_{k} s_{k}\right\rangle / N$ is related to the matter density $\rho=\left\langle\sum_{k} n_{k}\right\rangle / N$ by $m=2 \rho-1$.

\subsection{Exact solution of the Ising model in 1D and 2D}

The Ising model was proposed by Lenz to his student Ising in 1925. The exact solution of the model in one dimension is given in Ising's thesis.

Let us consider a one dimensional spin chain with periodic boundary conditions (spin ring). The Ising hamiltonian can be written as

$$
H_{I S}=-\sum_{k=1}^{N}\left(h s_{k}+J s_{k} s_{k+1}\right)
$$

and the partition sum results

$$
Z_{I S}=\sum_{s_{1}=-1}^{1} \ldots . . \sum_{s_{N}=-1}^{1} \exp \left(\beta \sum_{k=1}^{N}\left(h s_{k}+J s_{k} s_{k+1}\right)\right)=\sum_{s_{1}=-1}^{1} \ldots . . \sum_{s_{N}=-1}^{1} \tau_{12} \tau_{23} \ldots \tau_{N 1}
$$

where we have introduced 


$$
\tau_{i j}=\exp \left(\frac{h}{2}\left(s_{i}+s_{j}\right)+J s_{i} s_{j}\right)
$$

If we consider the $\tau_{i j}$ as the elements of a $2 \times 2$ matrix depending upon the two spins $s_{i}= \pm$ and $s_{j}= \pm$

$$
T=\left(\begin{array}{l}
\tau_{+}+\tau_{+} \\
\tau_{-}+\tau_{--}
\end{array}\right)
$$

where the definition of the $\tau_{i j}$ implies

$$
\begin{aligned}
& \tau_{++}=\exp \beta(J+h) \\
& \tau_{--}=\exp \beta(J-h) \\
& \tau_{+-}=\tau_{-+}=\exp (-\beta J),
\end{aligned}
$$

then we can write $\sum_{s_{j}=-1}^{1} \tau_{i j} \tau_{j k}=T_{i k}^{2}$ and the partition sum becomes

$$
Z_{I S}=\sum_{s_{1}=-1}^{1} T_{11}^{N}=\operatorname{Tr}\left(T^{N}\right)=\lambda_{1}^{N}+\lambda_{2}^{N}=\lambda_{1}^{N}\left(1+\left(\frac{\lambda_{2}}{\lambda_{1}}\right)^{N}\right) \underset{N \rightarrow \infty}{\longrightarrow} \lambda_{1}^{N}
$$

where $\lambda_{1}, \lambda_{1}\left(\lambda_{1}>\lambda_{2}\right)$ are the eigenvalues of the $T$ matrix. The problem is then reduced to an eigenvalue problem

$$
\operatorname{det}(T-\lambda I)=0 \quad ; \quad \lambda^{2}-\left(\tau_{+}++\tau_{--}\right) \lambda+\left(\tau_{+}+\tau_{-}-\tau_{+}-\tau_{-}\right)=0
$$

After a little algebra we obtain the eigenvalues

$$
\lambda=\exp (\beta J)\left(\operatorname{ch}(\beta h) \pm\left(\exp (-4 \beta J)+\operatorname{sh}^{2}(\beta h)\right)^{\frac{1}{2}}\right)
$$

and the partition sum

$$
\ln Z_{I S}=N\left(\beta J+\ln \left(\operatorname{ch}(\beta h)+\left(\exp (-4 \beta J)+\operatorname{sh}^{2}(\beta h)\right)^{\frac{1}{2}}\right)\right)
$$

It is easy to verify that $\ln Z_{I S}$ is a continuous function with continuous derivatives for all orders: the Ising model in one dimension does not present a phase transition. In particular the magnetization

$$
m=\frac{1}{N \beta} \frac{\partial \ln Z_{I S}}{\partial h}=\frac{1}{2} \operatorname{sh}(\beta h)\left(\exp (-4 \beta J)+\operatorname{sh}^{2}(\beta h)\right)^{\frac{1}{2}}
$$

is a continuous monotonic function which is zero at zero field: no spontaneous magnetization is observed.

The solution of the Ising model in two dimensions[7] is far too complicated to be developed here. Let us simply give the asymptotic result $N \rightarrow \infty$ in the zero field case 

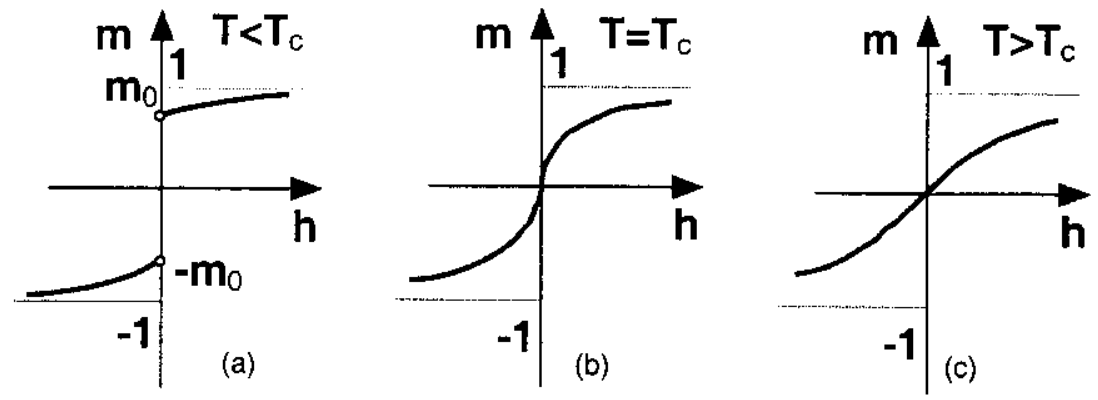

Fig. 1. Schematic representation of the average magnetization as a function of the applied external field for the Ising model in more than one dimension at subcritical (left), critical (center) and supercritical (right) temperature.

$$
\begin{aligned}
& Z_{I S}=(2 \operatorname{ch}(2 \beta J) \exp I)^{N} \\
& I=\frac{1}{2 \pi} \int_{0}^{\pi} d \phi \ln \frac{1+\left(1-x^{2} \sin ^{2} \phi\right)^{\frac{1}{2}}}{2} \\
& x=2 \frac{\operatorname{sh}(2 \beta J)}{\operatorname{ch}^{2}(2 \beta J)}
\end{aligned}
$$

With the partition sum of eq.(15) the magnetization equation of state can be computed. It is easy to verify that for temperatures lower than the critical temperature $T_{c}=\beta_{c}^{-1}$ given by $s h\left(2 J \beta_{c}\right)=1$ the system presents a spontaneous magnetization at zero field $[8]$

$$
m_{0}=m\left(h=0, \beta>\beta_{c}\right)=\frac{c^{2}(2 \beta J)}{s h^{4}(2 \beta J)}\left(s h^{2}(2 \beta J)-1\right)^{1 / 8} \underset{\beta \rightarrow \beta_{c}}{\longrightarrow}\left(T_{c}-T\right)^{1 / 8}
$$

The equation of state of the Ising model in more than one dimension is schematically shown in figure 1 .

At subcritical temperatures a discontinuity in magnetization is seen at zero field, showing that a first order phase transition is taking place in agreement with the intuitive arguments of the preceding section. For $T=T_{c}$ the magnetization goes to zero as a power law (second order phase transition) while the equation of state is monotonous in the supercritical regime.

\section{The mean field approximation}

Even for simplified models such as Ising no analytical solution exists for a number of dimension $D>2$. This is the reason why mean field solutions have been developed. The idea of the mean field approximation is to replace the intractable 
$\mathrm{N}$-body problem with an approximately equivalent analytical one body problem. Let us illustrate this method on the Ising case. If the Hamiltonian is composed of one body terms solely

$$
H_{1 b}=-\sum_{k=1}^{N} h_{k} s_{k}
$$

with $h_{k}$ a generic one body operator, the thermodynamics of the system is solved in one line. Indeed the partition sum in the canonical ensemble reads

$$
Z_{1 b}=\sum_{s_{1}=-1}^{+1} \ldots \sum_{s_{N}=-1}^{+1} \exp \left(-\beta H_{1 b}\right)=\prod_{k=1}^{N} z_{k}=(\exp (-\beta h)+\exp (\beta h))^{N}
$$

where the last equality holds if $h_{k}=h \forall k$, and is promptly generalized to the non-local case.

To reduce the Hamiltonian to a one body interaction the correlations among the different sites have to be neglected such that the interaction on a given site depends only of the coordinates of the site. This chapter is devoted to the applications of this approximation to the Ising model (section 3.1) and its general consequences for the problem of first order phase transitions (sections 3.2-3.3). We will see that an equivalent one body problem can be formulated and the two body character of the force results in a self-consistency problem for the equations of state which have to be solved iteratively.

It is important to stress that all mean field approaches are approximations which, because of the intrinsic lack of correlations, are especially bad in phase coexistence. In the recent years the enormous progress of computing machines has allowed the numerical solution of three dimensional models without any approximation with Monte-Carlo based methods. These exact solutions clearly show the inherent limitations of mean field approaches and will be discussed in chapter 4.

\subsection{Mean field approximation for the Ising model}

The interaction acting on the $k$-th site in the Ising model eq.(13) is $h_{k}=h+$ $J \sum_{j} s_{j}$, where the sum extends over the first neighbors of site $k$. A one body term is obtained if the spin of the neighboring sites $s_{j}$ is assumed constant all over the lattice and equal to the average magnetization $s_{j} \approx\langle s\rangle=m$. In other words the exact interaction is approximated by the interaction the site would experience if the spin distribution was uniform. The Ising Hamiltonian can then be written as a one body Hamiltonian

$$
H_{M F}=-\sum_{k=1}^{N} h_{k} s_{k}+K=-\sum_{k=1}^{N}(h+J z m) s_{k}+K
$$


within a constant $K$ which has to be determined by imposing that the expectation value of $H_{M F}$ is equal to the mean field energy

$$
E_{M F}=-h N m+E_{M F}^{i n t}=-N\left(h m+\frac{J z}{2} m^{2}\right)
$$

where the last equality is obtained by writing the interaction energy as

$$
E^{i n t}=-\frac{J}{2} \sum_{k=1}^{N} \sum_{j \neq k}\left\langle s_{k} s_{j}\right\rangle \approx-\frac{J}{2} \sum_{k=1}^{N} \sum_{j \neq k}\left\langle s_{k}\right\rangle\left\langle s_{j}\right\rangle=-\frac{J}{2} N z m^{2}
$$

which shows once again that the effect of the mean field approximation is the neglect of two body correlations. The comparison of eq.(19) with the expectation value of eq.(18) leads to the definition of the constant $K$ as $K=J N z m^{2} / 2$. In fact this energy correction exactly compensates the double counting of the twobody interaction due to the introduction of the average interaction of each spin with all its neighbors. The mean field partition sum as for eq.(17) is factorized in the product of the individual partition sums of the different sites

$$
Z_{M F}=\sum_{s_{1}= \pm 1} \ldots \sum_{s_{N}= \pm 1} \exp \left(-\beta\left(H_{M F}\right)\right)=z^{N}
$$

where

$z=\sum_{s= \pm 1} \exp \left(-\beta\left(-(h+J z m) s+\frac{J z}{2} m^{2}\right)\right)=2 \exp \left(-\beta \frac{J z}{2} m^{2}\right) \operatorname{ch}(\beta(h+J z m))$

which leads to a self-consistent equation for the magnetization

$$
m=\tanh (\beta(h+J z m))
$$

Equation (21) is represented in figure 2 in the subcritical, critical and supercritical regime. If the behavior of the equation of state for $T \geq T_{c}=J z$ is qualitatively similar to the exact Onsager solution of section 2.4, in the first order phase transition regime the mean field solution shows a backbending behavior with a negative susceptibility $\chi^{-1}=\partial_{h} m$ region. To understand the physical meaning of the backbending, the free energy $F=-\beta^{-1} \ln Z_{M F}$ is shown as a function of magnetization in figure 3 in the $h=0$ and $h>0$ case. From this figure one can see that the backbending corresponds to a maximum of the free energy, i.e. an instability. Indeed the coexistence between the two phases at different magnetization cannot be obtained in a mean field calculation because of the intrinsic homogeneity hypothesis $m=\langle s\rangle=$ const. The backbending therefore reflects the instability of the homogeneous mean field solution with zero magnetization respect to the separation into two distinct phases at $m= \pm m_{0}$. At non zero field the magnetization oriented in the direction of the field has the minimum free energy, therefore will correspond to the unique equilibrium solution. In the zero field case the two solutions have the same energy. This implies that every linear combination of these solutions

$$
m\left(h=0, T<T_{c}\right)=\alpha m_{0}+(1-\alpha)\left(-m_{0}\right) \quad ; \quad 0<\alpha<1
$$



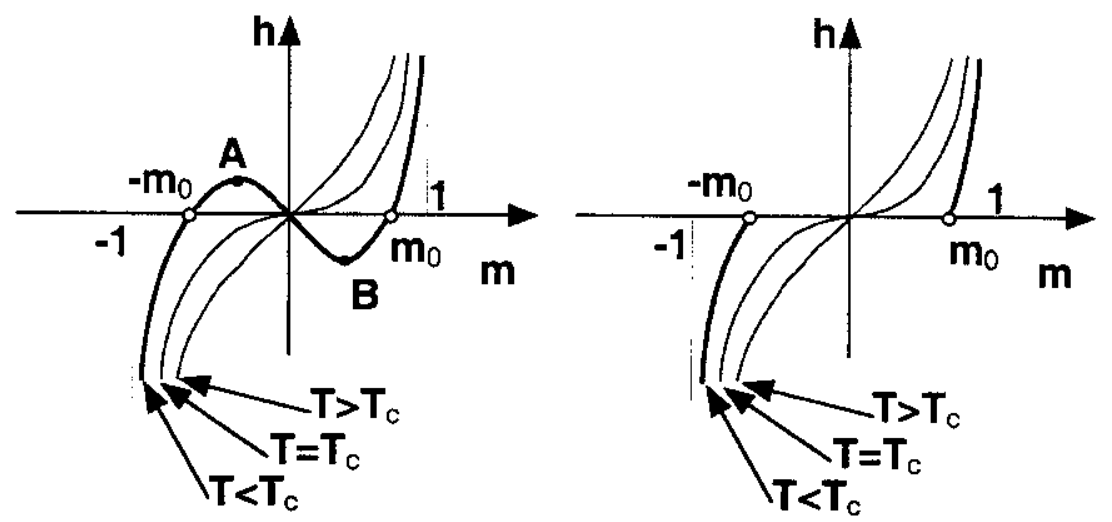

Fig. 2. Left side: relation between the average magnetization and the magnetic field at subcritical, critical and supercritical temperature for the three dimensional Ising model in the mean field approximation. Right side: Maxwell construction modifying the subcritical magnetization curve.

will have the same free energy; such a linear combination represents the coexistence between the two solutions as we have discussed in chapter 2 and corresponds to an horizontal straight line in the $F-m$ and in the $h-m$ plane (tangent construction) as shown in the right part of figure 2 .

If the lack of correlations of the mean field is cured by allowing a mixed phase according to eq. (22), the usual shape of the phase transition is recovered (discontinuity in the first derivative of the thermodynamical potential).

To conclude this section we would like to comment the difference between a self consistent approach as the mean field approximation and a genuine one body Hamiltonian as in eq.(16),(17). We have shown in chapter 1 that the thermodynamics of a system is completely determined once the partition sum is known, since all thermodynamical quantities can be calculated as successive derivatives of $\ln Z$. The Hamiltonian entering the mean field approximation of the partition sum eq.(20) differs from the mean field approximation of the Ising Hamiltonian because of the constant $K$ which we have been forced to add for the Hamiltonian to have the correct expectation value. The constant $K$ in the partition sum represents more than a trivial shift in the energy scale since $K$ depends on $m$ which in turn is calculated from $\ln Z$ showing the self-consistent character of the approach. Following eqs.(16),(17) one could be tempted to define from the mean field approximation to the Ising Hamiltonian a one body partition sum as

$$
Z_{1 b}=\left(\sum_{s= \pm 1} \exp (-\beta(h+J z m) s)\right)^{N}
$$

and the question arises weather thermostatistical observables can be obtained from the successive derivatives of $Z_{1 b}$. 

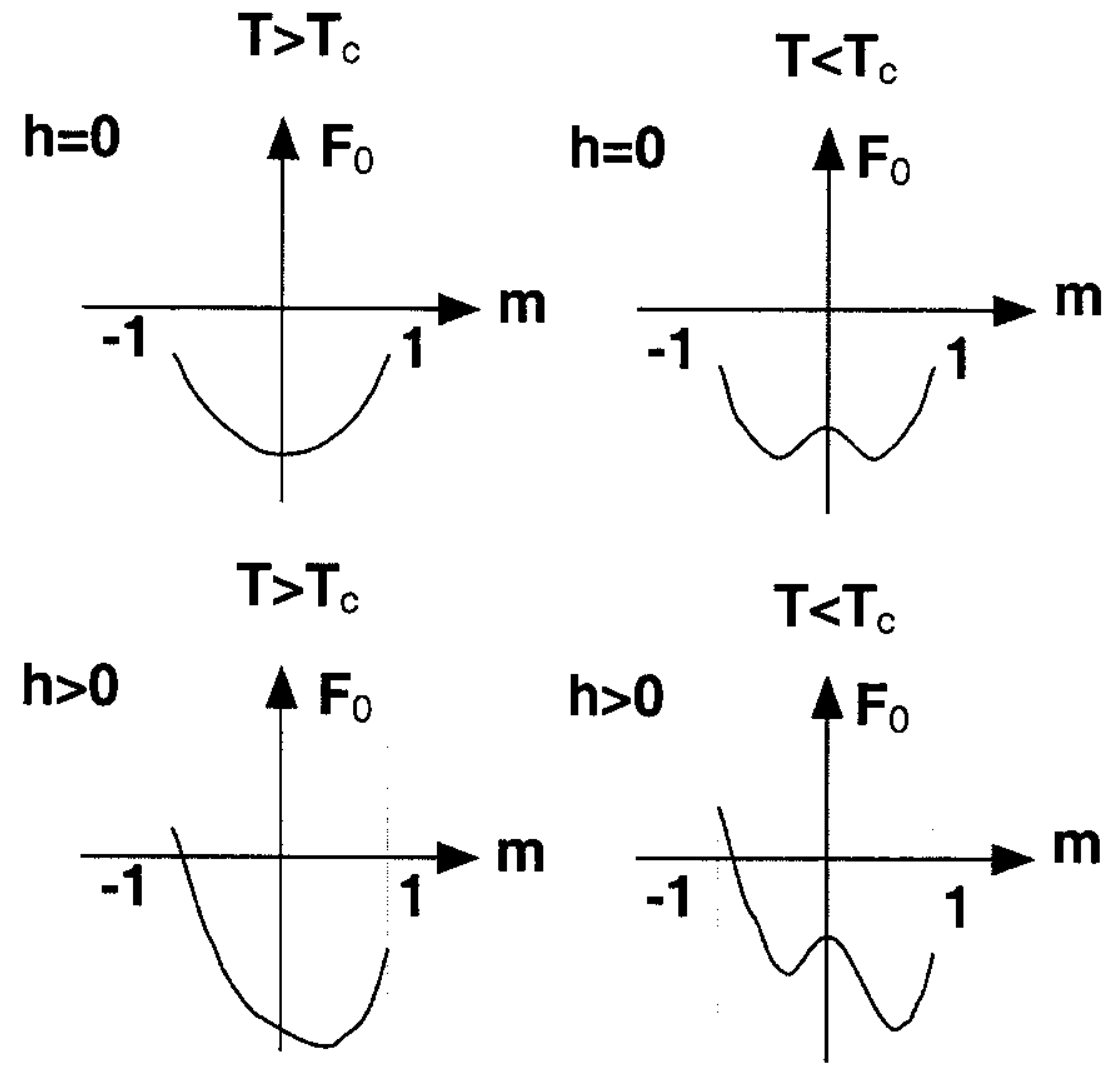

Fig. 3. Mean field free energy as a function of magnetization at zero (upper part) and positive (lower part) magnetic field, for a supercritical (left) and a subcritical (right) temperature.

To answer to this question one has to use the formalism of chapter 1 and explicitly calculate the statistical entropy

$$
\begin{aligned}
S_{M F} & =-\sum_{i} p_{i} \ln p_{i} \\
& =-\sum_{i}\left(p_{i}\left(-\beta H_{1 b}^{(i)}-\ln Z_{1 b}\right)\right) \\
& =\beta\left\langle H_{1 b}\right\rangle+\ln Z_{1 b}
\end{aligned}
$$

since the probability distribution for the mean field problem reads $p_{i}=\exp \left(-\beta H_{1 b}^{(i)}\right) / Z_{1 b}$. The expectation of $H_{1 b}$ is readily calculated as

$$
\left\langle H_{1 b}\right\rangle=-N\left(h m+J z m^{2}\right)=-h N m+2 E_{M F}^{i n t}
$$


The general relation between entropy and free energy $-\beta F \equiv \ln Z_{M F}=$ $S_{M F}-\beta E_{M F}$ finally leads to

$$
\ln Z_{M F}=\ln Z_{1 b}+\beta E_{M F}^{i n t}
$$

Equation (24) shows that because of the two body interaction the partition sum is different from the one body partition sum even in the mean field approximation. In fact the difference comes from the double counting of the two-body interaction if the energy is calculated as $\left\langle H_{1 b}\right\rangle$.

The best way to understand the mean-field approach is to consider mean-field solutions as a trial state to maximize the entropy completed by the constraints $(S-\beta\langle E\rangle)$ i.e. to variationally estimate the free energy $F=-\beta^{-1}(S-\beta\langle E\rangle)$. Then only the mean-field free energy can be considered as a good approximation of the exact free energy leading to $\beta F=-\ln Z_{1 b}-\beta E_{M F}^{\text {int }}$ which is nothing but equation (24).

\subsection{Implications for the liquid-gas transition}

We have seen in section 2.3 that the isomorphism between the Ising model and the Lattice Gas model implies that all physical results concerning magnetic transitions can be easily translated in the fluid language and applied within minor modifications to the liquid-gas transition. To this aim the Ising canonical partition sum $Z^{c a n}$, or free energy $F=-\beta^{-1} \ln Z^{c a n}$, has to be transformed into the Lattice Gas grand-canonical partition sum $Z^{G C}$, or grand potential $\Omega=-\beta^{-1} \ln Z^{G C}$. If we only focus on the interaction part of the Lattice Gas model this leads to

$$
\begin{aligned}
\exp \left(-\beta \Omega_{L G}\right) & =\sum_{n} \exp \left(-\beta\left(H_{L G}^{(n)}-\mu A^{(n)}\right)\right) \\
& =\sum_{n} \exp \left(-\beta\left(H_{I S}^{(n)}-N \frac{z J}{2}-N \frac{\mu}{2}\right)\right) \\
& =\exp \left(-\beta F_{I S}\right) \exp \left(\beta N\left(\frac{z J}{2}+\frac{\mu}{2}\right)\right) .
\end{aligned}
$$

In the mean field approximation $F_{I S}=-\beta^{-1} \ln Z_{M F}$ is given by eq.(20) giving for the Lattice Gas grand potential

$$
\frac{\Omega}{N}=\frac{1}{2} J z m^{2}-\beta^{-1} \ln (2 \operatorname{ch}(\beta(h+J z m)))-\frac{z J}{2}-\frac{\mu}{2}
$$

The total number of lattice sites in the Lattice Gas framework represents the volume of the fluid $N=V$. The equation of state $p=\beta^{-1} \partial_{V} \ln Z$ allows to access the pressure

$$
\begin{aligned}
p & =-\frac{\Omega}{V}=\frac{1}{2}\left(z J+\mu-J z m^{2}\right)+\beta^{-1} \ln (2 c h(\beta(h+J z m))) \\
& =-\varepsilon z \rho^{2}+\beta^{-1} \ln \frac{1}{1-\rho}
\end{aligned}
$$




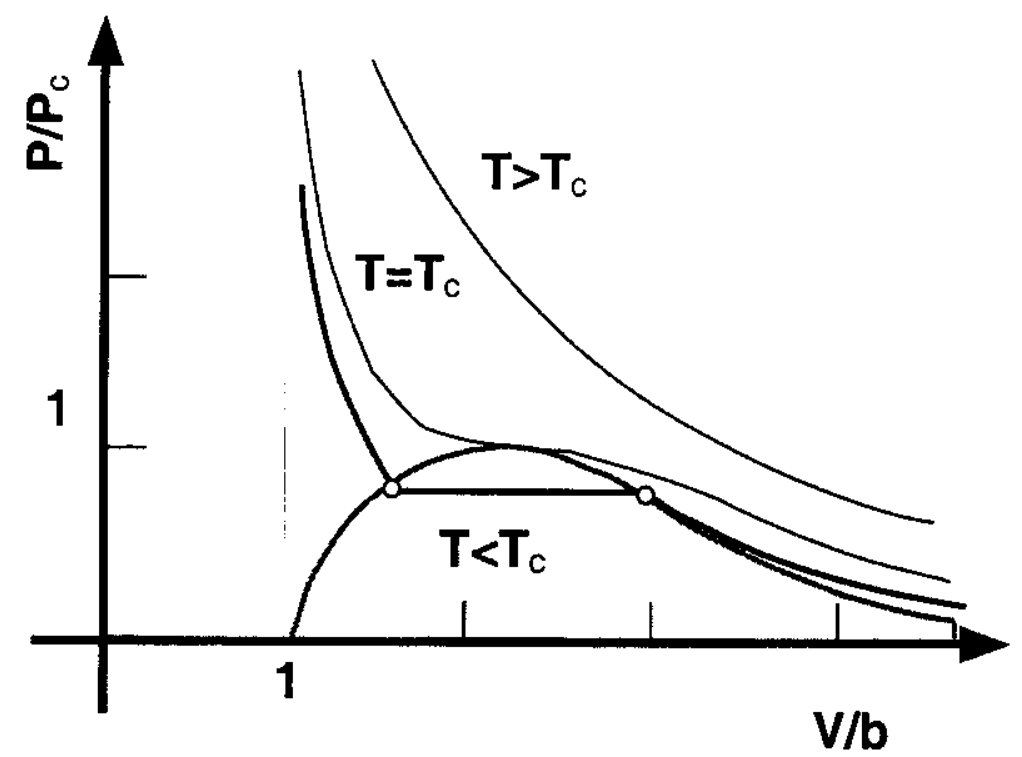

Fig. 4. Isotherms in the pressure versus volume (in cell units $b$ ) plane for the three dimensional Lattice Gas model in the mean field approximation with a Mawell construction of the mixed phase. The coexistence zone is also indicated.

where the last equality is obtained using the magnetization equation of state eq.(21) and the substitutions $m=2 \rho-1, J=\varepsilon / 2$. Figure 4 shows some selected isotherms of the fluid equation of state. At subcritical temperatures $T<T_{c}$ a clear backbending is seen reflecting the instability of the homogeneous mean field solution respect to the separation into distinct phases as in figure 2 above. Once again, if a linear interpolation of the liquid and gas volume solutions is imposed, the usual plateau of the Maxwell construction is recovered. The critical point is defined as the ending point of the coexistence zone, i.e. the point at which the first as well as the second derivative of the equation of state are zero. Substituting in eq. $(25)$ we get $\rho_{c}=1 / 2, T_{c}=J z, p_{c}=T_{c}(\ln 2-1 / 2)$.

\subsection{The Van der Waals equation of state}

The Ising model in the mean field approximation reflects the same physics as the Van der Waals equation of state which describes a classical canonical gas of $N$ identical molecules in an external pressure field $p_{0}$ and volume $V$ interacting via an attractive two body force. The free enthalpy connected to such a physical scenario is

$$
H=F+p_{0} V=\frac{3}{2} N T-b \rho N-T S+p_{0} V
$$



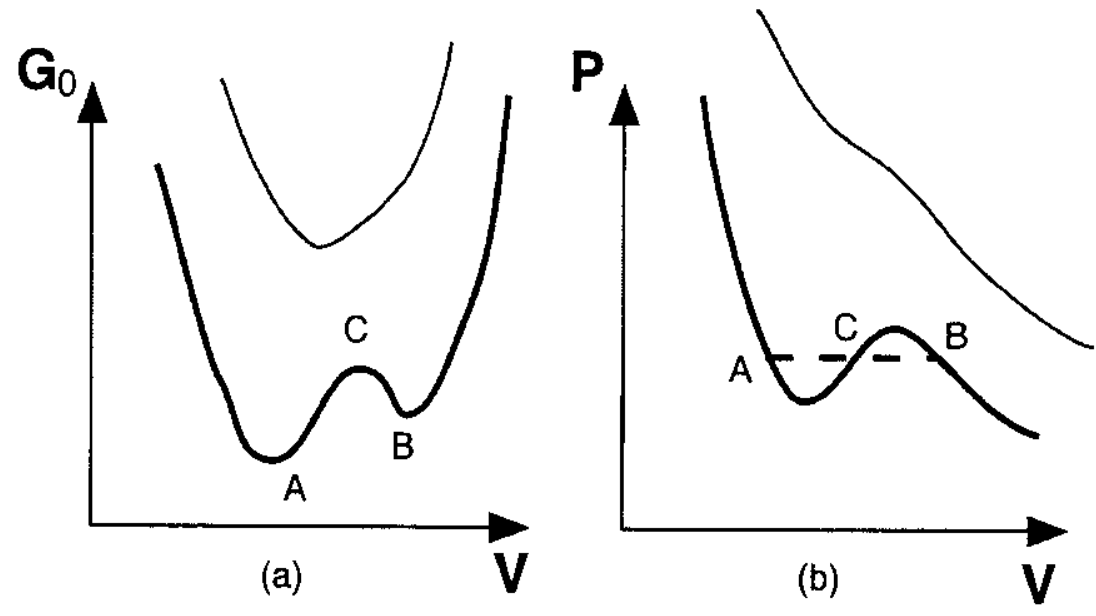

Fig. 5. Free energy and isotherms of the Van der Waals equation of state at a subcritical and supercritical temperature.

Here $\rho=N / V$ is the density of matter and $b \rho N$ is the two body interaction energy in the mean field approximation. If we make an explicit use of the equivalence between ensembles at the macroscopic limit, the entropy $S$ can be calculated in the mean field approximation as an effective one body problem

$$
S=\ln W=\ln \left(W_{r} W_{p}\right)=\ln \left(w_{r}^{N} w_{p}^{N}\right)=N \ln \frac{V-N v_{0}}{N}+\frac{3}{2} N \ln T
$$

where the integral over the configuration space is

$$
w_{r}^{N}=\left(\int_{V} d^{3} r \theta\left(r^{3}-V\right)\right)^{N} / N !=\left(V-N v_{0}\right)^{N} / N !
$$

where $v_{0}$ is the volume occupied by each particle, the momentum space integral gives

$$
w_{p}^{N}=\left(\int d^{3} p \exp \left(-p^{2} / 2 m T\right)\right)^{N}=(2 \pi m T)^{3 N / 2}
$$

and we have used the Stirling approximation of the factorials. Using as above the equation of state $p=\beta^{-1} \partial_{V} \ln Z=-\partial_{V} F$ or the extremum condition $\partial_{V} H=0$ we get

$$
p=\frac{N T}{V-N v_{0}}-\frac{b N^{2}}{V}
$$

The free energy together with the isotherms are represented in figure 5. The similarity with the microscopic results from the Ising model is evident. Once again the volume interval $V_{A}<V<V_{B}$ is unstable in the sense that if we mix up in linear proportions solutions of type $A$ and of type $B$, the free enthalpy 


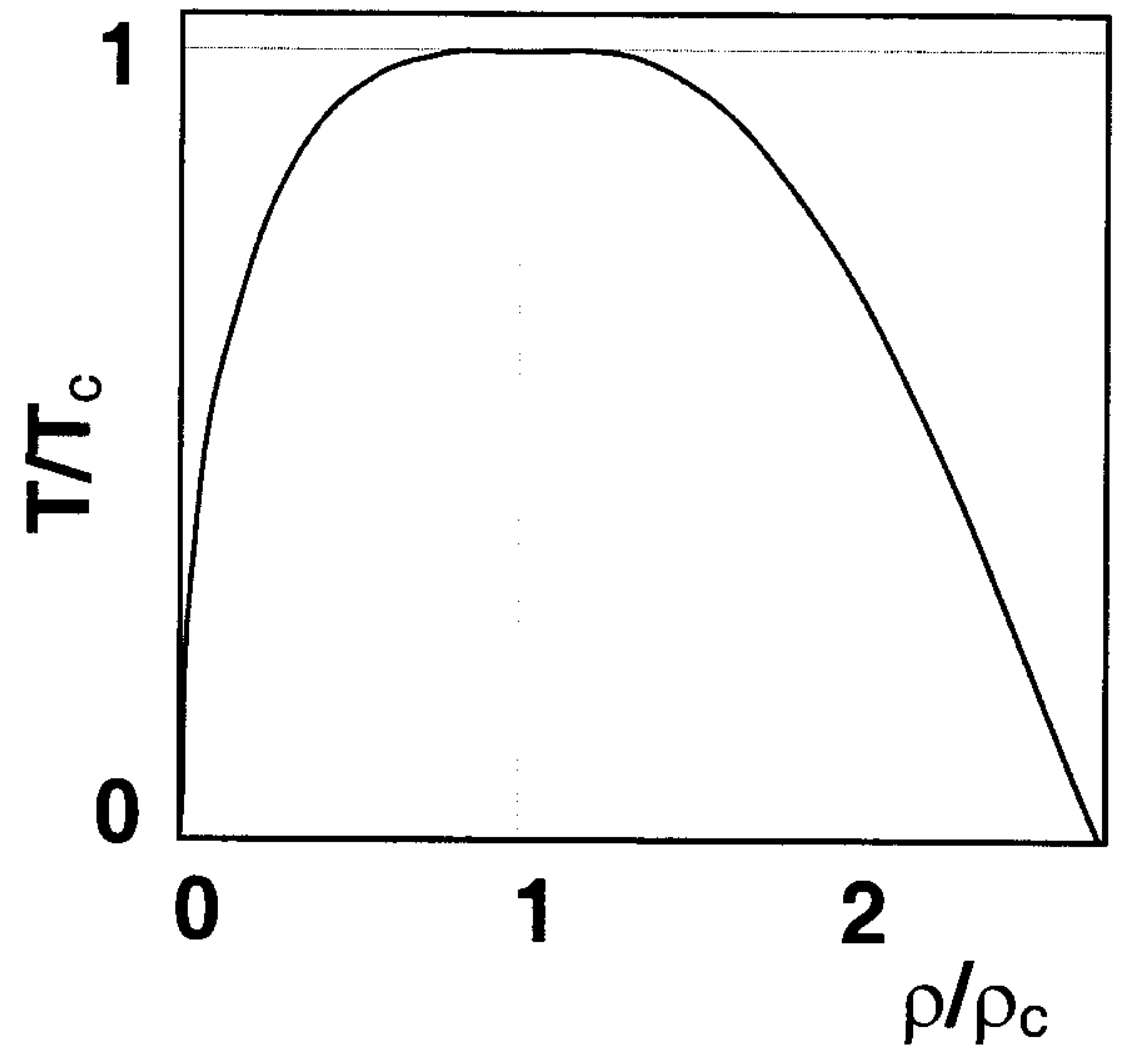

Fig. 6. Guggenheim's coexistence line in scaled variables obtained from many different substances.

$(H)$ does not change. This tangent construction is the well known equal area Maxwell construction since

$$
H\left(V_{B}\right)-H\left(V_{A}\right)=\int_{V_{A}}^{V_{B}} d V d H / d V=p_{0}\left(V_{B}-V_{A}\right)-\int_{V_{A}}^{V_{B}} d V p(V)=0
$$

The coordinates of the critical point are found from $\left.d_{V} p\right|_{c}=\left.d_{V}^{2} p\right|_{c}=0$ as $V_{c}=3 N v_{0}, T_{c}=8 b / 27 v_{0}, p_{c}=b / 27 v_{0}$. If we introduce the scaled variables $v=V / V_{c}, \tau=T / T_{c}, \pi=p / p_{c}$ one can readily verify that the Van der Waals equation (26) becomes

$$
\pi=\frac{8 \tau}{3 \nu-1}-\frac{3}{\nu^{2}}
$$

with no dependence on $b$ or $v_{0}$ i.e. on the quantities specific of the structure of the gas.

This feature is preserved in realistic gases for which all thermodynamical variables can be rescaled according to the critical values leading to the famous Guggenheim phase diagram [9] (shown in figure 6), namely a unique coexistence 
curve for many different substances which shows the universality character of phase transitions; this universality feature gives an a posteriori justification of the use of a schematic oversimplified model as the Ising model to describe complex and widely different physical phenomena.

\subsection{The Landau Model}

The simplest functional form of the thermodynamical potential as a function of the order parameter that contains all the physical situations discussed in the previous sections is given by

$$
F(m)=C+N\left(a(T) m^{2}+b m^{4}-h m\right) \quad ; \quad a(T)=a_{0}\left(T-T_{c}\right)
$$

where $h$ is the intensive variable conjugated to the order parameter $m$. Note $b>0$ in order to have a free energy bound from below in order to ensure that an absolute minimum (i.e. an equilibrium) does exist. Equation 27 is known in the literature as the result of Landau theory of phase transitions[10]. It is immediate to verify that in the proximity of the critical point $T \rightarrow T_{c}, h \rightarrow 0$ the order parameter and its derivatives follow a power law behavior $m \rightarrow\left|T_{c}-T\right|^{\beta}$, $d m / d h \rightarrow\left|T_{c}-T\right|^{\gamma}$ where $\beta=1 / 2, \gamma=-1$ are typical mean-field critical exponents.

Summarizing the present chapter, the mean field approximation leads to the definition of phase transitions as universal phenomena with the following characteristics

- presence of two different phases (i.e. minima of the thermodynamical potential) that coexist in contact (via a non analyticity or tangent construction that mixes the two solutions in linear proportions)

- existence of critical points (or second order phase transitions) that correspond to the limit of the coexistence line

- definition of an order parameter (i.e. the extensive observable that allows to distinguish the two phases) that presents a discontinuity at the (first order) phase transition.

\section{Finite systems: getting more from pushing less}

In the preceding sections we have defined a first order phase transition as a discontinuity in the first derivative (or order parameter) $m$ of the thermodynamical potential $F$ as a function of the control parameter. Such a discontinuity can exist only in the thermodynamical limit since

- this discontinuity corresponds to phase coexistence according to the equation (see section 2.2)

$$
F\left(\alpha m_{1}+(1-\alpha) m_{2}\right)=\alpha F\left(m_{1}\right)+(1-\alpha) F\left(m_{2}\right)
$$

which holds if the free energy per particle is independent of the number of particles, i.e. if surface can be neglected respect to volume which is only possible if the volume goes to infinity. 
- if the system is finite the partition sum is a sum over a finite number of configurations, i.e. an analytic function. As a consequence, its first order derivative $\langle m\rangle$ according to the corresponding equation of state cannot present discontinuities.

For these reasons, it is often stated that phase transitions are only defined for infinite systems. Following this viewpoint, finite systems can present only smooth phase changes (cross-over); to demonstrate the asymptotic existence of a phase transition a careful study of the behavior of thermostatistical variables with the size of the system (finite size scaling[11]) has to be performed; in this context, finite size scaling is also the only way to determine the order of a transition if one believes that in finite systems all transitions are smooth

These considerations are based on analyses where the intensive variable associated with the order parameter is controlled (i.e. the order parameter is only measured in average). In such a case, indeed the equation of state are always smooth in finite system. But what happens in other statistical ensembles?

A fundamental theorem in statistical mechanics, the Van Hove theorem, demonstrated in the next session, guarantees the equivalence between different statistical ensembles at the thermodynamical limit. (However, it tells nothing about finite systems except that the theorem cannot be demonstrated). However, if this equivalence between statistical ensembles is a good approximation even for finite systems, an experiment where the order parameter is controlled (e.g. for the Ising model all events in the statistical sample share the same value of the magnetization) will lead to the same equation of state as the one obtained when the conjugated field is imposed to the system but the magnetization remains free to fluctuate. Then the sudden jump of the magnetization observed in infinite systems will be replaced by a smooth variation. If this would be true, the existence of the transition in the finite system could only be proved through finite size scaling techniques.

This is in fact not the case.

In the next sections we will show that the Van Hove theorem is violated in first order phase transitions if the system is finite, and this violation can persist up to the thermodynamical limit in the case of long range forces. A consequence of that is that it will be possible to give a rigorous definition of phase transitions even in finite systems, with the prediction (and in some case the experimental evidence, see chapters 7,8 ) of fancy phenomena as negative heat capacities, negative compressibilities and negative susceptibilities.

The non-equivalence of statistical ensembles has also important conceptual consequences. It implies that the value of thermodynamical variables for the very same system depends on the type of experiment which is performed (i.e. on the ensemble of constraints which are put on the system), contrary to the standard thermodynamical viewpoint that water heated in a kettle is the same as water put in an oven at the same temperature. This point will be discussed in chapter 6. 

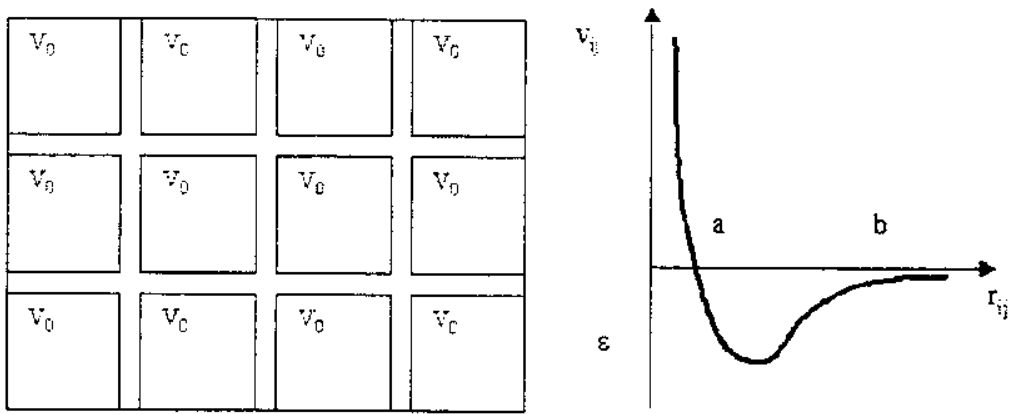

Fig. 7. Schematic representation of the Van Hove theorem demonstration (left) and the corresponding interparticle interaction

\subsection{The Van Hove theorem}

Let us consider a system in a volume $V$ for which only the average value of energy and number of particles is defined (grancanonical ensemble). Let us divide $V=m V_{0}+V_{1}$ in $m$ equal boxes separated by a "corridor" of width $b$ larger than the range of the force such that the interactions among particles in different boxes can be neglected, (see figure 7). Let us calculate the grand potential $\Omega=-T \ln Z$

$$
Z_{\beta \mu}(V)=\sum_{n} \exp \left(-\beta\left(H^{(n)}-\mu N^{(n)}\right)\right)
$$

where the sum extends over all the possible configurations of the system, $H^{(n)}=$ $K^{(n)}+U^{(n)}\left(N^{(n)}\right)$ represents the energy (number of particles) of the system in the configuration $(n)$, and $\beta, \mu$ are the associated Lagrange multipliers, the inverse temperature and the chemical potential respectively. The partition sum results

$$
\begin{aligned}
& Z_{\beta \mu}(V)=\sum_{N=0}^{\infty} \frac{1}{N !} \frac{1}{h^{3 N}} \int_{V} d^{3 N} r \int d^{3 N} p \exp (-\beta(H-\mu N)) \\
& Z_{\beta \mu}(V)=\sum_{N=0}^{\infty} z_{k}^{N} \frac{1}{N !} \int_{V} d^{3 N} r \exp (-\beta U)=\sum_{N=0}^{\infty} z_{k}^{N} Z_{\beta}(N, V)
\end{aligned}
$$

with $z_{k}=\exp (\beta \mu)\left(\frac{2 m \pi}{h^{2} \beta}\right)^{3 / 2}$ and $Z_{\beta}(N, V)=\frac{1}{N !} \int_{V} d^{3 N} r \exp (-\beta U)$. This represents the well known relation between the grancanonical partition sum $Z_{\beta \mu}(V)$ and the interaction part of the canonical one $Z_{\beta}(N, V)$. To calculate $Z_{\beta}(N, V)$ let us consider introduce the number of particles in the corridor $N_{1}$

$$
Z_{\beta}(N, V)=\sum_{N_{1}=0}^{N} \frac{1}{N_{\perp} !} \frac{1}{\left(N-N_{1}\right) !} \int_{V_{1}} d^{3 N_{1}} r \int_{V-V_{1}} d^{3\left(N-N_{1}\right)} r \exp (-\beta U)
$$


Let us note $\epsilon$ the minimum of the two body interaction (see figure 7); the potential energy in the corridor satisfies then the inequality $U_{V_{1}} \geq \varepsilon \xi N_{1}$, where $\xi=(b / a)^{3}$ represents the maximum number of particles interacting with a given particle. For the total potential energy we can then write

$$
U \geq \varepsilon \xi N_{1}+\frac{1}{2} \sum_{i=N_{1}+1}^{N} \sum_{j=N_{1}+1}^{N} v_{i j}
$$

which implies for the partition sum

$$
\begin{aligned}
Z_{\beta \mu}(V) & \leq \sum_{N_{1}=0}^{\infty} \frac{1}{N_{1} !} V_{1}^{N_{1}} z_{k}^{N_{1}} \exp \left(-N_{1} \beta \varepsilon \xi\right) \sum_{N_{2}=0}^{\infty} \frac{1}{N_{2} !} z_{k}^{N_{2}} \int_{n V_{0}} d^{3 N_{2}} r \exp (-\beta U) \\
& =\exp \left(z_{k} V_{1} e^{-\beta \varepsilon \xi}\right) Z_{\beta \mu}^{m}\left(V_{0}\right)
\end{aligned}
$$

where the last equality stems from the fact that particles interact only within the same box again because of the short range of the force. Finally we get using $V_{1} \propto m V_{0}^{2 / 3}$

$$
\begin{aligned}
\ln Z_{\beta \mu}(V) \leq & k m V_{0}^{2 / 3}+m \ln Z_{\beta \mu}\left(V_{0}\right) \\
& \frac{\ln Z_{\beta \mu}(V)}{V} \leq k V_{0}^{-1 / 3}+\frac{\ln Z_{\beta \mu}\left(V_{0}\right)}{V_{0}}
\end{aligned}
$$

which gives in the thermodynamical limit (keeping $m$ constant) $V \rightarrow \infty, V_{0} \rightarrow$ $\infty, V \rightarrow m V_{0}$

$$
\frac{\ln Z_{\beta \mu}(V)}{V} \leq \frac{\ln Z_{\beta \mu}\left(V_{0}\right)}{V_{0}}
$$

On the other side the opposite inequality is trivially true

$$
Z_{\beta \mu}(V) \geq Z_{\beta \mu}^{m}\left(V_{0}\right)
$$

since by neglecting the corridor in the integral (28) a positive term in the partition sum is neglected. In conclusion we have demonstrated that

$$
\frac{\ln Z_{\beta \mu}(V)}{V} \underset{V \rightarrow \infty}{\longrightarrow} \frac{\ln Z_{\beta \mu}\left(V_{0}\right)}{V_{0}}
$$

It is very important to stress that this result is true only in the thermodynamical limit and for short range interactions. For these specific systems the implications of eq.(29) can be summarized as follow

- A thermodynamical limit exists for these systems. Indeed if the thermodynamical potential per unit volume tends to a constant independent of the volume (or the number of particles) $\ln Z_{\beta \mu}(V) / V \rightarrow \omega$ the observables distributions $P_{N}(A)$ will also tend to an asymptotic function

$$
\lim _{N \rightarrow \infty} P_{N}(A)=\tilde{p}\left(\frac{A}{N}\right)^{N}
$$



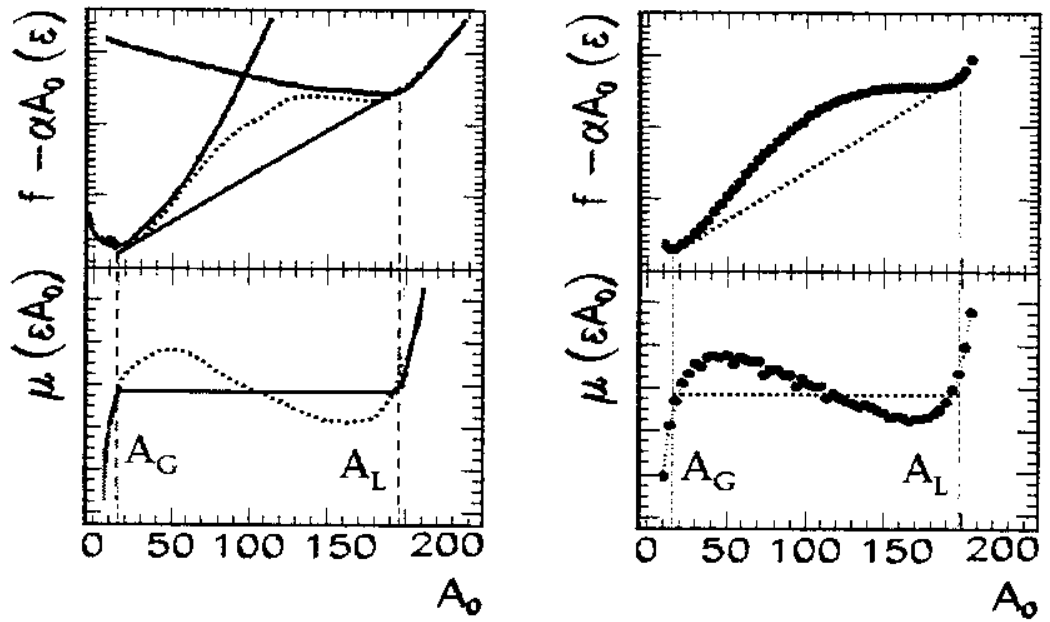

Fig. 8. Schematic representation of the liquid-gas phase transition in the canonical ensemble for an infinite system (left side) and a finite system (right side).

- In the thermodynamical limit ensembles are equivalent. Indeed if $\omega=$ $Z_{\beta \mu}\left(V_{i}\right) / V_{i}$ for an arbitrary subsystem $V_{i}$ this implies that reduced extensive variables are intensive, i.e. that the asymptotic distribution $\widetilde{p}\left(\frac{A}{N}\right)^{N}$ has a vanishing width in agreement with the central limit theorem section 2.2 . Since ensembles differ at the level of fluctuations, this demonstrates the equivalence between ensembles.

In the next section we will show that the violation of the Van Hove theorem in finite systems leads to the emergence of new thermodynamical phenomena in first order phase transitions of mesoscopic systems.

\subsection{Convexity anomalies and phase transitions}

Let us consider the thermodynamics of a first order phase transition in an extensive ensemble, i.e. in the physical case where the order parameter is a controlled variable. A schematic representation of the liquid gas phase transition in the canonical ensemble is given in the left part of figure 8 . The mean field solution for the free energy (dotted line) $F\left(A_{0}, V\right)$ at a given temperature shows two minima in the spirit of Landau theory, corresponding to a gas-like solution at low density (light grey) and a liquid-like solution at high density (dark grey). A tangent construction (straight line) corresponds to the inclusion in the partition sum of mixed partitions given by linear combinations of the two solutions; this linear interpolation is only possible in infinite systems for which we can neglect the role of the interface (the "corridor" between the two phases); this leads to a plateau in the conjugated intensive variable $\mu=\partial F / \partial A_{0}$. If the system is finite (right part) the free energy per particle of the liquid fraction is higher than in 


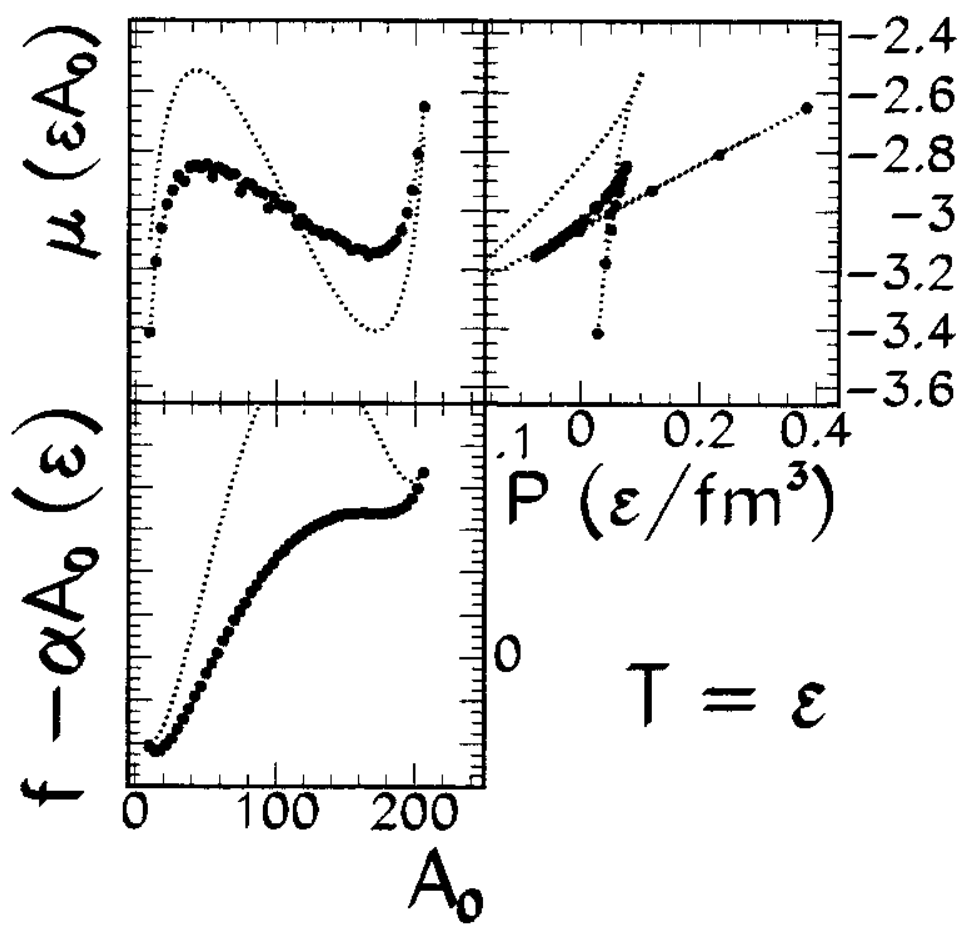

Fig. 9. Free energy, chemical potential and pressure for a $6 \times 6 \times 6$ Lattice Gas at a subcritical temperature. Dashed lines: mean field approximation; symbols: exact results.

the case of the pure liquid solution because of the increased surface tension; as a result the free energy of the mixed configuration is higher than the tangent construction, i.e. is a concave function of $A_{0}$, giving rise to a backbending in the chemical potential and a negative susceptibility $\chi^{-1}=\partial_{A_{0}} \mu$.

This is illustrated in figures 9,10 that show the free energy, chemical potential and pressure obtained from a canonical Monte Carlo simulation of the lattice gas model in a cubic box of linear dimension $L=6$ for different values of the temperature [12], [13]. The convexity anomaly of the partition sum is in the particle density $\rho=A_{0} / L^{3}$ direction which represents the order parameter of the transition; $\rho$ can be seen both as a number of particles at fixed volume or as an inverse number of lattice sites at fixed number of particles. For this reason the same anomalous backbending presented by the chemical potential is observed in the $p(V)$ equation of state as shown in figure 11. All isotherms up to the critical temperature backbend, i.e. show a region of negative compressibility.

In figs. 9, 10, 11 the dashed lines represent the mean field approximation discussed in chapter 3 . Not surprisingly, the mean field badly fails in the phase tran- 


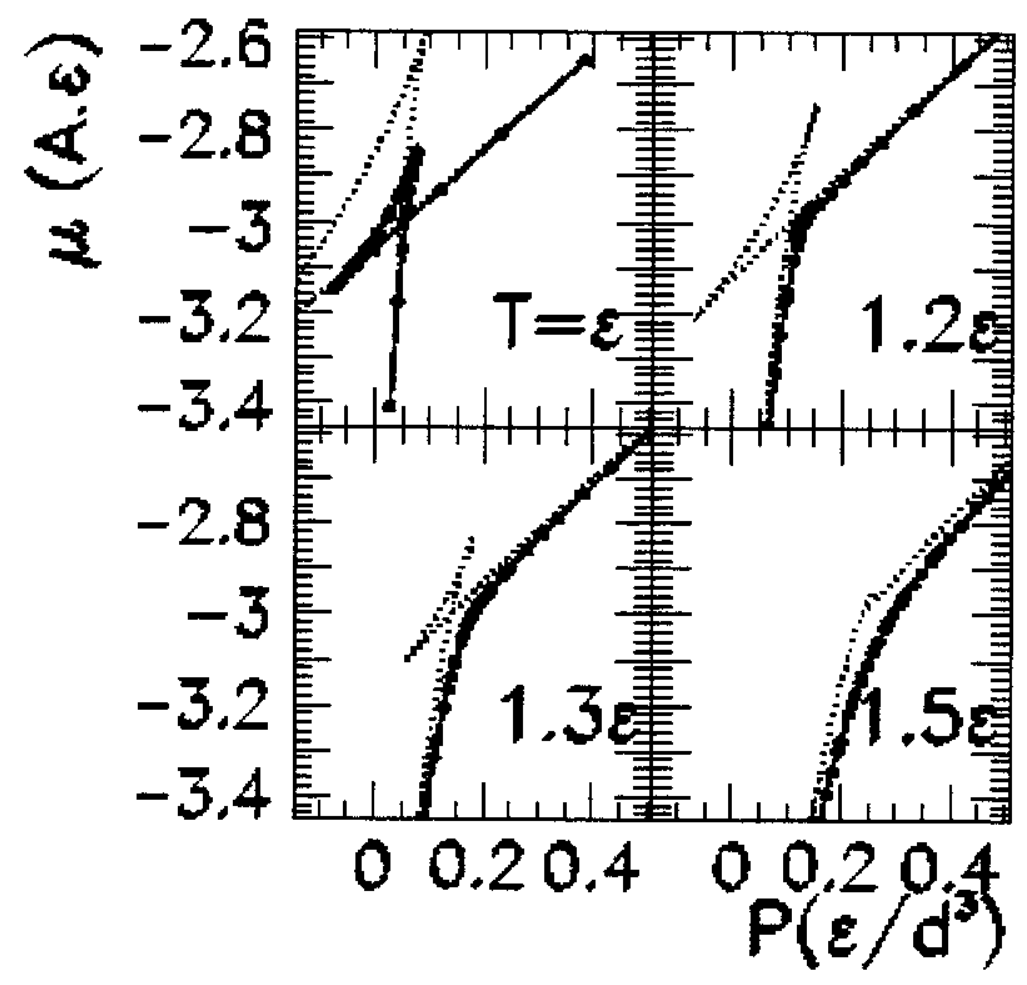

Fig. 10. Chemical potential as a function of pressure at different temperatures for a 6x6x6 Lattice Gas. Dashed lines: mean field approximation; symbols: exact results.

sition region while it is close to the exact result for supercritical temperatures. In particular the Van der Waals loop is still present (see fig.10) at temperatures at which the two phases have merged into one in the exact calculation. This means that the critical temperature is overestimated by the mean field approximation because of its intrinsic lack of fluctuations.

It is important to stress that the physical origin of the backbendings and loops is completely different in the exact calculation and in the mean field. In the former the system inside coexistence presents inhomogeneous partitions that are stable equilibrium solutions with negative compressibility and susceptibility, while in the latter the backbending reflects the instability of the homogeneous mean field solutions respect to phase separation (tangent constructions).

The same reasoning as in figure 8 can be done for the microcanonical ensemble as schematically shown in fig.12[14],,[15]. If energy is controlled, the appropriate thermostatistical potential is the microcanonical entropy. A first order phase transition can be viewed as the sudden opening of a new disordered phase at a certain threshold energy. The number of states of the disordered phase 


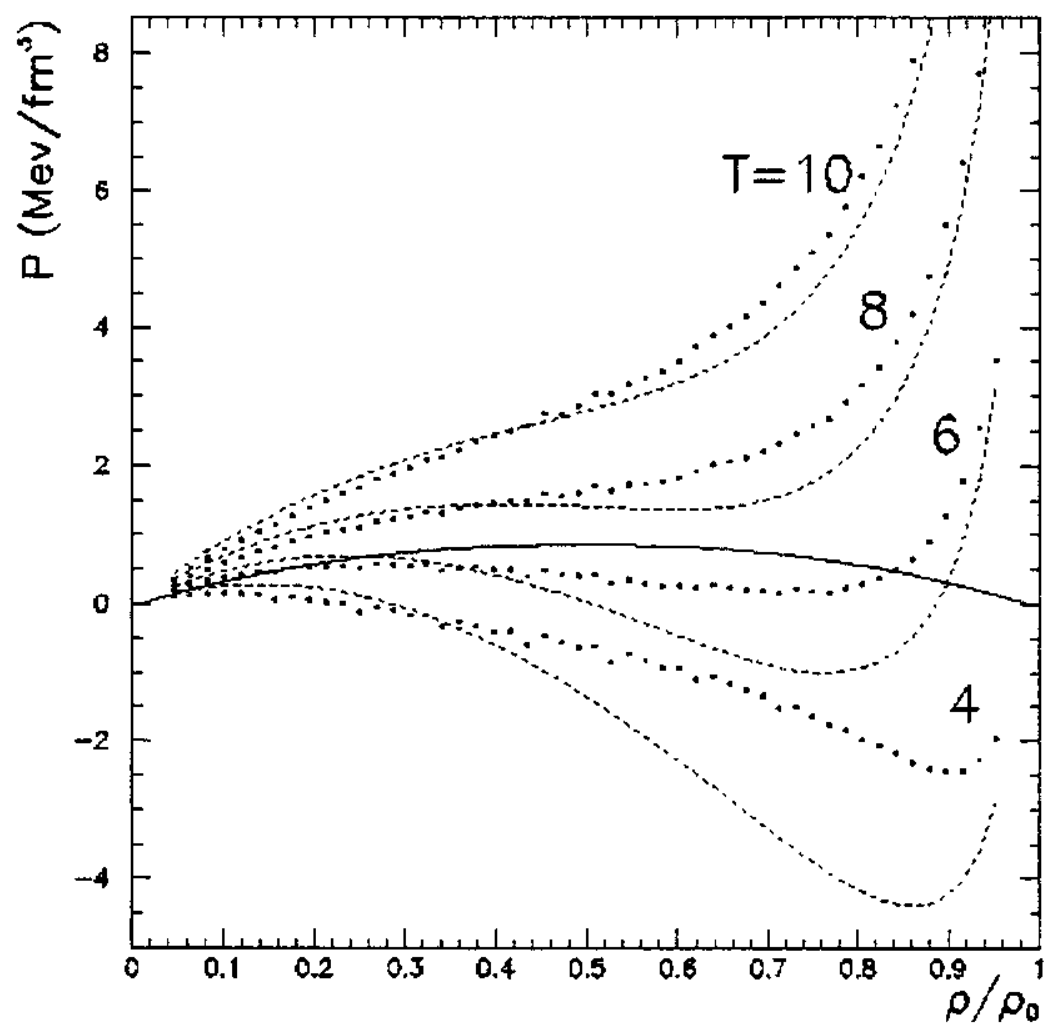

Fig. 11. Pressure versus density equations of state for a $6 \times 6 \times 6$ Lattice gas exactly (symbols) and in the mean field approximation (dashed lines). Solid line: coexistence curve.

grows much faster with energy than the one associated to the ordered phase, and this creates a convex intruder in the total microcanonical entropy. This convexity anomaly cannot be cured by a conventional tangent construction because of the non negligible surface entropy at the interface between the two phases. This phenomenon is in fact observed in a Monte Carlo simulation of the Lattice Gas model in the isobar microcanonical ensemble as shown in fig.13[16], [17]. The convex intruder implies a backbending in the temperature $T^{-1}=\partial_{E} S$ and a negative branch for the heat capacity $C^{-1}=\partial_{E} T$ between two divergences. Density being the order parameter of the liquid gas phase transition, since the number of particles is fixed in this calculation $\left(A_{0}=216\right)$ the volume has to increase with energy to allow the system explore the partitions belonging to the disordered phase. To this aim, the calculation shown in fig.13 are performed at constant pressure, where pressure is here defined as the Lagrange multiplier conjugated to the system volume. In this calculation the system volume is assumed to be the average cubic radius. The corresponding caloric curves at constant pressure and 


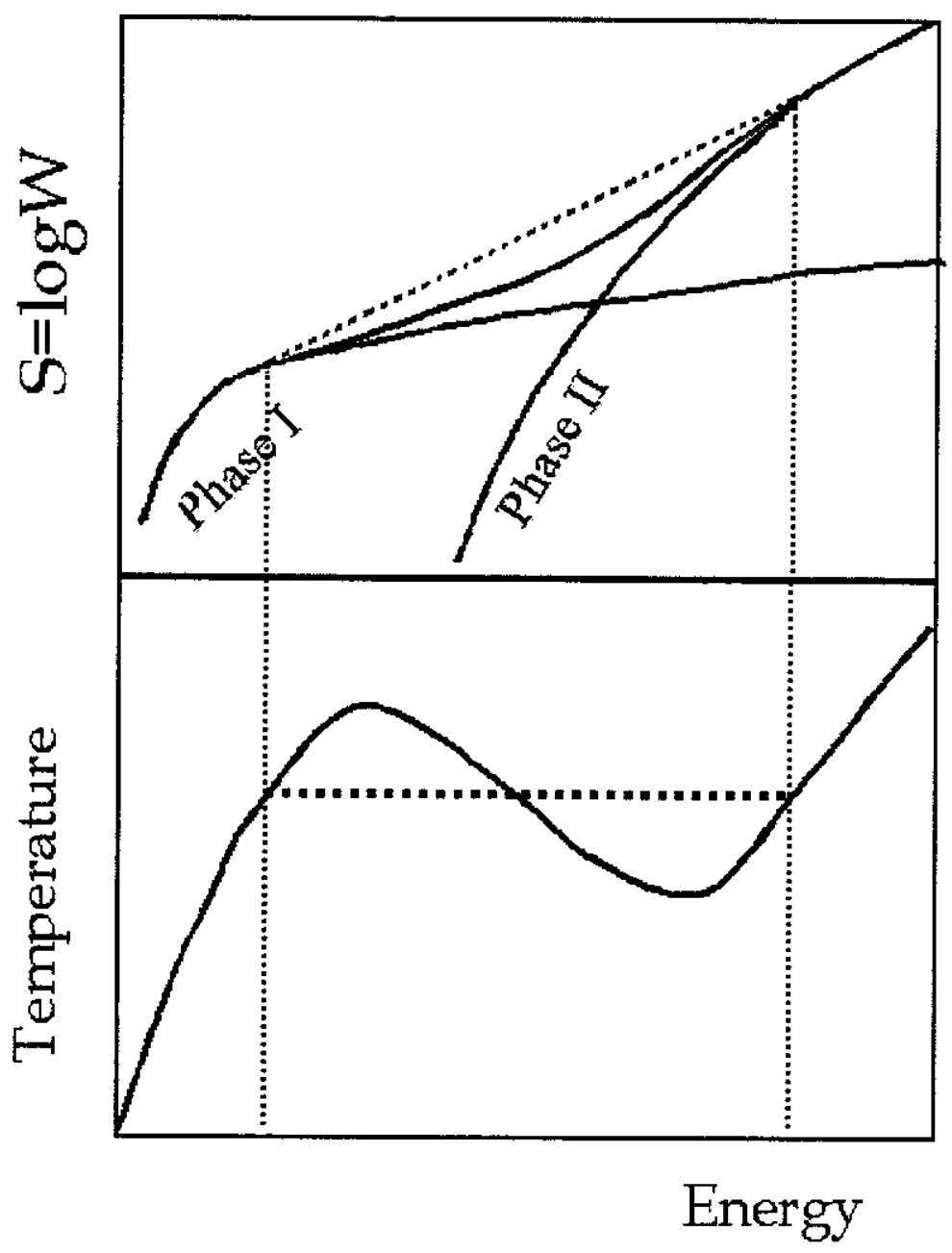

Fig. 12. Schematic representation of a first order phase transition in the microcanonical ensemble.

constant volume are shown in figure 14 . The isobaric curves show a backbending up to the critical pressure giving a clear definition of the coexistence zone.

To summarize, we have shown through some selected examples that a first order phase transition in a finite system is associated to a convexity anomaly in the appropriate thermostatistical potential; the direction of the anomaly in the space of observables can be defined as the order parameter of the transition and the conjugated intensive variable shows a backbending in the coexistence zone; energetic considerations suggest that this backbending transforms into a 


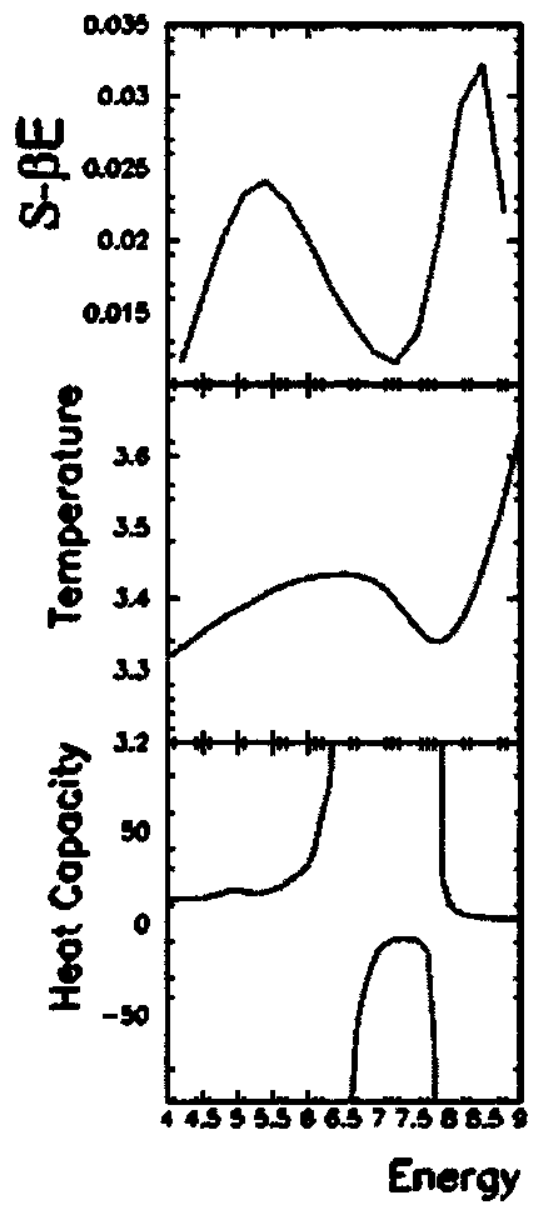

Fig. 13. Entropy, temperature and heat capacity for 216 particles in the microcanonical Lattice Gas model at a subcritical pressure.

plateau (standard first order phase transition) if surfaces are negligible in the global energetic of the infinite system (c.f.. Van Hove theorem).

In particular in the microcanonical ensemble adding energy to the system can cause its temperature to decrease and we have intuitively associated the observation of negative heat capacity with the sudden opening of a disordered collective channel[18]. To clarify the link between channel openings, phase transitions and negative heat capacities in the next sections we will consider some simple analytical equilibrium models that exhibit negative heat capacity. 


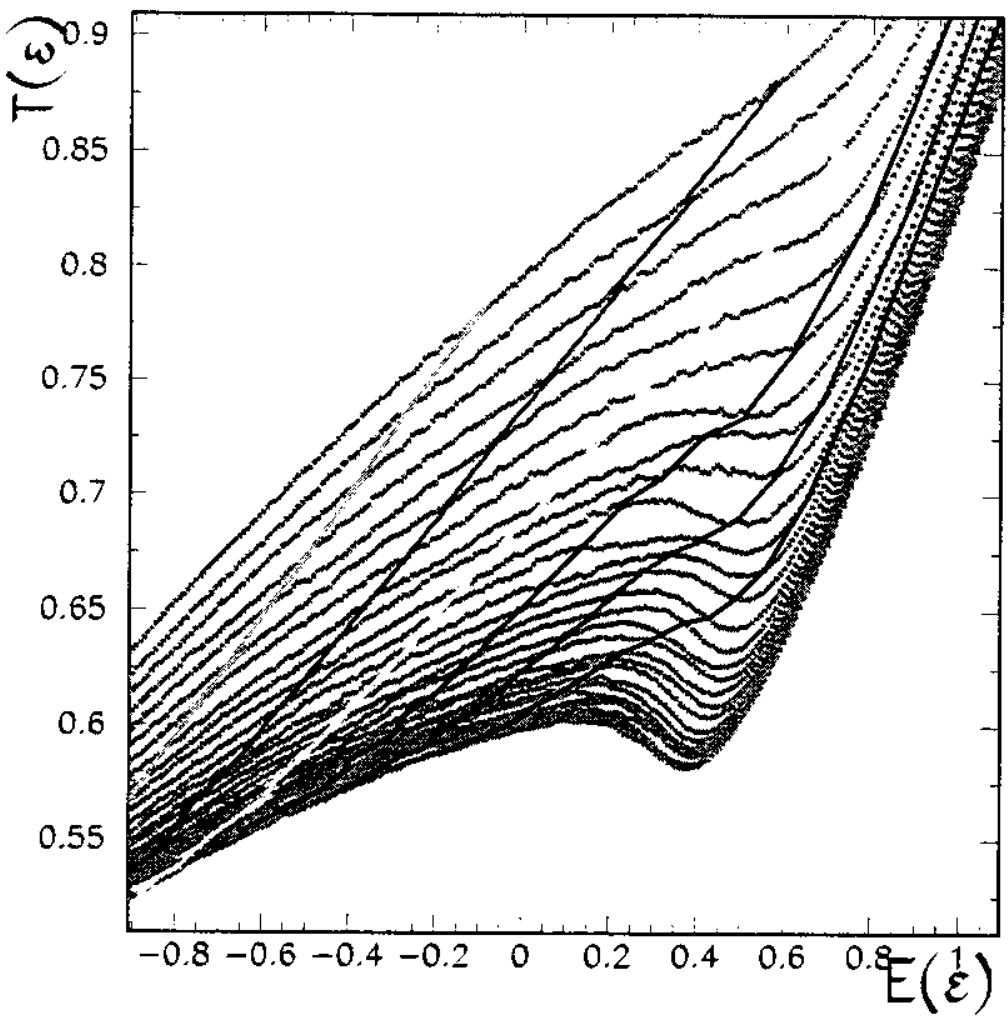

Fig. 14. Caloric curves at constant pressure (showing a backbending up to the critical pressure) and at constant volume (monotonically increasing) for 216 particles in the microcanonical Lattice Gas model.

\subsection{Interacting particles in harmonic potentials: the classical case}

To see the link between the opening of a channel and negative heat capacity let us consider the simple example of $A$ classical particles in two harmonic oscillators of different frequency $\omega_{1}, \omega_{2}$ such that the particles in the first oscillator interact all with a constant coupling $\varepsilon$, while they are free in the second one. This model can be seen as a schematic representation of the liquid-gas phase transition at constant pressure. The Hamiltonian reads

$$
H=\sum_{i=1,2} \sum_{k=1}^{A_{i}} \frac{p_{k}^{2}}{2 m}+\frac{1}{2} k_{i} x_{k}^{2}+\varepsilon A_{1}^{2}
$$

where $A_{i}$ is the number of particles in the $i$-th oscillator. The entropy of such a system is exactly calculable. Indeed the number of states with energy $e \leq E$ 
accessible to a particle in an harmonic oscillator with frequency $\omega=\sqrt{k / m}$ is

$$
\Omega(E)=\int_{\sigma(E)} \frac{d x d p}{\hbar}=\frac{2 \pi E}{\hbar \omega}
$$

where $\sigma(E)$ is the surface in phase space defined by the iso-energy curve $p^{2} / 2 m+$ $k x^{2} / 2=E$. Similarly the number of states with energy $e \leq E$ accessible to $A$ particles will be given by

$$
\Omega_{A}(E)=\left(\frac{2}{\hbar \omega}\right)^{A} V_{2 A}(\sqrt{E})
$$

where $V_{n}(x)$ is the volume of an hypersphere in $n$ dimensions with radius $x$. The state density $W(E)=d \Omega / d E$ results

$$
W_{A}^{\omega}(E)=\left(\frac{2 \pi}{\hbar \omega}\right)^{A} \frac{E^{A-1}}{(A-1) !}
$$

This formula can be generalized to the case of $A_{1}\left(A_{2}\right)$ particles in the oscillator at frequency $\omega_{1}\left(\omega_{2}\right)$ such that the total sum gives $A$, i.e. the case $(30)$ with no interaction, $\varepsilon=0$ :

$$
\begin{aligned}
& \Omega_{A}(E)=\sum_{A_{1}=0}^{A} \frac{A !}{A_{1} !\left(A-A_{1}\right) !}\left(\frac{2}{\hbar \omega_{1}}\right)^{A_{1}}\left(\frac{2}{\hbar \omega_{2}}\right)^{A-A_{1}} V_{2 A}(\sqrt{E}) \\
& W_{A}(E)=\left(\frac{2 \pi}{\hbar}\right)^{A} \frac{E^{A-1}}{(A-1) !}\left(\frac{1}{\omega_{1}}+\frac{1}{\omega_{2}}\right)^{A}
\end{aligned}
$$

which is equivalent to a unique oscillator at intermediate frequency, $\omega^{-1}=$ $\omega_{1}^{-1}+\omega_{2}^{-1}$. The entropy of this model $S(E) \propto \ln (E)$ is a regular function of positive concavity. Eq.(31) shows that the possible existence of the system in two configurations of different density does not imply a phase transition. However if we introduce an interaction in the first oscillator $(\varepsilon \neq 0)$ the situation drastically changes. Indeed in this case to have a total energy $E$, the energy of the $A_{1}+A_{2}$ independent particles that enters the hypersphere volume has to be $E-\varepsilon A_{1}^{2}$ and the sum in eq. (31) becomes

$$
W_{A}^{\varepsilon}(E)=\sum_{A_{1}=0}^{A} \frac{A !}{A_{1} !\left(A-A_{1}\right) !}\left(\frac{\omega_{1}}{\omega_{2}}\right)^{A-A_{1}} W_{A}^{\omega_{1}}\left(E-\varepsilon A_{1}^{2}\right)
$$

If the second oscillator is sufficiently soft the microcanonical entropy presents a convex intruder leading to a backbending of the caloric curve and negative heat capacity as shown in figure 15. The same anomaly observed in the density of states is present in the average kinetic energy (dashed line in figure 15) showing that the convex intruder corresponds indeed to a physical cooling of the system (see section 4.6 for a deeper discussion on this subject). 


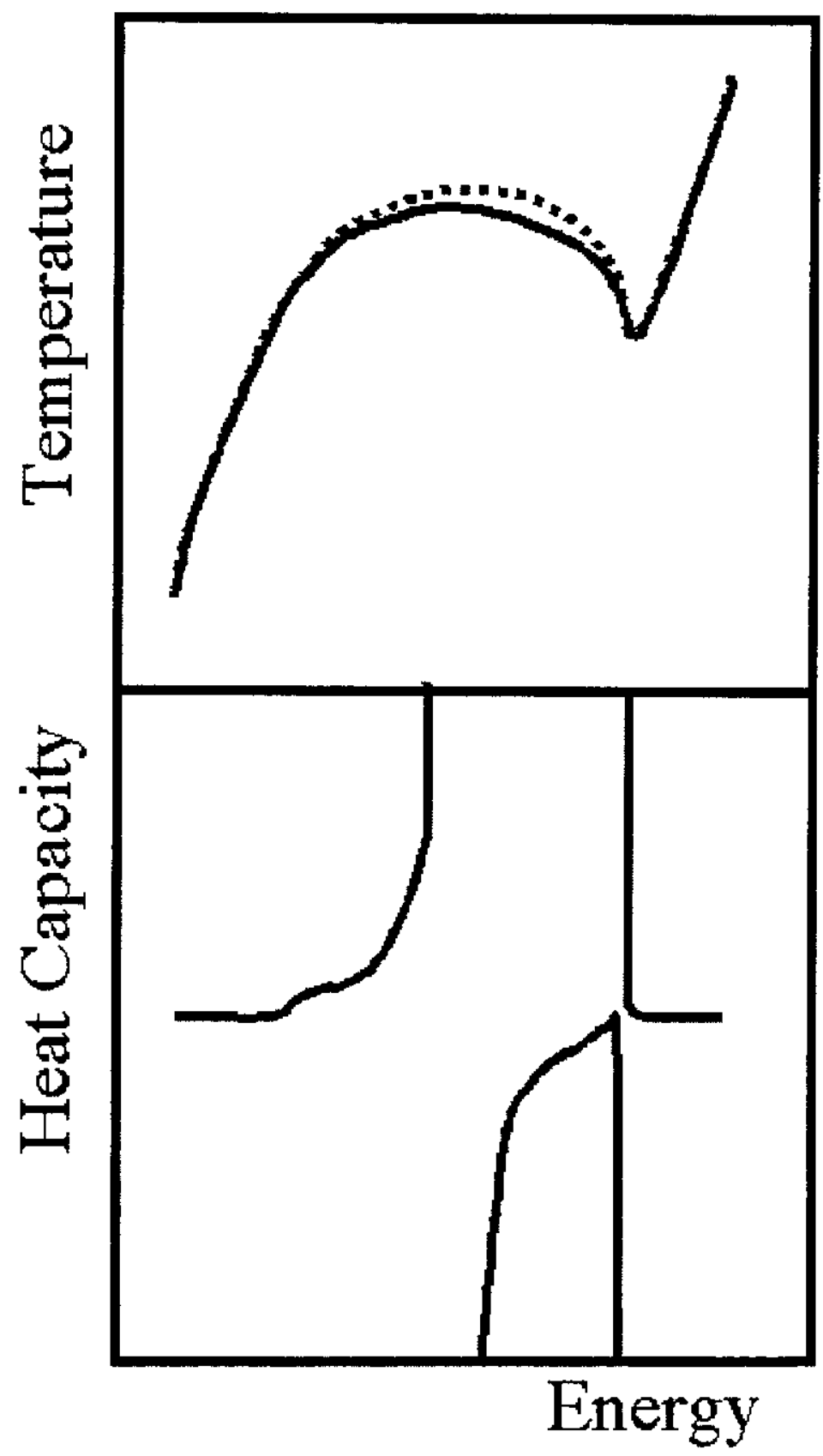

Fig. 15. Temperature and heat capacity for the classical two oscillator model in the subcritical regime. Full curves: derivatives of the entropy. Dashed curve: average kinetic energy per degree of freedom. 


\subsection{Interacting particles in harmonic potentials: the quantum case}

In the classical model we have studied a back bending in the caloric curve appears evident. However, one may worry about the generality of such a statement. Do such anomalies also exist in quantum systems or is this definition of phase transition restricted to classical systems? In order to address this question we can solve the quantum analog of the model of section 4.3. Let us consider again A particles which can jump from one harmonic oscillator to another. In the first one all particles strongly interact while in the second one they are free. The curvature of the second well plays the role of a confining potential i. e. of a pressure. The corresponding Hamiltonian reads

$$
\hat{\mathbf{H}}=\omega_{1}\left(\hat{\mathbf{N}}_{1}-\frac{A_{1}}{2}\right)-\varepsilon\left(\hat{\mathbf{A}}_{1}^{2}-A_{t o t}^{2}\right)+\omega_{2}\left(\hat{\mathbf{N}}_{2}-\frac{A_{2}}{2}\right)
$$

with the operators

$$
\begin{aligned}
& \hat{\mathbf{N}}_{i}=\sum_{n=1}^{A} \delta_{i_{n}}^{i}\left(a_{n} a_{n}^{+}\right) \\
& \hat{\mathbf{A}}_{i}=\sum_{n=1}^{A} \delta_{i_{n}}^{i}
\end{aligned}
$$

where $i_{n}$ is the harmonic well occupied by the particle $i$. Using this Hamiltonian we can compute the level density and its associated entropy. To simplify the calculation we have chosen $\omega_{1}, \omega_{2}$ and $\varepsilon$ to be commensurable, $\omega_{2}=\omega_{1} / \lambda$ and $\varepsilon=\omega_{1} / \mu$. Then, we get the energy

$$
E / \varepsilon_{1}=N_{1}-\frac{A_{1}}{2}+\left(A_{\text {tot }}^{2}-A_{1}^{2}\right) / \mu+N_{2} / \lambda-\frac{A_{\text {tot }}-A_{1}}{2} / \lambda
$$

In a harmonic oscillator the number of states associated with $N_{1}$ quanta carried by $A_{1}$ particles is $\left(\begin{array}{l}N_{1}-1 \\ A_{1}-1\end{array}\right)$ so that for the double oscillator system this corresponds to

$$
W^{A_{t o t}}(E)=\left(\begin{array}{l}
A_{t o t} \\
A_{1}
\end{array}\right)\left(\begin{array}{l}
N_{1}-1 \\
A_{1}-1
\end{array}\right)\left(\begin{array}{l}
N_{2}-1 \\
A_{t o t}-A_{1}-1
\end{array}\right)
$$

Then, we can compute the temperature and the associated heat capacity (see figure 16). We observe that the system indeed presents an anomaly in the curvature of the entropy. Back-bending and negative heat capacities automatically follow.

From this analytical example of the occurrence of a negative heat capacity, we can come to the following conclusions:

1. negative heat capacity is not an artifact of imperfect numerical simulations: a $C<0$ system can be thermodynamically stable. 


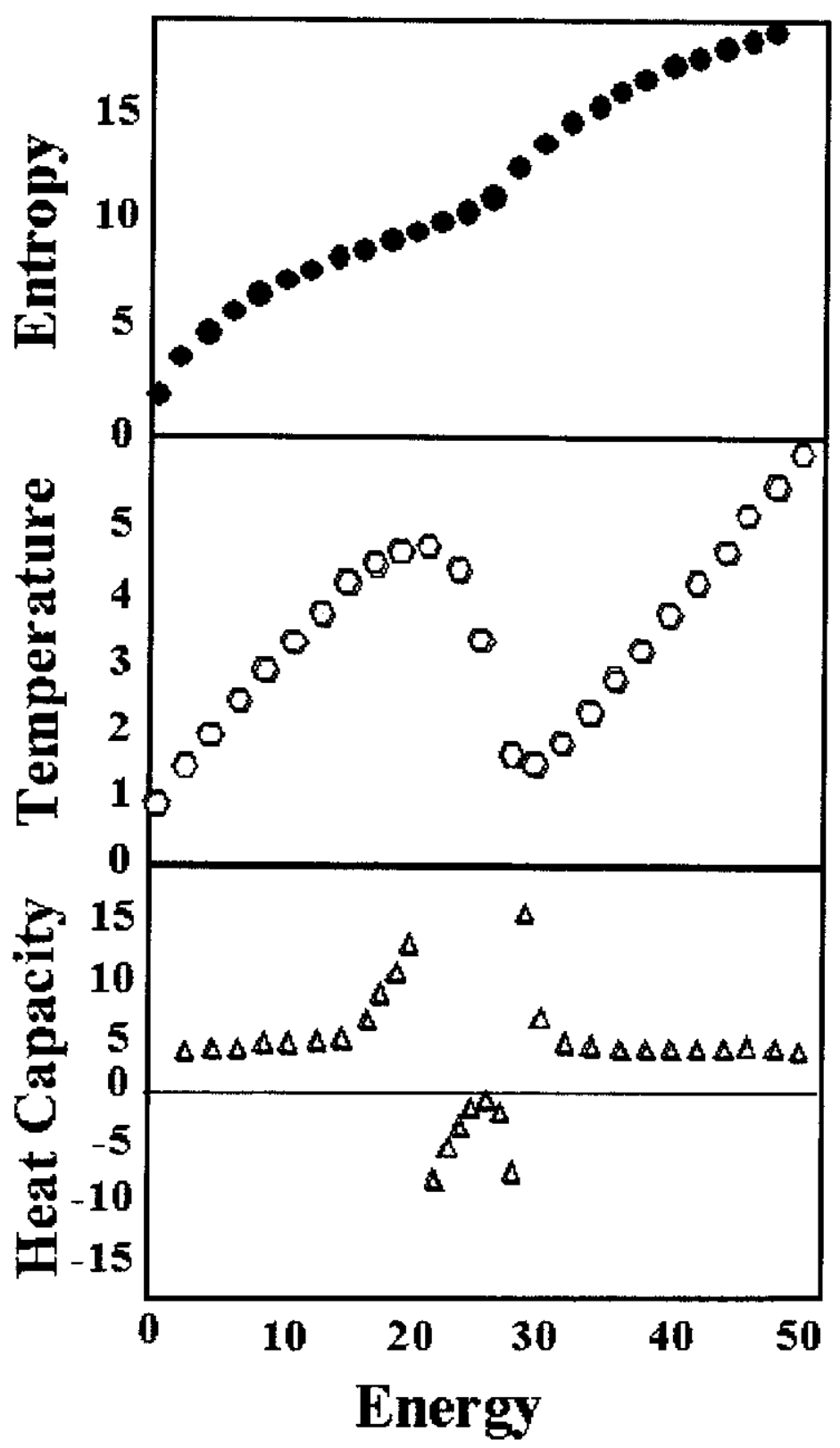

Fig. 16. Microcanonical entropy, temperature and heat capacity for the two quantum oscillators model in the subcritical regime. 
2. the existence of two different kinds of states for a system appears as a phase coexistence only if their respective level densities are sufficiently different (for instance particles interacting only in the low energy phase $(\varepsilon>0)$ and a much higher volume ( $\omega_{1} / \omega_{2}$ large) available for the high energy phase) so that their addition presents a concavity anomaly.

3 . if the average energy of the two phases is not the same (i.e. energy is an order parameter) a finite isolated system in equilibrium at phase coexistence presents a negative heat capacity.

\subsection{Surface tension and negative heat capacity}

We have seen that $C<0$ is a generic feature of finite systems at coexistence and is expected to disappear at the thermodynamical limit (at least if the interactions are short-ranged, see chapter 5). One can ask how much this behavior is confined to the microscopic world: how small a system has to be for the convex intruder to be sizeable? To answer to this question let us consider the macroscopic analytical example of a liquid drop in equilibrium with its vapor [19].

The bulk free energy of an incompressible liquid can be parametrized in the spirit of the Landau theory as $F=F_{0}+N k\left(v-v_{0}\right)^{2} / 2$ where $k$ is the compressibility, $v$ the reduced volume $v=V / N=\rho^{-1}$ and $v_{0}$ the saturation point. For a finite liquid drop one needs to introduce an additional term coming from the surface tension $\sigma$ leading to the free energy per particle of the drop $f_{L}=f_{0}+k\left(v-v_{0}\right)^{2} / 2+\sigma s N^{-1 / 3}$ where the surface coefficient $s=S N^{-2 / 3}$. The free energy of the vapor can be analytically calculated under the approximation that the vapor is an ideal classical monatomic gas. For $N$ indistinguishable non interacting particles in a volume $V$ we have $Z=z^{N} / N$ ! with the single particle partition sum

$$
z=V \frac{1}{h^{3}} \int d^{3} p \exp \left(-\beta \frac{p^{2}}{2 m}\right)=V\left(\frac{m \pi}{2 \beta \hbar^{2}}\right)^{3 / 2}
$$

which finally gives for the free energy per particle

$$
f_{G}=-T \ln v-\frac{3}{2} T \ln T+K
$$

The free energies of the two phases are schematically shown in figure 17. Phase coexistence implies the equality between the two partial pressures $\partial_{v} f_{L}=$ $\partial_{v} f_{G}$ which gives the usual tangent construction (dashed line). The finiteness of the system appears in the constraint of mass conservation. As we increase $v$ we dive inside coexistence with an increased proportion of the vapor fraction respect to the liquid fraction; this leads to an overall increase of the free energy of the drop and a consequent increase of the slope of the tangent construction as a function of $v$. The net result is a convexity anomaly of the free energy, i.e. a negative compressibility. The backbending in the $p(v)$ equation of state can be analytically calculated using the Clapeyron equation

$$
\frac{d p}{d T}=\frac{\Delta h}{\left(v_{G}-v_{L}\right) T}
$$




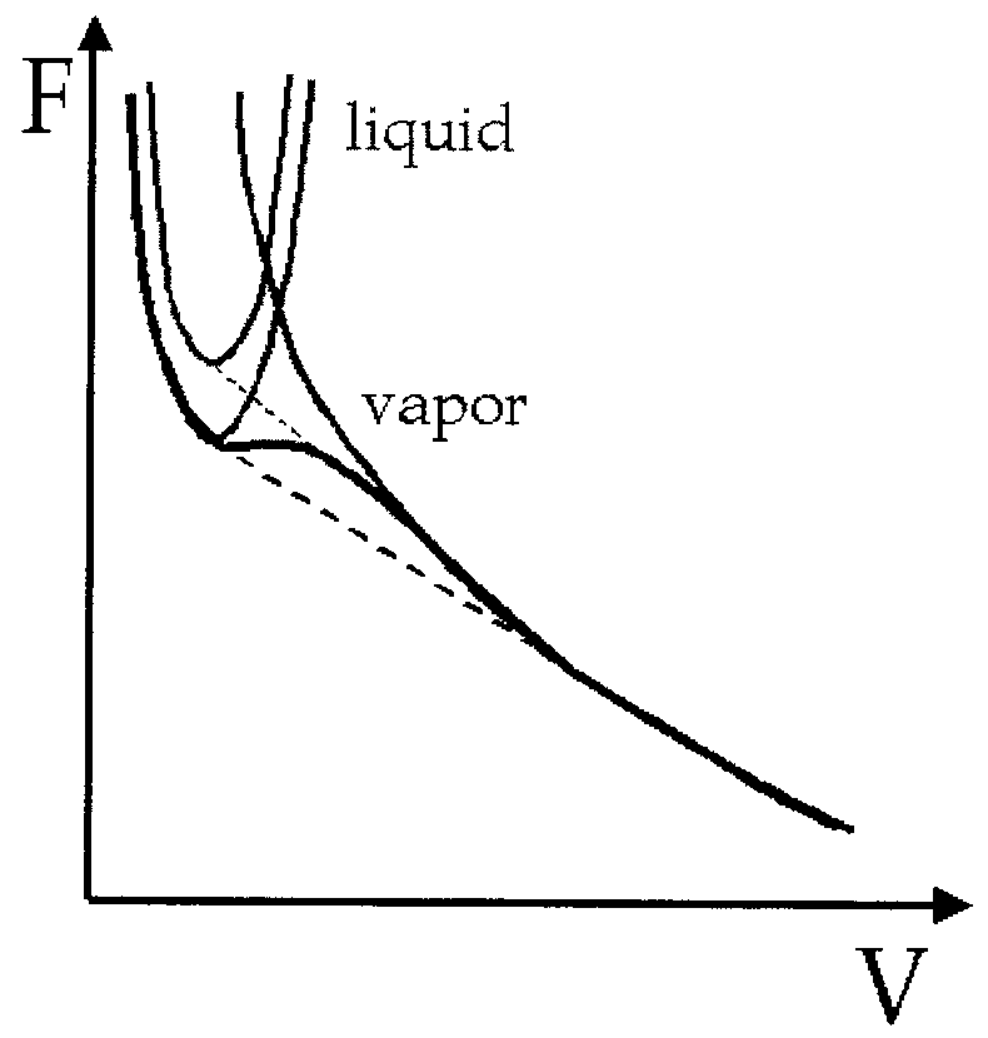

Fig. 17. Schematic representation of the free energy of a liquid drop of different sizes (parabolic curves) in equilibrium with its vapor.

where the enthalpy of vaporization per particle is given by $\Delta h=\Delta h_{0}-3 c_{s} v_{L} / r$, $c_{s}$ is the surface energy coefficient and $r$ is the drop radius. Assuming the bulk vaporization enthalpy $\Delta h_{0}$ as well as the specific volume of the drop $v_{L}$ as constant, the Clapeyron equation can be directly integrated giving

$$
p=p_{\text {bulk }} \exp \left(\frac{3 c_{s} v_{L}}{r T}\right)
$$

which grows as the drop radius decreases (and consequently the specific volume increases) showing that the compressibility is negative at coexistence even for macroscopic droplets, while the plateau is recovered in the bulk limit.

This schematic example shows that the convexity anomalies associated to first order phase transitions can be relevant even on a mesoscopic scale. An interesting consequence of that is that the value of physical observables can be drastically different in the different ensembles still at a mesoscopic scale. As 
an example, a caloric curve is always by definition monotonic in the canonical ensembles while we have seen that temperature can decrease for increasing excitation if the system is isolated. Of course the quantity called temperature is not defined in the same way in the two ensembles; as long as ensembles are not equivalent and $\beta \neq \partial_{E} \ln W$ we can wonder weather $\partial_{E} \ln W$ still represents the physical temperature of the system: does the anomaly that we have analytically recognized in the density of states really imply that pumping energy out of a system heats it up, or is it rather a mathematical curiosity? This question, addressed in the next section, can of course be generalized to any intensive variable conjugated to an order parameter in a generic first order phase transition.

\subsection{What is temperature?}

We all know that the second law of thermodynamics states that temperature measures the increase rate of entropy, $d S=d Q / T$ where $Q$ is the (disordered) thermal energy we have called $E$ in these lectures. Let us show that the rate of entropy is indeed the response of a thermometer loosely coupled to the system under study. In such a case loosely coupled means that the states of the total system are the independent tensorial products of the states of the thermometer times the one of the system. Moreover, the total energy is also simply the sum of the two partial energies.

$>$ From a macroscopic point of view, the equilibrium between the system and the thermometer requires the total entropy $S_{\text {tot }}=S_{s y s}+S_{\text {therm }}$ to be a maximum under the constraint of the total energy $E_{\text {tot }}=E_{\text {sys }}+E_{\text {therm }}$. The definition $T^{-1}=d S / d E$ leads then to the equality of temperatures for the system and the thermometer, $T_{\text {sys }}=T_{\text {therm }}$ in agreement with the zeroth's law of thermodynamics.

However we have also seen in the first chapter that starting from the same Shannon information kernel many different entropies exist according to the different constraints that define the equilibrium under study. The various entropies only converge toward a unique quantity at the thermodynamical limit if this latter exists. On the other side the quantity that backbends is only the microcanonical temperature $T^{-1}=d \ln W / d E$. We therefore ask the question weather a physical thermometer applied to an isolated system measures the microcanonical temperature, i.e. weather the negative heat capacity discussed above is a real measurable physical phenomenon. A thermometer by definition loosely interacts with the system. This means that if a thermometer (of energy $E_{\text {therm }}$ ) is put into a system (of energy $E_{\text {sys }}$ ), $E_{\text {therm }}$ and $E_{\text {sys }}$ can be considered as independent variables. The ensemble of system plus thermometer is isolated with a total energy $E_{\text {tot }}$, therefore the equiprobability of microstates and the factorization of the Hilbert space into the system and thermometer parts, leads to the equilibrium probability distribution for the thermometer energy

$$
p_{E_{t o t}}\left(E_{t h}\right)=\frac{W_{t h}\left(E_{t h}\right) W_{s y s}\left(E_{t o t}-E_{t h}\right)}{W_{t o t}\left(E_{t o t}\right)}
$$


where $W_{t h}\left(W_{\text {sys }}\right)$ is the number of states of the thermometer (system) and where $W_{\text {tot }}\left(E_{t o t}\right)=\int d E_{t h} W_{t h}\left(E_{t h}\right) W_{s y s}\left(E_{t o t}-E_{t h}\right)$ is the number of states of the total system (thermometer + system). Temperature is then defined as the response of the thermometer in the most probable state; if we maximize the probability (37) we get

$$
\frac{d \ln W_{t h}}{d E_{t h}}=\frac{d \ln W_{s y s}}{d E_{\text {sys }}}
$$

which shows that the quantity shared at the most probable energy partition is indeed the microcanonical temperature. This result is not in contradiction with the standard idea that for a thermostat, the physical temperature is the intensive variable conjugated to the energy, i.e. the (inverse of the) $\beta$ Lagrange multiplier. Indeed if we consider a thermometer inside a thermal bath its most probable energetic state will be given by the maximum of the canonical distribution of energies

$$
p\left(E_{t h}\right)=W_{t h}\left(E_{t h}\right) \exp \left(-\beta E_{t h}\right) / Z_{\beta}
$$

which gives an energy $\bar{E}_{t h}$ such that $\partial_{E} \ln W_{t h}\left(\bar{E}_{t h}\right)=\beta$

Equation (37) shows that from a microscopic point of view the temperature is indeed an ensemble property: the properties of a thermometer fluctuate from event to event. Only at the thermodynamical limit (for both the system and the thermometer) the fluctuations will be reduced to zero and the distribution of thermometer responses to a unique value.

An example of thermometer is given by the kinetic energy: indeed for a classical system eq.(37) holds with $E_{s y s}=E_{\text {pot }}$ and $E_{t h}=E_{k i n}$. Eq.(38) then implies that the most probable kinetic energy measures the microcanonical temperature.

\section{A typical finite system: the explosion of a supernova}

In the previous chapter we have shown that negative heat capacities systematically occur in first order phase transitions of finite systems when energy is an order parameter. From an historical point of view however, the problematics linked to negative heat capacity has started in the early seventies in a very different context, namely as a specificity of self-gravitating systems. Indeed a star that has exhausted its nuclear fuel radiates (i.e. loses energy) and heats up because of the gravitational contraction, i.e. behaves as a negative heat capacity system. Such a thermodynamical interpretation of the last steps of stellar evolution was considered as a triviality by the astronomers and as a absurdity by thermodynamicists. Let us summarize the two opposite arguments here.

- The astronomers' point of view. If we consider an isolated system with a potential energy $\propto r^{-n}$, the virial theorem states

$$
2 E_{k i n}+n E_{\text {pot }}=0
$$

In the case of the gravitational potential $n=1$ and we get for $N$ particles $E_{\text {tot }}=-E_{k i n}=-3 / 2 N T$ which implies $C=-3 / 2 N<0$ showing that a self-gravitating object has always $C<0$. 
- The thermodynamicists' point of view. For a generic equilibrated system in the canonical ensemble

$$
C=-\beta^{2} \frac{d<E>}{d \beta}=\beta^{2}\left\langle(E-<E>)^{2}\right\rangle>0
$$

1. showing that the heat capacity corresponds to the energy fluctuation which cannot be negative.

In the following sections we will show that both statements are incorrect, and that negative heat capacity in macroscopic self-gravitating systems is a physical equilibrium phenomenon exactly equivalent to the first order phase transitions in finite systems studied in the last chapter. Indeed a mesoscopic boiling droplet and an collapsing (or exploding) star are very similar in the sense that in both cases the size of the system is comparable to the range of the force.

\section{1 $C<0$ in self-gravitating systems and the gravothermal catastrophe}

The confusion arising from the two contradictory arguments about the sign of the heat capacity was increased by the famous gravothermal catastrophe predicted first by Antonov[20] from the study of the extrema of the microcanonical entropy of a self-gravitating object in the mean field approximation as a function of the one body matter density. The one body Shannon entropy reads

$$
S=-\int d^{3} r d^{3} p f(\vec{r}, \vec{p}) \ln f(\vec{r}, \vec{p})
$$

The microcanonical equilibrium can be found as usual by maximizing the entropy in a fixed volume $V$ under the particle number and energy constraint (see chapter 1)

$$
\begin{aligned}
& 0=d S-\alpha d N-\beta d E \\
& N=\int d^{3} r d^{3} p f(\vec{r}, \vec{p}) \\
& E=\int d^{3} r d^{3} p \frac{p^{2}}{2 m} f(\vec{r}, \vec{p})-\frac{G m^{2}}{2} \int d^{3} r d^{3} p d^{3} r^{\prime} d^{3} p^{\prime} \frac{f f^{\prime}}{\left|\vec{r}-\vec{r}^{\prime}\right|}
\end{aligned}
$$

The result is a self-consistent equation for the equilibrium one body matter density $\rho(\vec{r})=\int d^{3} p f(\vec{r}, \vec{p})$ that can be solved to give the density contrast $\rho_{c} / \rho_{e}$ between the center and the surface of the box. The result is that the density contrast is an increasing function of the volume. Figure 18 shows an exact calculation of this problem for the case of identical hard spheres[21]. For extremely large volumes $\rho_{c} / \rho_{e}>709$ the extremum of the entropy is a minimum meaning that no equilibrium solution exists in this case (Antonov gravothermal catastrophe). This result looks particularly strange since the instability provoked by the gravitational potential does not appear when the box is too small (and 


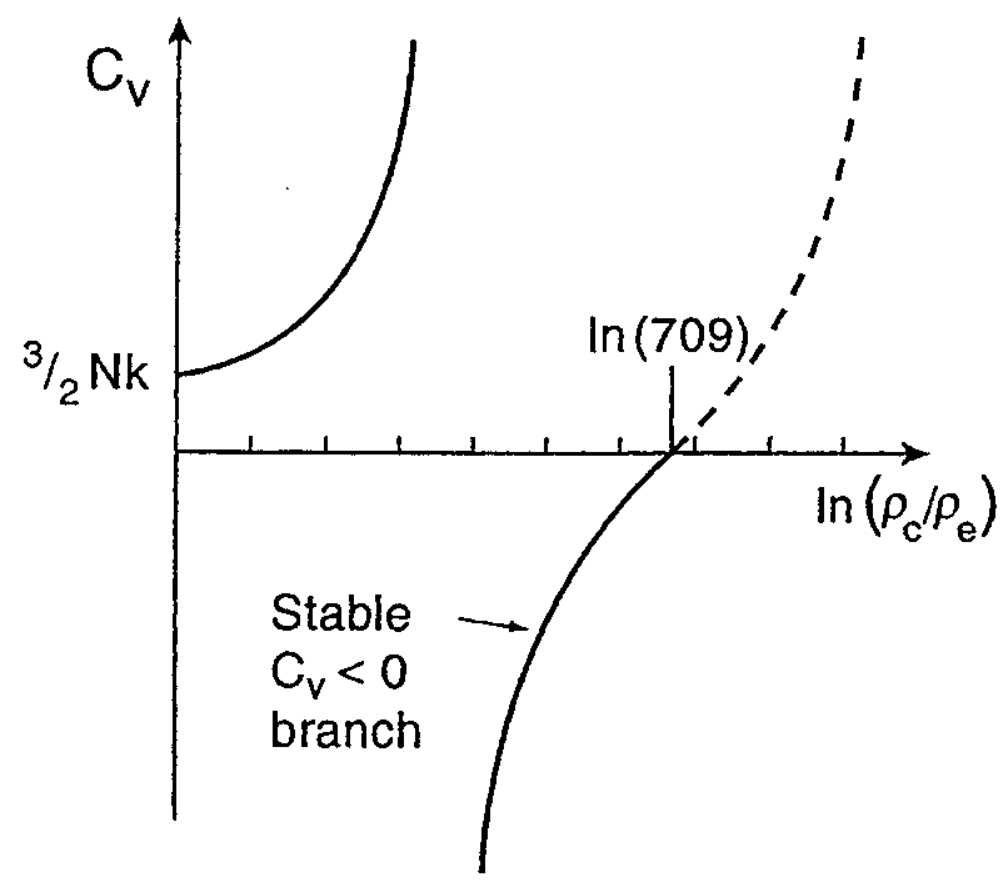

Fig. 18. Microcanonical heat capacity as a function of the density contrast for hard spheres with gravitational interactions from ref.[21].

the interaction energy is strong) but when the box is too large (and the interaction energy is loose). Moreover for intermediate boxes $32.2<\rho_{c} / \rho_{e}<709$ the extremum is a maximum, i.e. the solution is stable, but the heat capacity contains a pole and a negative branch, which traditionally was associated to an instability (see chapter 3 ).

\subsection{Solution of the Antonov paradox}

The disagreement between the gravothermal catastrophe and eq.(40) clearly implies that the Van Hove theorem (see section 4.1) is violated. This can only be due to the long range of the force. The incoherence with the virial theorem eq.(39) can be explained by considering that if we are dealing with $N$ particles the density of states (see section 4.3 )

$$
W \propto \int d^{3 N} r\left(E-\sum_{i j} \frac{m_{i} m_{j}}{r_{i j}}\right)^{3 N / 2}
$$

diverges unless a short range cut-off and a constraining potential (or boundary condition) are introduced. This means that the virial theorem has to be corrected for the short range repulsion and for the boundary condition. A schematic 
modelization of this problem has been proposed by $\mathrm{W}$. Thirring as a constant interaction among all particles inside a volume $V_{0}<V$ and no interaction outside[22]. This model is equivalent to the two coupled harmonic oscillator problem proposed in section 4.3. The finite liquid drop of section 4.3 is now an infinite star, while the saturated vapor corresponds to the star atmosphere. As for the model 4.3 , for the two boxes model a $C<0$ is found [22] whenever the difference between the two volumes is important, i.e. for big total volumes in agreement with the Lynden-Bell result[21]. Following the reasoning of chapter 4 we can then associate the $C<0$ regime to a phase coexistence between the star and its atmosphere that stabilizes the stellar nuclear plant, and the gravothermal catastrophe to a first order phase transition when the nuclear fuel is exhausted.

If we consider the generic case for the interaction in the dense phase $E=$ $\varepsilon N^{\gamma}$ we can study the effect of the range of the force on the heat capacity in the thermodynamical limit. The resulting caloric curves are given in figure $19[22]$. In agreement with chapter 4 , we can see that the negative heat capacity disappears at the thermodynamical limit for short range interactions $(\gamma=1)$ but all ranges $\gamma>1$ lead to a backbending that is preserved when $N \rightarrow \infty$, i.e. this phenomenon is not specific of the gravitational interaction. We can also understand why in the short range case the same phenomenology appears in selfgravitating macroscopic system, as long as the range of the force is comparable with the linear dimension of the system.

\subsection{Thermal contact between $C<0$ systems}

Phase coexistence in standard macroscopic thermodynamics is a trivial phenomenon: the tangent construction implies that the thermodynamical properties of phase coexistence are completely determined by the properties of the corresponding pure phases. On the other hand if surfaces cannot be neglected respect to bulk properties (i.e. if the system is finite or the interaction is long range) new unexpected phenomena peculiar to the coexistence phase appear, as negative compressibility or negative heat capacity. The peculiar thermodynamical properties of the coexistence phase can be better understood if we consider in some detail the problem of thermal contact between $C<0$ systems.

Let us consider a system with $C_{1}<0$ in thermal contact with a second system with a heat capacity $C_{2}$. Let us distinguish some different cases.

1. If $C_{2}<0$ no equilibrium is possible between the two systems. Indeed if $T_{2}>T_{1}$ energy will be transferred from system (2) to system (1) and system (2) will get even hotter while (1) becomes even cooler. This implies that it is not possible to divide a $C<0$ system into two parts each with $C<0$; in other words $C<0$ systems are not extensive.

2. A thermal equilibrium is possible if the $C_{1}<0$ system is in contact with a $C_{2}>0$ system small enough such that $C_{2}<\left|C_{1}\right|$. In this case the temperature increase of the initially slightly hotter system (1) is compensated by system (2) which has positive heat capacity and receives energy, and therefore increases its temperature more rapidly; a final equilibrium state will 


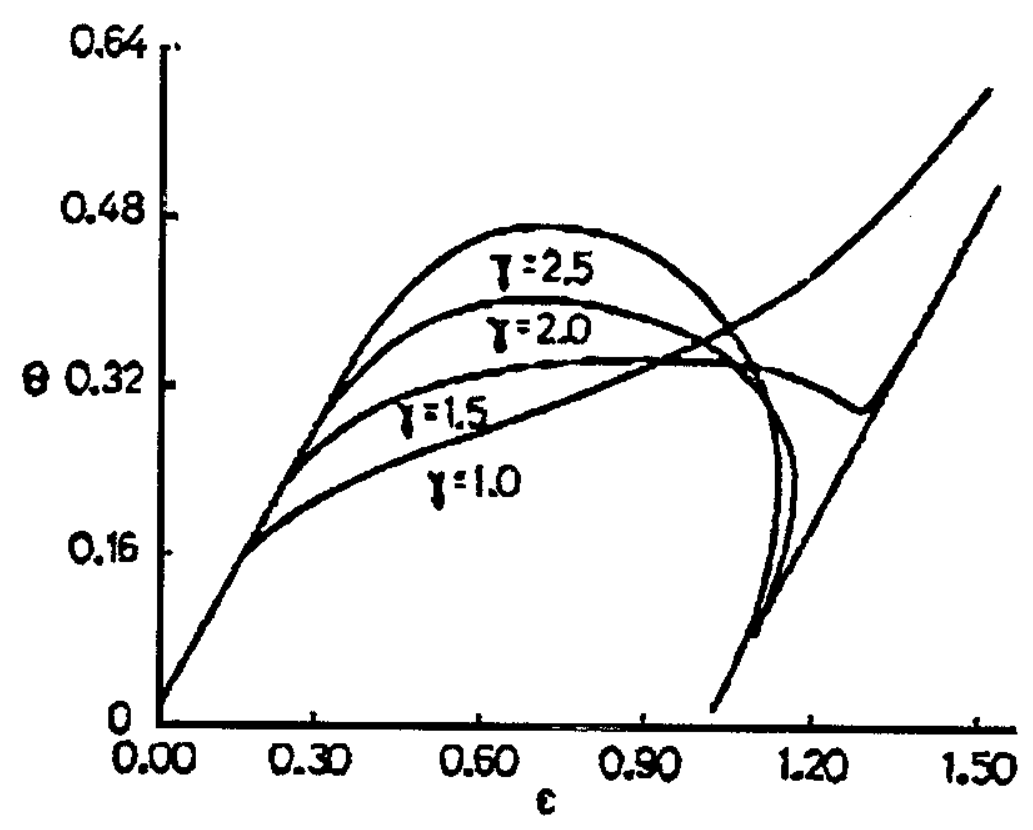

Fig. 19. Caloric curves in the subcritical regime for different ranges of the interaction from ref.[22].

then be achieved with $T_{e q}>\max \left(T_{1}, T_{2}\right)$.In particular if the two systems are independent we can write for the microcanonical distribution of energy $E_{1}: \ln P_{E}\left(E_{1}\right)=S_{1}\left(E_{1}\right)+S_{2}\left(E_{2}\right)-S_{\text {tot }}(E)$ where $E=E_{1}+E_{2}$. The stability condition (i.e. the curvature of $\ln P$ around the extrema) can then be written as

$$
\frac{d^{2} S_{t o t}}{d E_{1}^{2}}=-\frac{1}{2 T^{2}}\left(\frac{1}{C_{1}}+\frac{1}{C_{2}}\right)<0
$$

where $T_{1}=T_{2}=T$ is the equilibrium temperature (the microcanonical temperature associated with the most probable energy partition). This is the case of a living star: the central part has $C_{1}<0$, the surface has $C_{2}>0$, the global system (or coexistence phase) has $C=C_{1}+C_{2}<0$ and is stable since the core transfers energy to the atmosphere in the form of radiation. The gravothermal catastrophe occurs when $C=0$ (see figure 18) when the gravitational contraction cannot be stopped by nuclear reactions leading to the whole well known phenomenology of stellar collapse[23].

3. A $C<0$ system cannot reach thermal equilibrium with a thermostat. Indeed if (1) is much smaller than (2) eq.(41) is violated and the total entropy is a minimum. This implies that a negative heat capacity is impossible in the canonical ensemble coherently with eq. $(40)$ : the $C<0$ system in contact with a thermal bath stops to hesitate between the two coexisting phases and 
jumps to its stable equilibrium state, i.e. makes a phase transition. These considerations allow to gain an intuitive understanding of the fact that the $C<0$ of a boiling liquid drop (see chapter 4) transforms into a plateau when a water kettle is put on a gas cooker in the macroscopic world. Indeed any microscopic portion of the boiling portion once isolated would appear as a microcanonical $C<0$ system; the thermal contact with the rest of the system (which can be considered a thermostat because of the short range of the force) forces the stable $C<0$ system to choose between the liquid and the gas solution giving rise to the Maxwell construction.

All we have discussed in this chapter has been presented in the early seventies; it may be surprising then that negative heat capacity has not been recognized at this time as a general paradigm of phase coexistence in non-extensive (finite or infinite) systems. Indeed it is only in the recent years, following the success of the experimental application of these ideas to mesoscopic systems (see chapters 7 and 8) that these concepts have been developed further. The conceptual difficulty of accepting a stable equilibrium with negative heat capacity is due to different reasons.

First, the inhomogeneity of the star makes it difficult to consider it as a single thermodynamical object; following the reasoning of chapter 4 we however understand that on the contrary this inhomogeneity is an essential feature of the thermodynamics of coexisting phases.

Moreover the fact that the energy exchange between the star and the atmosphere consists in radiation (taking their energy from nuclear reactions) makes the problem an out of equilibrium transport problem.

Most important, the fact that it is not possible to define a canonical equilibrium for a $C<0$ system means that the validity itself of such an equilibrium is not easy to understand: how can one speak of temperature in the absence of thermal contact, and what is in this case the meaning of a temperature decrease with increasing energy? We have already discussed (and solved!) the problem of the physical meaning of the microcanonical temperature in section 4.5 ; let us just recall here that if a $C<0$ cannot be put in contact with a thermostat, on the other hand a thermometer put in a $C<0$ system is perfectly well defined, and measures the microcanonical temperature. This is the case 2 of the discussion above (with $C_{1}<0$ and $C_{2}<\left|C_{1}\right|$ )

\section{Abnormal topology of event distributions}

In most textbooks the equivalence between the different statistical ensembles is either postulated or demonstrated at the thermodynamical limit through the Van Hove theorem (see section 4.1).

In the previous chapters we have shown that ensembles may not be equivalent. For finite systems, two ensembles which put different constraints on the fluctuations of the order parameter lead to very different equations of states close to a first order phase transition. As an example the microcanonical heat capacity 
may diverge to become negative while the canonical one remains always positive and finite (see sections $4.2,4.3$ ). In chapter 5 we have moreover seen that such inequivalences may survive at the thermodynamical limit for systems involving long range forces.

In this chapter we will try to formalize all these findings looking at the general properties of the order parameter distribution. This will allow us to propose a definition of phase transitions in finite systems also for intensive ensembles (i.e. ensembles where the order parameter is not controlled on an event by event basis ) based on topology anomalies of the event distribution in the space of observations. We will show in the next sections that this generalizes the definitions based on the curvature anomalies of thermostatistical potentials presented in chapter 4 . Such a definition gives a clear understanding of the physical meaning of an order parameter as the best variable to separate the two maxima of the distribution, and can be directly used experimentally (see chapters 7,8 ).

In section 6.6 we will come back to the problem of the thermodynamical limit. If the order parameter is sufficiently collective the anomaly represented by the phase transition may survive until the infinite volume and infinite number limit. According to the specific properties of the Hamiltonian we will then work out a sufficient condition for the finite size phase transition to become the one known in the bulk.

\subsection{Negative heat capacity and bimodal energy distributions}

Let us first concentrate on finite systems. To begin we will consider the specific example of the microcanonical and the canonical ensemble characterized by the energy $E$ and the temperature $\beta^{-1}$ respectively.

In section 4.5 we have shown that the extrema of the probability distribution in the canonical ensemble

$$
P_{\beta}(E)=\exp \left(S(E)-\beta E-\log Z_{\beta}\right)
$$

are given by the equation

$$
T^{-1} \equiv \partial_{E} S\left(\bar{E}_{\beta}\right)=\beta
$$

If this extremum is unique, we can perform a saddle point approximation around the most probable energy $\bar{E}_{\beta}$ leading to the average energy

$$
\langle E\rangle_{\beta}=\int d E E e^{-\frac{\left(E-\bar{E}_{\beta}\right)^{2}}{2 C}} g_{\beta}(E-\bar{E})
$$

with $g_{\beta}(x)=c_{0}+c_{3} x^{3}+c_{4} x^{4}+\ldots$ If $P_{\beta}$ is symmetric, $\langle E\rangle_{\beta}=\bar{E}_{\beta}$ and $T^{-1}(<$ $E>)=. \beta$ meaning that the microcanonical caloric curve $T(\bar{E})$ exactly coincides with the canonical one $\beta^{-1}(\langle E\rangle)$. If the distribution is not symmetric some corrective terms arise : $\langle E\rangle_{\beta}=\bar{E}_{\beta}+\delta_{\beta}$, where $\delta_{\beta}=\int d x x \exp \left(-x^{2} / 2 C\right) \tilde{g}_{\beta}(x)=$ $3 c_{3} \sqrt{2 \pi C^{5}}+\ldots$ with $\bar{g}_{\beta}$ the series of the odd terms of $g_{\beta}$. However, the shift $\delta$ is in most cases small so that when $P_{\beta}$ has a unique maximum the ensembles 
are almost equivalent even for a finite system. The same approximation can be applied to the partition sum, which is linked to the entropy by an exact Laplace transform

$$
Z_{\beta}=\int d E W(E) e^{-\beta E}
$$

leading to

$$
Z_{\beta} \approx W(\bar{E}) e^{-\beta \bar{E}} \approx W(<E>) e^{-\beta<E>}
$$

which corresponds to a simple linear transformation between the thermodynamical potentials, i.e. an approximate Legendre transform

$$
\ln Z_{\beta} \approx \ln W(<E>)-\beta<E>
$$

As we have already mentioned in section 2.1 , this expression has not to be mixed up with the true (and exact) Legendre transform $\ln Z_{\beta}=S_{\beta}(\langle E\rangle$ ) $-\beta<E>$ which gives the relation between the partition sum and the Shannon entropy within the canonical ensemble.

However in first order phase transitions $P_{\beta}$ has a characteristic bimodal shape (see section 2.2) [24-26] with two maxima $\bar{E}_{\beta}^{(1)}, \bar{E}_{\beta}^{(2)}$ that can be associated with the two phases and a minimum $\bar{E}^{(0)}$. These three solutions of Eq.(42) imply a backbending for the microcanonical caloric curve. Indeed a minimum of $P_{\beta}$ corresponds to a convexity of the entropy according to $d_{E}^{2} \ln P_{\beta}=d_{E}^{2} \ln W$. A single saddle point approximation is not valid in this case; however it is always possible to write $P_{\beta}=m_{\beta}^{(1)} P_{\beta}^{(1)}+m_{\beta}^{(2)} P_{\beta}^{(2)}$ with $P_{\beta}^{(i)}$ mono-modal normalized probability distribution peaked at $\bar{E}_{\beta}^{(i)}$. The canonical mean energy is then the weighted average of the two energies

$$
\langle E\rangle_{\beta}=\tilde{m}_{\beta}^{(1)} \bar{E}_{\beta}^{(1)}+\tilde{m}_{\beta}^{(2)} \bar{E}_{\beta}^{(2)}
$$

with $\tilde{m}_{\beta}^{(i)}=m_{\beta}^{(i)} \int d E P_{\beta}^{(i)}(E) E / \vec{E}_{\beta}^{(i)} \simeq m_{\beta}^{(i)}$, the last equality holding for symmetric distributions $P_{\beta}^{(i)}$. Since only one mean energy is associated with a given temperature $\beta^{-1}$, the canonical caloric curve is monotonous. In particular in the backbending region the mean energy is an interpolation between the two energies $\bar{E}_{\beta}^{(1)}, \bar{E}_{\beta}^{(2)}$ associated with the considered $\beta$, the weighting factor being the probability of each phase $\tilde{m}_{\beta}^{(i)}$.

If instead of looking at the average $\langle E\rangle_{\beta}$ we look at the most probable energy $\bar{E}_{\beta}$, this (unusual) canonical caloric curve is almost identical to the microcanonical one (see eq. (42)) up to the transition temperature $\beta_{t}^{-1}$ for which the two components of $P_{\beta}(E)$ have the same height. At this point the most probable energy jumps from the low to the high energy branch of the microcanonical caloric curve. The most probable canonical energy is still a monotonic curve but it presents a plateau at $\beta_{t}^{-1}$ which is equivalent to the Maxwell construction since 


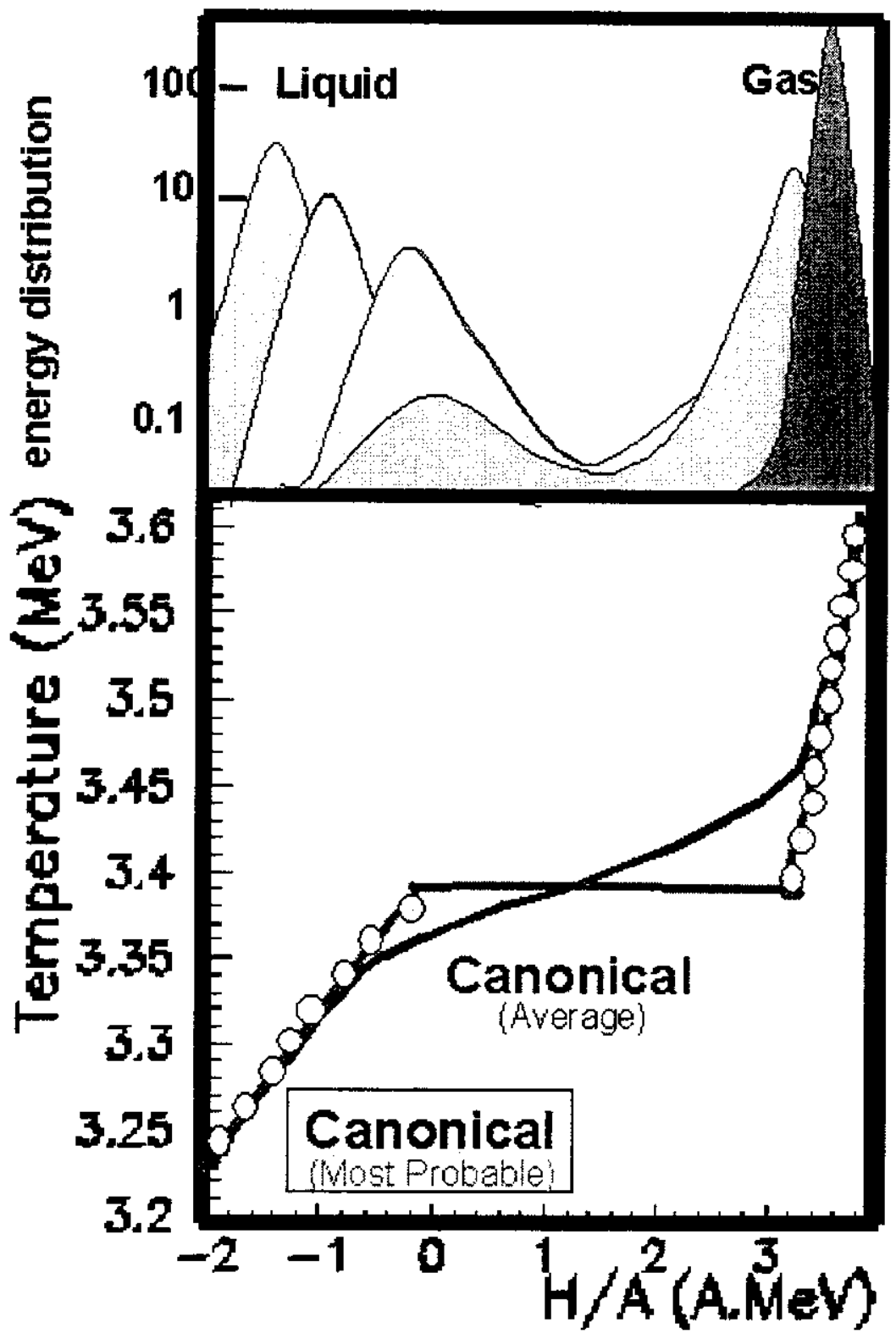

Fig. 20. Canonic thermodynamics of 216 particles in the isobar Lattice Gas model. Upper part: energy distributions for different temperatures. Lower part: caloric curve from the average and the most probable energy. 


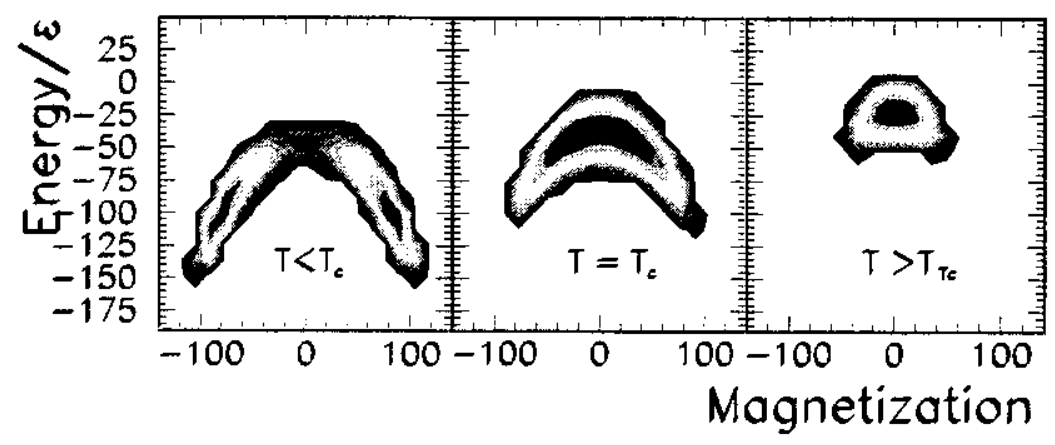

Fig. 21. Event distribution in the energy versus magnetization plane for a $6 \times 6 \times 6$ Ising model with zero field at a subcritical, critical and supercritical temperature.

$$
S\left(\bar{E}_{\beta}^{(2)}\right)-S\left(\bar{E}_{\beta}^{(1)}\right)=\int_{\bar{E}_{\beta}^{(1)}}^{\bar{E}_{\beta}^{(2)}} \frac{d E}{T}=\beta\left(\bar{E}_{\beta}^{(2)}-\bar{E}_{\beta}^{(1)}\right)
$$

Therefore, the difference between the canonical and microcanonical caloric curves remains when one is looking at the most probable energy instead of the average. The connection between the canonical energy distribution and the microcanonical equation of state is presented for the three dimensional lattice gas model in figure 20. The bimodality of the canonical energy distribution as well as the discontinuity in the most probable energy value are definitions of the phase transition exactly equivalent to the convexity anomaly of the entropy discussed in chapter 4 and clearly defined even for very small system (216 particles are considered for the calculation of figure 20 ).

\subsection{Convexity anomalies and bimodal probability distributions}

This discussion can be generalized to any couple of extensive/intensive ensemble. Figure 21 shows the example of the Ising model at zero field. The bimodal structure in the $m$ direction corresponds to a negative susceptibility in a constant magnetization ensemble. In this case the projection on the energy axis does not show anomalies : the microcanonical heat capacity remains positive and the energy is not an order parameter. At $T_{c}$ the distribution presents a curvature anomaly only on the low energy side respect to the maximum. Therefore at this point the curvature passes through zero signalling a second order phase transition. Since in finite systems the canonical distribution for any $\beta, h(\mu)$ allows a complete exploration of the microcanonical entropy surface (in the limit of the total number of events analyzed), the whole microcanonical phase diagram can in principle be drawn from any single canonical temperature using the fact that the distribution is in fact the entropy

$$
S(E)=\log P_{\beta, h=0}(E, M)+\ln Z_{\beta, h=0}+\beta E .
$$


As an example the croissant shape of the distribution at $T_{c}$ not only defines the critical energy $e_{c}$ and magnetization $m_{c}$ of the second order phase transition but also allows to infer the coexistence line where the first order phase transition takes place. Indeed a constant energy cut of the distribution below $e_{c}$ directly represents the entropy as a function of magnetization and has a bimodal shape.

\subsection{Liquid gas phase transition and volume fluctuations}

Let us now take the example of the liquid-gas phase transition in a system of $n$ particles for which the volume is not controlled on an event by event basis but is at best known in average. In such a case, we can define an observable $\hat{B}_{1}$ as a measure of the size of the system; for example the cubic radius $\hat{B}_{1}=\frac{4 \pi}{3 n} \sum_{i} \hat{r}_{i}^{3} \equiv$ $\hat{V}$ where the sum runs over all the particles. Then a Lagrange multiplier $\lambda_{V}$ has to be introduced which has the dimension of a pressure divided by a temperature. In a canonical ensemble with an inverse temperature $\beta$ we can define different distributions which are illustrated in Fig. 22. A complete information is contained in the distribution $P_{\beta \lambda_{v}}(e, v)=\bar{W}(e, v) Z_{\beta \lambda_{v}}^{-1} \exp -\left(\beta e+\lambda_{v} v\right)$ since events are sorted according to the two thermodynamical variables, $e$ and $v$. This leads to the density of states $\bar{W}(e, v)$ with a volume $v$ and an energy $e$. One can see that in the first order phase transition region the probability distribution is bimodal. In the spirit of the principal component analysis we can look for an order parameter $\hat{Q}=x \hat{H}+y \hat{V}$ which provides the best separation of the two phases. A projection of the event on this order parameter axis is also shown in Fig.22. One can see a clear separation of the two phases. On the other hand if we cannot measure both the volume $v$ and the energy $e$ we are left either with

$$
P_{\beta \lambda_{v}}(e)=\bar{W}_{\lambda_{v}}(e) Z_{\beta \lambda_{v}}^{-1} \exp (-\beta e)
$$

giving access to the microcanonical partition sum $\tilde{W}_{\lambda_{v}}(e)$ at constant $\lambda_{v}$ or with the probability

$$
P_{\beta \lambda_{v}}(v)=\bar{Z}_{\beta}(v) Z_{\beta \lambda_{v}}^{-1} \exp \left(-\lambda_{v} v\right)
$$

leading to the isochore canonical partition sum $\vec{Z}_{\beta}(v)$. Since both probability distribution $P_{\beta \lambda_{v}}(e)$ and $P_{\beta \lambda_{v}}(v)$ are bimodal the associated partition sum do have anomalous concavity intruders, i.e. negative heat capacity as well as negative compressibility.

As a general statement, we can define a first order phase transition for any number of particles as a bimodality in the probability distribution of an arbitrary observable; any observable that allows to separate the two maxima of the event distribution can then be considered as an order parameter.

\subsection{A mesoscopic example: negative magnetic susceptibility}

To better explore the connection between the distribution of the order parameter and the equation of state, let us consider the Ising model (see section 2.3) at 


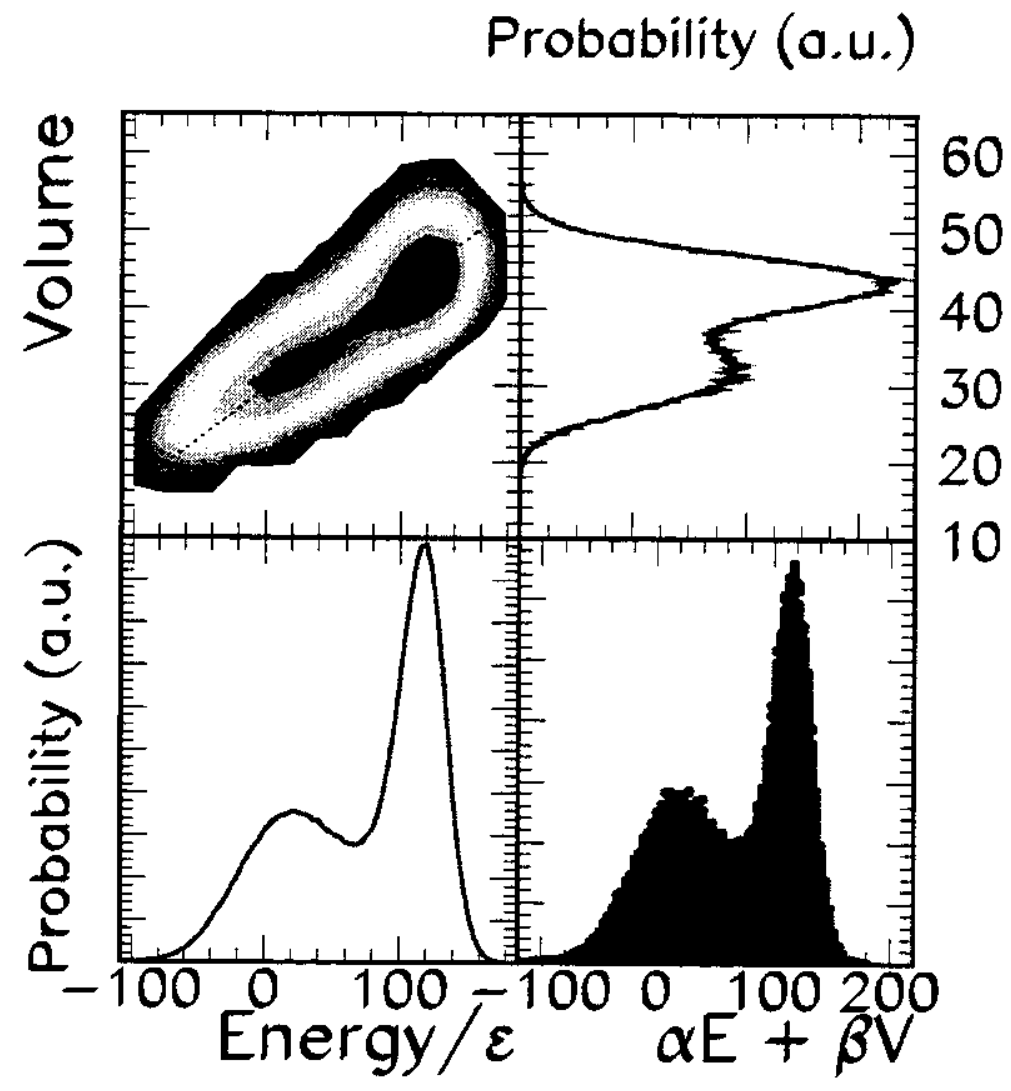

Fig. 22. Event distribution in the volume versus energy plane for 216 particles in the isobar canonical Lattice Gas model and projection over the two axes. Bottom right: projection of the distribution over the direction separating at best the two distributions.

fixed magnetization. The partition sum can be written as

$$
Z_{\beta}(m)=\int d E W(E, m) \exp (-\beta E)
$$

where $m=\sum_{i}^{N} s_{i} / N$ is the magnetization. As shown in figure 23 the phase transition is signalled by a backbending of the equation of state $\tilde{h}(m)=\beta^{-1} \partial_{m} \ln Z_{\beta}$ $(m)$ that replaces the discontinuity in the equation of state $\langle m\rangle(h)$ of the standard Ising model with partition sum

$$
Z_{\beta h}=\int d m d E W(E, m) \exp (-\beta(E+m N h))
$$




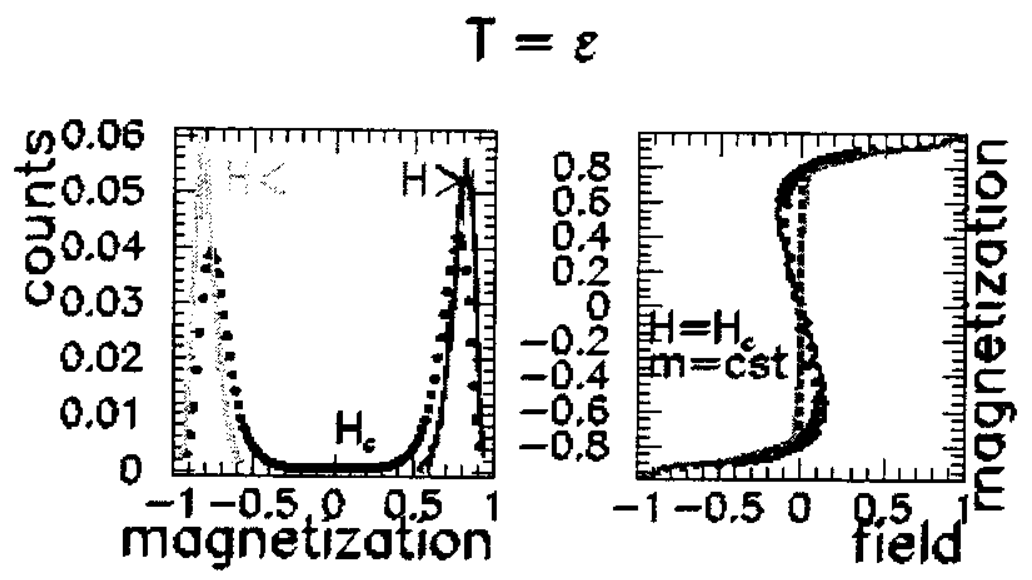

Fig. 23. Left side: magnetization distribution in a $6 \times 6 \times 6$ Ising model at a subcritical temperature for three different values of the external field. Right side: equation of state for Ising and for the constant magnetization ensemble (backbending curve).

Note that in the case of this specific model finite size correction are particularly small and an almost perfect jump is observed already for a $3 D$ lattice size of linear dimension $L=6$.

This same information concerning a negative magnetic susceptibility can be obtained by looking at the magnetization distribution of the standard Ising model with zero field

$$
P_{\beta h}(m)=\frac{1}{Z_{\beta h}} \int d E W(E, m) \exp (-\beta(E+m N h))=\frac{Z_{\beta}}{Z_{\beta h}} \exp (-\beta m N h)
$$

$>$ From equation (46) we can see that $d_{m}^{2} \ln N_{\beta h}=d_{m}^{2} \ln Z_{\beta}$. This shows that a minimum in the magnetization distribution (left part of fig.23) implies a convexity anomaly in the constant magnetization partition sum (right part of fig.23) i.e. a negative magnetic susceptibility for mesoscopic ferromagnets sorted in constant magnetization bins.

\subsection{First order phase transitions and bifurcations}

Another way to understand a first order phase transition is to relate it with a bifurcation. Let us for example look at the grancanonical lattice gas model at the critical chemical potential and let us look at the mass distribution as a function of the temperature (see figure 24). Above the critical temperature the mass distribution is normal but at the critical temperature it bifurcates into a bimodal distribution with two peaks:

- the gas one at low mass, i.e. low density,

- and the liquid phase at high mass.

This sudden bifurcation is typical of a phase transition. 


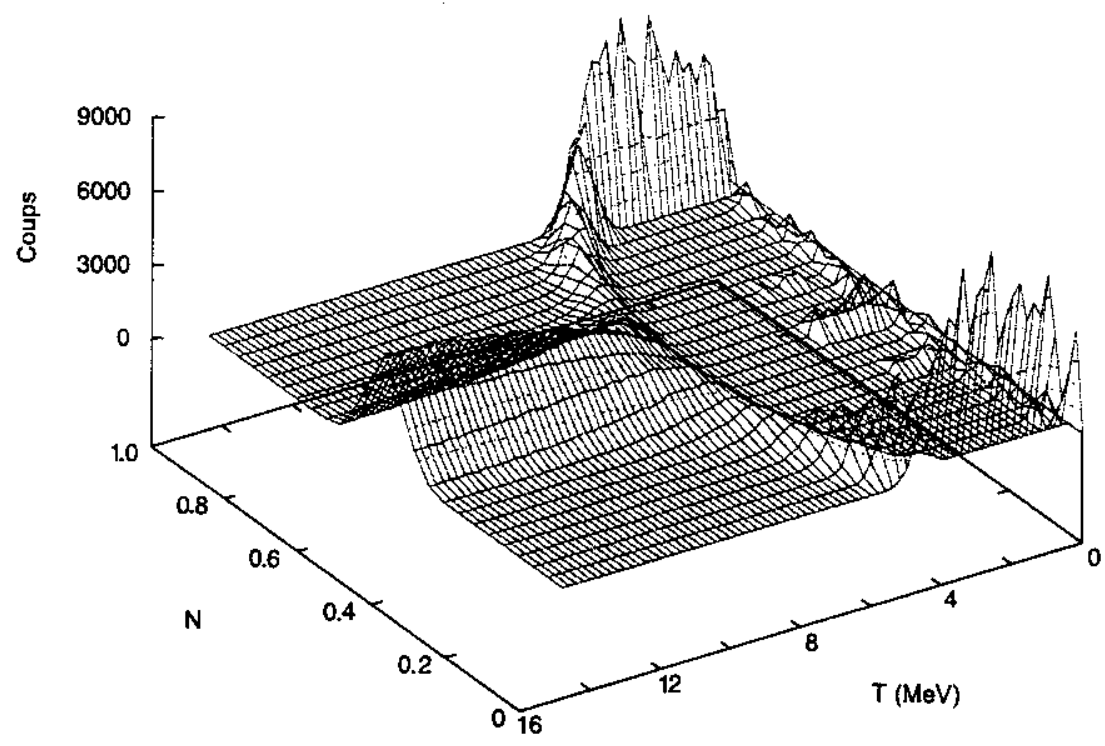

Fig. 24. Mass distribution as a function of the system temperature for the grancanonical lattice gas model.

\subsection{The thermodynamical limit and the Yang Lee theorem}

The definition of phase transition proposed in section 6.1 can be applied to a wide range of situations even out of equilibrium[27] and can be directly implemented on experimental data (see chapter 7,8 ). However it is clear that not all topological anomalies will survive up to the bulk limit and give rise to a conventional thermodynamical phase transition. The transition between two isomeric states, the breaking of a Cooper pair, ionization are all examples of state changes that do not lead to discontinuities in the bulk limit, i.e. do not converge to a thermodynamical first order phase transition. It is therefore of extreme interest to study the thermodynamical limit of the order parameter distributions. Since we have seen that the different statistical ensembles are in general not equivalent, the thermodynamical limit has to be considered separately for the intensive (say, canonical) and extensive (say, microcanonical) ensemble.

The thermodynamical limit can be expressed as the fact that the thermodynamical potentials per particle converge when the number of particles $N$ goes to infinity : $f_{N, \beta}=\beta^{-1} \log Z_{\beta} / N \rightarrow \bar{f}_{\beta}$ and $s_{N}(e)=S(E) / N \rightarrow \bar{s}(e)$ where $e=$ $E / N$. Let us also introduce the reduced probability $p_{N, \beta}(e)=\left(P_{\beta}(N, E)\right)^{1 / N}$ which then converges towards an asymptotic distribution $p_{N, \beta}(e) \rightarrow \bar{p}_{\beta}(e)$ where $\tilde{p}_{\beta}(e)=\exp \left(\bar{s}(e)-\beta e+\bar{f}_{\beta}\right)$. Since $P_{\beta}(N, E) \approx\left(\bar{p}_{\beta}(e)\right)^{N}$ one can see that when $\bar{p}_{\beta}(e)$ is normal the relative energy fluctuation in $P_{\beta}(N, E)$ is suppressed by a factor $1 / \sqrt{N}$. At the thermodynamical limit $P_{\beta}$ reduces to a $\delta$-function and the ensemble equivalence is recovered (see section 2.2). To analyze the thermodynamical limit of a first order phase transition (bimodal $p_{N, 3}(e)$ ), let us introduce 
as before $\beta_{N, t}^{-1}$, the temperature for which the two maxima of $p_{N, \beta}(e)$ have the same height. For a first order phase transition $\beta_{N, t}^{-1}$ converges to a fixed point $\bar{\beta}_{t}^{-1}$ as well as the two maximum energies $e_{N, \beta}^{(i)} \rightarrow \bar{e}_{\beta}^{(i)}$. For all temperature lower (higher) than $\bar{\beta}_{t}^{-1}$ only the low (high) energy peak will survive at the thermodynamical limit since the difference of the two maximum probabilities will be raised to the power $N$. Therefore, below $\bar{e}_{\beta}^{(1)}$ and above $\bar{e}_{\beta}^{(2)}$ the canonical caloric curve coincides with the microcanonical one in the thermodynamical limit. In the canonical ensemble the temperature $\bar{\beta}_{t}^{-1}$ corresponds to a discontinuity in the state energy irrespective of the behavior of the entropy between $\bar{e}_{\beta}^{(1)}$ and $\bar{e}_{\beta}^{(2)}$. A more rigorous demonstration can be done with the help of the Yang Lee unit circle theorem [26].

The Yang Lee theorem [28] considers the distribution of the zeros of the partition sum $Z_{\beta}$ in the complex $\beta$ plane. Under very general conditions it is possible to demonstrate [28] that the zeros form a line that cuts across the real axis with a density increasing with the number of particles of the system, leading to a vanishing imaginary part for $N \rightarrow \infty$, i.e. a first order phase transition at a definite (real) temperature. The partition sum for a complex parameter $\beta=\beta+i \eta$ is nothing but the Laplace transform of the probability distribution $P_{\beta_{0}}(e)$ for a temperature parameter $\beta_{0}[29,30]$

$$
Z_{\beta}=\int d e Z_{\beta_{0}} P_{\beta_{0}}(e) e^{-\left(\beta-\beta_{0}\right) e} . \equiv \int d e p_{\beta}(e) e^{-i \eta e}
$$

In order to study the thermodynamical limit (when it exists), if $p_{\beta}(e)$ is monomodal we can use a saddle point approximation around the maximum $\bar{e}_{\beta}$ giving $Z_{\beta}$. $=$ $e^{\phi_{\beta}\left(\bar{e}_{\beta}\right)}$, with

$$
\phi_{\beta}(e)=\log p_{\beta}(e)-i \eta e+\eta^{2} C(e) / 2+\log \left(\frac{2 \pi C(e)}{2}\right)
$$

where $C^{-1}=\partial_{e}^{2} \log p_{\beta_{0}}(e)$. However, if $\ddot{W}_{\beta_{0}}(e)$ has a curvature anomaly it exists a range of $\beta$ for which the equation $\partial_{e} \log \left(\bar{W}_{\beta_{0}}(e)\right)-\left(\beta-\beta_{0}\right)=0$ has three solutions $e_{1}, e_{2}$ and $e_{3}$. Two of these extrema are maxima so that we can use a double saddle point approximation which will be valid close to thermodynamical limit[29]

$$
z_{\beta}=e^{\phi_{\beta}\left(e_{1}\right)}+e^{\phi_{\beta}\left(e_{3}\right)}=2 e^{\phi_{\beta}^{+}} \cosh \left(\phi_{\beta}^{-}\right)
$$

where $2 \phi_{\beta}^{+}=\phi_{\beta}\left(e_{1}\right)+\phi_{\beta}\left(e_{3}\right)$ and $2 \phi_{\beta}^{-}=\phi_{\beta}\left(e_{1}\right)-\phi_{\beta}\left(e_{3}\right)$. The zeros of $Z_{\beta}$ then correspond to $\phi_{\beta}^{\vec{\beta}}=i(2 n+1) \pi$. The imaginary part is given by $\eta=$ $2(2 n+1) \pi /\left(e_{3}-e_{1}\right)$ while for the real part we should solve the equation $\Re\left(\phi_{\gamma}^{-}\right)=$ 0 . In particular, close to the real axis this equation defines a $\beta$ which can be taken as $\beta_{0}$. If the bimodal structure persists when the number of particles goes to infinity, the loci of zeros corresponds to a line perpendicular to the real axis with a uniform distribution as expected for a first order phase transition.

This demonstrates the link of the curvature anomalies of thermodynamical potentials which are equivalent to bimodalities of probability distribution with 
the usual definition of first order phase transitions when the thermodynamical limit is reached.

\subsection{The death of thermodynamics}

Let us now investigate the more general situation when the system size goes to infinity even if the Van Hove theorem do not apply. The microcanonical caloric curve in the phase transition region may either converge towards the Maxwell construction or keep a backbending behavior, since a negative heat capacity system can be thermodynamically stable even in the thermodynamical limit if it is isolated (see chapter 5). In particular we have shown in chapter 5 that if the interaction is long ranged the topological anomaly leading to the convex intruder in the entropy is not cured by increasing the number of particles[21],[31],[32].

Within our approach based on the topology of the probability distribution of observables [26] we have just shown that ensemble inequivalence arises from fuctuations of the order parameter. Ensembles putting different constraints on the fluctuations of the order parameter leads to different thermodynamics. In the case of phase transitions with non-zero latent heat, the total energy usually plays the role of an order parameter except in the microcanonical ensemble. Therefore, the microcanonical ensemble which forbids energy fluctuation is expected to presents a different thermodynamics than the other (canonical) ensembles. This different behavior may remain at the thermodynamical limit depending upon the specific properties of the considered system. In such a case, it may happen that the energy of a subsystem may become an order parameter when the total energy is constrained by a conservation law or a microcanonical sorting. This frequently occurs for Hamiltonians containing a kinetic energy contribution: if the kinetic heat capacity is large enough we will now show that the kinetic energy becomes an order parameter in the microcanonical ensemble. This is almost a paradox since in any other ensembles in which no total energy conservation is imposed the kinetic energy has a trivial perfect gas behavior while in the microcanonical ensemble it becomes an order parameter with the specific bimodal structure at the phase transition. Then, the microcanonical caloric curve presents at the thermodynamical limit a temperature jump in complete disagreement with the canonical ensemble.

Let us consider a finite system for which the Hamiltonian can be separated into two components $E=E_{1}+E_{2}$, that are statistically independent $\left(W\left(E_{1}, E_{2}\right)=W_{1}\left(E_{1}\right) W_{2}\left(E_{2}\right)\right)$ and such that the associated degrees of freedom scale in the same way with the number of particles; we will also consider the case where $S_{1}=\log W_{1}$ has no anomaly while $S_{2}=\log W_{2}$ presents a convex intruder which is preserved at the thermodynamical limit $(V \rightarrow \infty, N \rightarrow \infty$ with $N / V=c s t)$. Typical examples of $E_{1}$ are given by the kinetic energy for a classical system with velocity independent interactions. For other similar one-body operators see ref. [31] .

The probability to get a partial energy $E_{1}$ when the total energy is $E$ is given by 


$$
P_{E}\left(E_{1}\right)=\exp \left(S_{1}\left(E_{1}\right)+S_{2}\left(E-E_{1}\right)-S(E)\right)
$$

The extremum of $P_{E}\left(E_{1}\right)$ is obtained for the partitioning of the total energy $E$ between the kinetic and potential components that equalizes the two partial temperatures $\bar{T}_{1}^{-1}=\partial_{E_{1}} S_{1}\left(\bar{E}_{1}\right)=\partial_{E_{2}} S_{2}\left(E-\bar{E}_{1}\right)=\bar{T}_{2}{ }^{-1}$. If $\bar{E}_{1}$ is unique, $P_{E}\left(E_{1}\right)$ is mono-modal and we can use a saddle point approximation around this solution to compute the entropy $S(E)=\log \int_{-\infty}^{E} d E_{1} \exp \left(S_{1}\left(E_{1}\right)+S_{2}\left(E-E_{1}\right)\right)$ At the lowest order, the entropy is simply additive so that the microcanonical temperature of the global system $\partial_{E} S(E)=\bar{T}^{-1}$ is the one of the most probable energy partition. Therefore, the most probable partial energy $\bar{E}_{1}$ acts as a microcanonical thermometer. If $\bar{E}_{1}$ is always unique, the kinetic thermometer in the backbending region will follow the whole decrease of temperature as the total energy increases. Therefore, the total caloric curve will present the same anomaly as the potential one.

If conversely the partial energy distribution is double humped, then the equality of the partial temperatures admits three solutions one of them $\bar{E}_{1}^{(0)}$ being a minimum. At this point the partial heat capacities $C_{1}^{-1}=-\bar{T}^{2} \partial_{E_{1}}^{2} S_{1}\left(\bar{E}_{1}^{(0)}\right)$ and $C_{2}^{-1}=-\bar{T}^{2} \partial_{E_{2}}^{2} S_{2}\left(E-\bar{E}_{1}^{(0)}\right)$ fulfill the relation

$$
C_{1}^{-1}+C_{2}^{-1}<0
$$

This happens when the potential heat capacity is negative and the kinetic energy is large enough $\left(C_{1}>-C_{2}\right)$ to act as an approximate heat bath: the partial energy distribution $P_{E}\left(E_{1}\right)$ in the microcanonical ensemble is then bimodal as the total energy distribution $P_{\beta}(E)$ in the canonical ensemble. The bimodality of $P_{E}\left(E_{1}\right)$ implies that the kinetic energy is an order parameter of the transition in the microcanonical ensemble. In this case, performing a double saddle point approximation around the two maxima leads to a microcanonical temperature given by a weighted average of the two estimations from the two maxima of the kinetic energy distribution

$$
T=\partial_{E} S(E)=\frac{\bar{P}^{(1)} \sigma^{(1)} / \bar{T}^{(1)}+\bar{P}^{(2)} \sigma^{(2)} / \bar{T}^{(2)}}{\bar{P}^{(1)} \sigma^{(1)}+\bar{P}^{(2)} \sigma^{(2)}}
$$

where $\bar{T}^{(i)}=T_{1}\left(\bar{E}_{1}^{(i)}\right)$ are the kinetic temperatures calculated at the two maxima, $\bar{P}^{(i)}=P_{E}\left(\bar{E}_{1}^{(i)}\right)$ are the probabilities of the two peaks and $\sigma^{(i)}$ their widths. At the thermodynamical limit eq.(48) reads $c_{1}^{-1}+c_{2}^{-1}<0$, with $c=$ $\lim _{N \rightarrow \infty} C / N$. If this condition is fulfilled the probability distribution $P_{\beta}(E)$ presents two maxima for all finite sizes and only the highest peak survives at $N=\infty$. Let $E_{t}$ be the energy at which $P_{E_{t}}\left(\bar{E}^{(1)}\right)=P_{E_{t}}\left(\bar{E}^{(2)}\right)$. Because of eq.(49) at the thermodynamical limit the caloric curve will follow the high (low) energy maximum of $P_{E}\left(E_{1}\right)$ for all energies below (above) $E_{t}$; there will be a temperature jump at the transition energy $E_{t}$. 


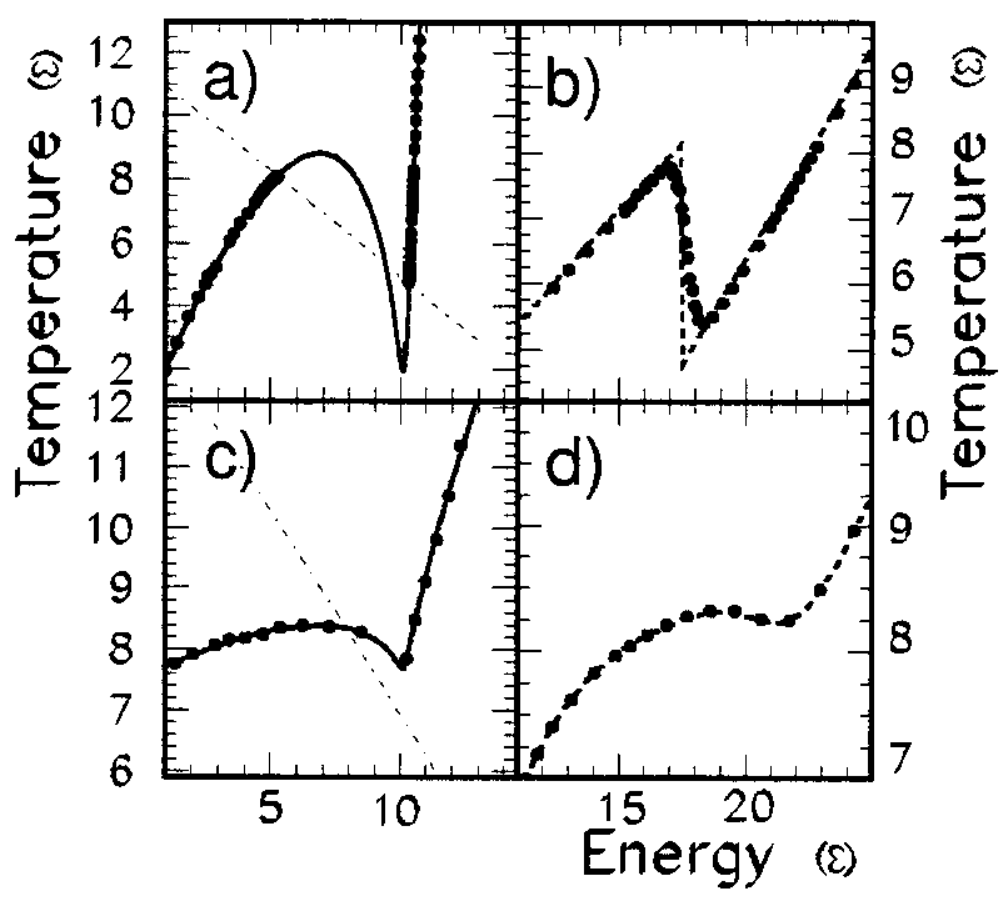

Fig. 25. Left panels: temperature as a function of the potential energy $E_{2}$ (full lines) and of the kinetic energy $E-E_{2}$ (dot-dashed lines) for two model equation of states of classical systems showing a first order phase transition. Symbols: temperatures extracted from the most probable kinetic energy thermometer from eq.(5). Right panels: total caloric curves (symbols) corresponding to the left panels and thermodynamical limit of eq.(7) (dashed lines).

This patent violation of ensemble equivalence means that, contrary to the physical intuition based on macro-systems, the equations of state are expected to explicitly depend on the characteristics of the considered ensemble of events i.e. the state variables: the fluctuating observables and the conserved quantities imposed by the dynamics or by the sorting variables used in the data analysis. This implies the impossibility to define a unique thermodynamics, i.e. a unique EoS, for systems undergoing a first order phase transition.

Let us illustrate the above results with two examples for a classical gas of interacting particles. For the kinetic energy contribution we have $S_{1}(E)=$ $c_{1} \ln (E / N)^{N}$ with a constant kinetic heat capacity per particle $c_{1}=3 / 2$. For the potential part we will take two polynomial parametrizations of the interaction caloric curve presenting a back bending which are displayed in the left part of figure 25. If the decrease of the partial temperature $T_{2}\left(E_{2}\right)$ is steeper than $-2 / 3$ (figure 25a ) [21] eq.(48) is verified and the kinetic caloric curve $T_{1}\left(E-E_{1}\right)$ (dot-dashed line) crosses the potential one $T_{2}\left(E_{2}\right)$ (full line) in three different points for all values of the total energy lying inside the coexistence region. The 


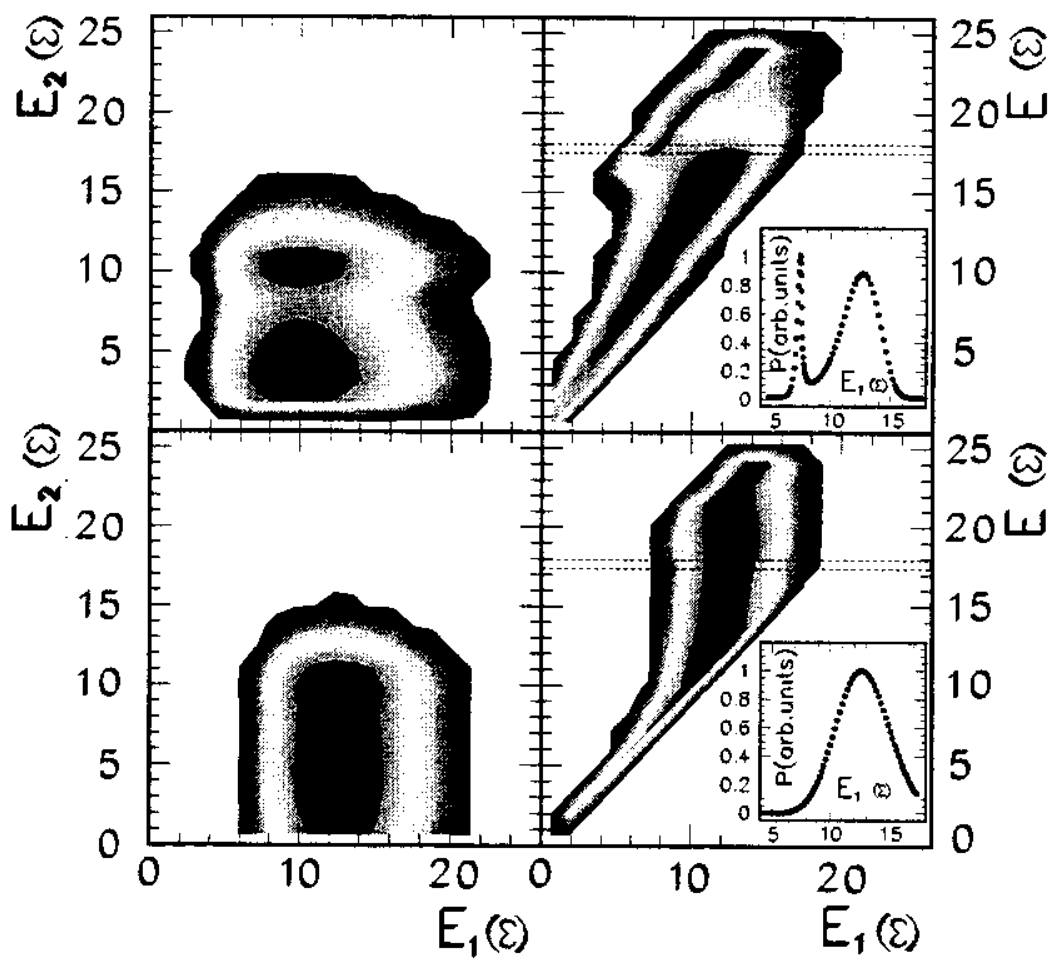

Fig. 26. Canonical event distributions in the potential versus kinetic energy plane (left panels) and total versus kinetic energy plane (right panels) at the transition temperature for the two model equations of state of figure 1. The inserts show two constant total energy cuts of the distributions.

resulting caloric curve for the whole system is shown in figure $25 \mathrm{~b}$ (symbols) together with the thermodynamical limit (lines) evaluated from the double saddle point approximation (49). In this case one observes a temperature jump at the transition energy. If the temperature decrease is smoother (figure 25c) the shape of the interaction caloric curve is preserved at the thermodynamical limit (figure $25 \mathrm{~d})$.

The occurrence of a temperature jump in the thermodynamical limit is easily spotted by looking at the bidimensional canonical event distribution $P_{\beta}\left(E_{1}, E_{2}\right)$ in the partial energies plane. This density of states is just the product of the independent kinetic and potential canonical probabilities as shown in the left part of figure 26 for the two model equation of states of figure 25 at the transition temperature $\beta=\beta_{t}$. In order to discuss the microcanonical ensemble one has to introduce the total energy $E=E_{1}+E_{2}$. Keeping $E$ and $E_{1}$ as variables instead of $\left(E_{1}, E_{2}\right)$ is nothing but a simple coordinate change with unit Jacobian. Thus we can look at the canonical distribution as a function of $E$ and $E_{1}, P_{\beta}\left(E, E_{1}\right) \propto$ $\exp S_{1}\left(E_{1}\right) \exp S_{2}\left(E-E_{1}\right) \exp (-\beta E)$ which is shown in the right part of figure 

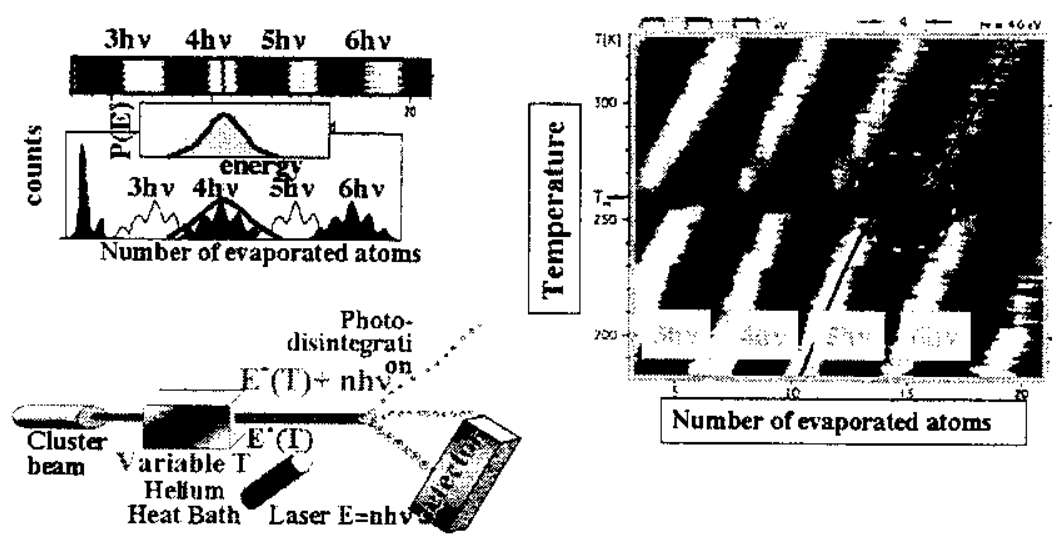

Fig. 27. Left bottom: a schematic picture of the experimental set-up. Left top: illustration of the shift of the cluster internal energy distribution by the photons' energy leading to a measurable distribution of evaporated fragments. Right: the observed correlation between the temperature of the Helium bath and the fragmentation pattern induced by the absorption of several photons. The "accident" at a given temperature indicates the melting point [33].

26. The deformation of the event distribution induced by the microcanonical constraint does not cause a topological difference between our two model cases; this explains why both converge to the Maxwell construction for $N \rightarrow \infty$ in the canonical ensemble. If we now study the microcanonical ensemble we have to look at constant energy cuts of $P_{\beta}\left(E, E_{1}\right)$ leading to the microcanonical distribution $P_{E}\left(E_{1}\right)$ within a normalization constant. If the anomaly in the potential equation of state is sufficiently important, the distortion of events is such that one can still see the two phases coexist even after a sorting in energy as shown in the same figure 26 for two cuts of $P_{\beta}\left(E, E_{1}\right)$ at an energy close to the transition energy.

\section{Observables: melting of metallic clusters [33]}

In the year 2000 the first experimental signature of a back bending caloric curve has been reported in the melting of metallic clusters. The experiment is rather simple. The clusters are first produced and selected. Then the clusters get thermalized in the melting temperature region in an helium heat bath. After thermalization they are further excited by a laser beam absorbing several photons, thanks to the plasmon vibration. The average energy is then such that clusters have time to evaporate atoms within the experiment time scale. The number of evaporated atoms provides a measure of the cluster excitation energy. Since the photon energy is fixed, this is a measure of the excitation energy at the exit of the heat bath. Changing the temperature the thermal excitation changes and the distribution of evaporated atoms is shifted. The obtained bidimensional pictures 

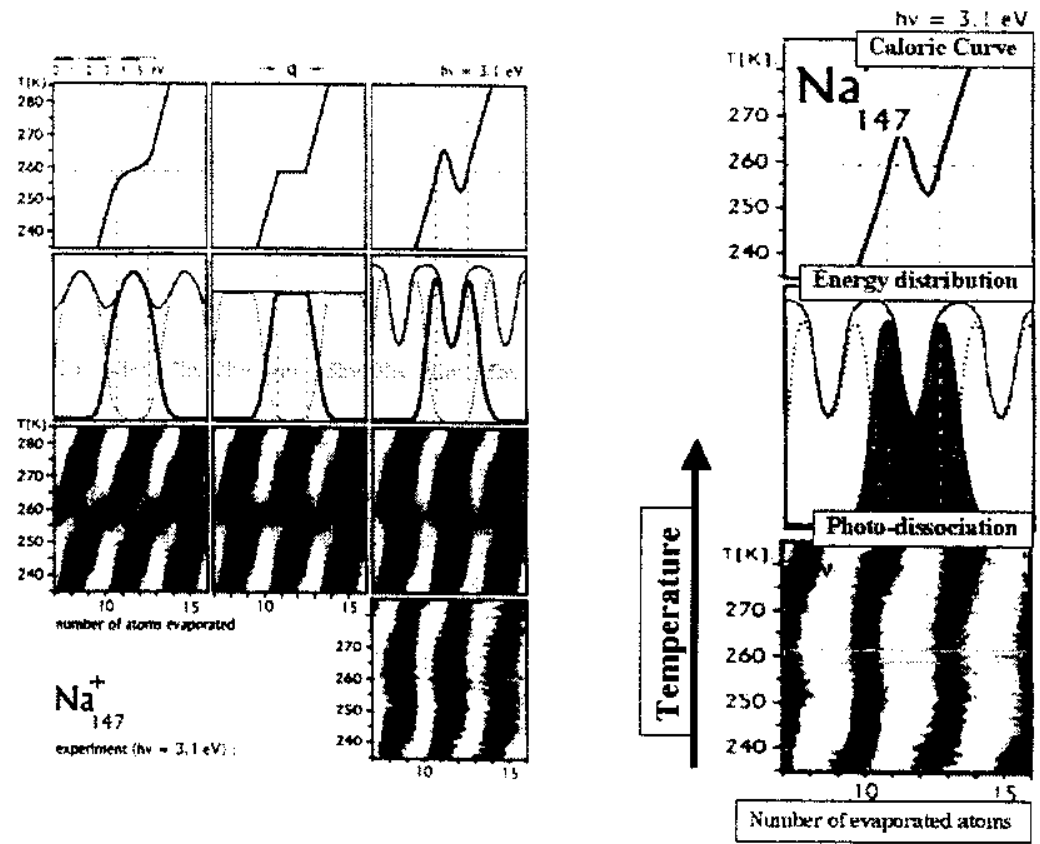

Fig. 28. Left side: several scenarii for the transition between two phases, a broad monomodal energy distribution, a flat one and a bimodal one, are compared with the experimental results. The observed pattern (last row) is only compatible with the existence of a bimodal energy distribution. Right side: enlarged diagram indicating the presence of a bimodality which looks like a sudent jump in the energy distribution. [33].

of the number of evaporated atoms as a function of the oven temperature clearly show an anomaly corresponding to the melting point. (see figure 27).

Instead of looking for a back bending of the microcanonical caloric curve the authors of reference [33] have tried to observe the associated bimodal canonical energy distribution. Since the number of evaporated atoms is a measure of the cluster excitation energy before the photons' absorption, this distribution is a measure of the canonical distribution of excitation energy. The difference between a back bending and a monotonous caloric curve is that the energy distribution has a bi-modal or a mono-modal shape (see chapter 6 ). This induces a modification of the fragmentation pattern. The only difficulty is that the system may absorb different numbers of photons. Therefore one observe replicas of the same distribution every $h \nu$. The trick is thus to correctly tune the laser energy so that it will not blur the jump. On can see in figure28 that the observed pattern is only compatible with a negative heat capacity system. The results can be interpreted if one assumes a 10 degree decrease of the temperature at the phase transition energy. 


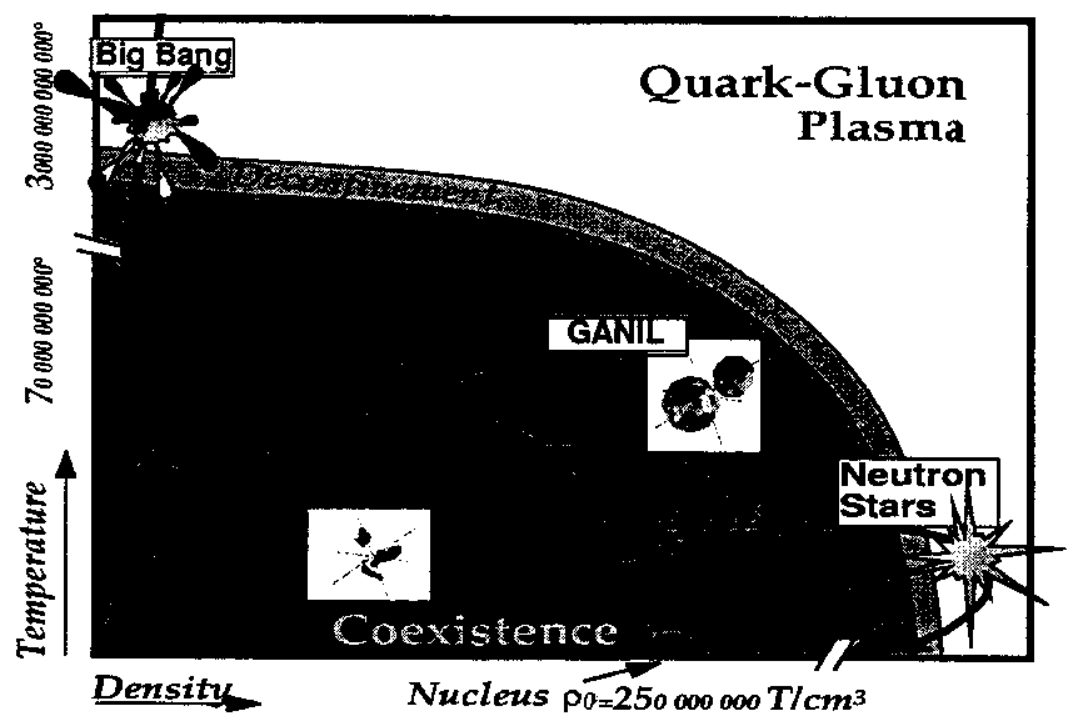

Fig. 29. Nuclear Phase diagram in the temperature density plane. At low temperature and below the saturation density a liquid gas phase transition is expected.

\section{Observables: the nuclear physics case}

\subsection{Liquid gas phase transition in nuclear systems}

Since nuclear forces resemble to Van der Waals interactions the nuclear phase diagram (see figure 29) is expected to present a liquid gas phase transition. Our present knowledge of the nuclear equation of state is limited. The main reason is the difficulty to treat the nuclear many-body problem and to define a reliable in medium interaction. The saturation energy and density, i.e. the ground state of nuclear matter, are well established but the compressibility, i.e. the variation of the energy as a function of the density around the saturation point, is still under discussion. As far as the temperature dependence of nuclear properties is concerned very little is also know in an absolute way. Only the entropy variation, i.e. the level density parameter $a=S / T$, of a finite nucleus as an open system has been clearly established through evaporation studies.

An important research activity is now devoted to the extraction of reliable information of the nuclear equation of states and the associated phase diagram. Heavy ion reactions are routinely used to test mechanical and thermodynamical properties of nuclei. In particular in the recent years the multifragmentation regime has been tentatively associated with the occurrence of a liquid-gas phase transition. 


\subsection{Negative heat capacities and abnormal fluctuations}

At the same time of the observation of negative heat capacity in the melting transition (see chapter 7), a $C<0$ signal has also been reported for the nuclear multifragmentation transition using the fluctuations of the energy partition [34].

The investigation method [35] can be easily explained for a classical fluid and tested in the framework of the lattice-gas model. The total energy $E$ of the considered system can be decomposed into two independent components, its kinetic and potential energy: $E=E_{k}+E_{p}$. In a microcanonical ensemble with a total energy $E$ the total degeneracy factor $W(E)=\exp (S(E))$ is thus simply given by the folding product of the individual degeneracy factors $W_{i}\left(E_{i}\right)=$ $\exp \left(S_{i}\left(E_{i}\right)\right)$ of the two subsystems $i=k, p$. One can then define for the total system as well as for the two subsystems the microcanonical temperatures $T_{i}$ and the associated heat capacities $C_{i}$. If we now look at the kinetic energy distribution when the total energy is $E$ we get

$$
P_{k}^{E}\left(E_{k}\right)=\exp \left(S_{k}\left(E_{k}\right)+S_{p}\left(E-E_{k}\right)-S(E)\right)
$$

Using Eq. (50) we directly get that the most probable partitioning of the total energy $E$ between the potential and kinetic components is characterized by a unique microcanonical temperature $\bar{T} \equiv T_{k}\left(\bar{E}_{k}^{E}\right)=T_{p}\left(E-\bar{E}_{k}^{E}\right)$. Therefore the most probable kinetic energy $\bar{E}_{k}^{E}$ can be used as a microcanonical thermometer as shown in Figure 30 . Using a Gaussian approximation for $P_{k}^{E}\left(E_{k}\right)$ the kinetic energy variance can be calculated as [35]

$$
\sigma_{k}^{2}=\bar{T}^{2} \frac{C_{k} C_{p}}{C_{k}+C_{p}}
$$

where $C_{k}$ and $C_{p}$ are the microcanonical heat capacities calculated for the most probable energy partition.

As shown in Figure 30 when $C_{p}$ diverges and then becomes negative, $\sigma_{k}^{2}$ remains positive but overcomes the canonical expectation $\sigma_{k}^{2}=\bar{T}^{2} C_{k}$. This anomalously large kinetic energy fluctuation is a signature of the first order phase transition. Equation (51) can be inverted to extract from the observed fluctuations the heat capacity

$$
C \simeq C_{k}+C_{p}=\frac{\bar{T}^{2} C_{k}^{2}}{\bar{T}^{2} C_{k}-\sigma_{k}^{2}}
$$

Figure 30 shows that the heat capacity extracted from the kinetic energy fluctuations is in very good agreement with the exact one. This means that kinetic energy fluctuations are an experimentally accessible measure of the heat capacity which allows to sign divergences and negative branches characteristic of the phase transition.

\subsection{Experimental results}

The negative heat capacity signal of a phase transition has been looked for in experiments. In such a case an easy splitting of the energy is between the thermal 

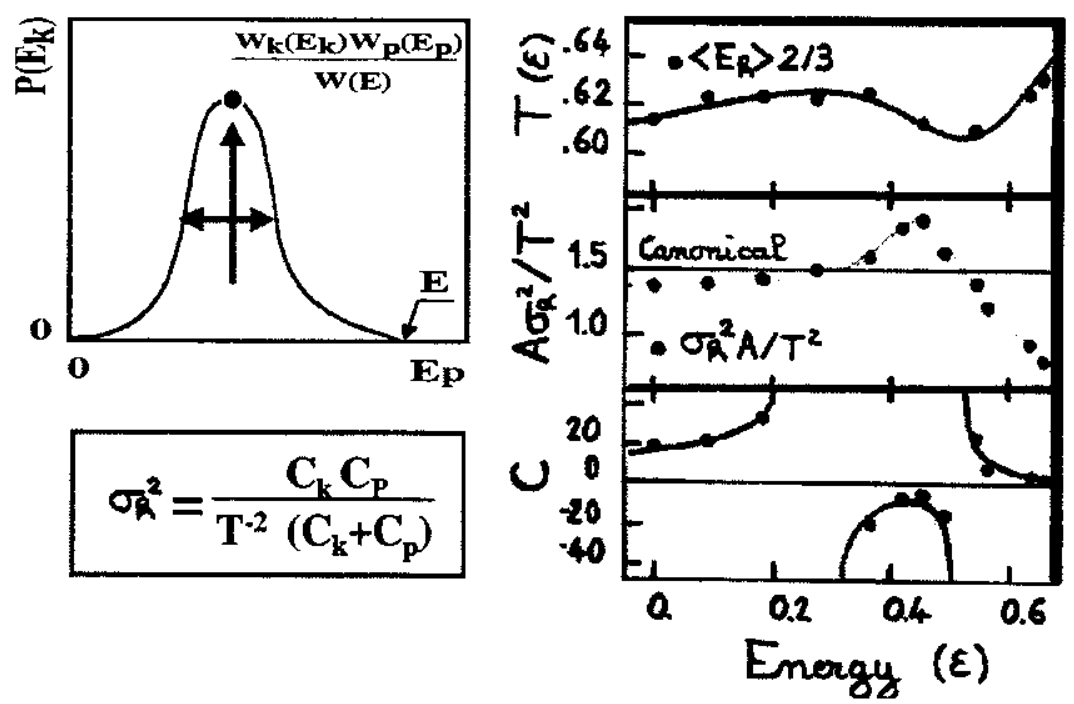

Fig. 30. Left part: the schematic distribution of partial energy for a fixed total energy. Right: the comparison of the various measurements (dots) with the exact results of the lattice-gas model (lines).

excitation and agitation on one side and the partition Q-value plus the Coulomb interaction on the other side. The expected canonical prediction can be inferred from the relation between the average kinetic energy and the temperature since this provides $C_{k}$. Figures 31 and 32 show the first experimental results of a fluctuation overcoming the canonical expectation with the corresponding deduced heat capacity for excited nuclei in the gold mass range [34].

It is important to know that these measurements need a very sophisticated and accurate technique to correctly reconstruct the configurational energy fluctuations at the time of fragment formation. These reconstructions often need hypotheses such as the volume of the freeze-out and the origin of emitted particles. Additional measurements to control these hypotheses have to be performed. However, kinetic energy fluctuations are a very promising way to infer thermodynamical properties and to signal phase transitions.

\section{References}

1.

2. E.T.Jaynes, Phys.Rev.106(1957)620 and Phys.Rev.108(1957)171

3. A.Katz, 'Principles of statistical mechanics'F reeman (1967)

4. S.Abe, Y.Okamoto, 'Nonextensive statistical mechanics and its applications', Lecture Notes in Physics vol.560 (2001)

5. R.Balian, 'From microphysics to macrophysics', Springer Verlag (1982)

6. Ph. Chomaz, Ann. Phys. Fr. 21 (1996) 669 


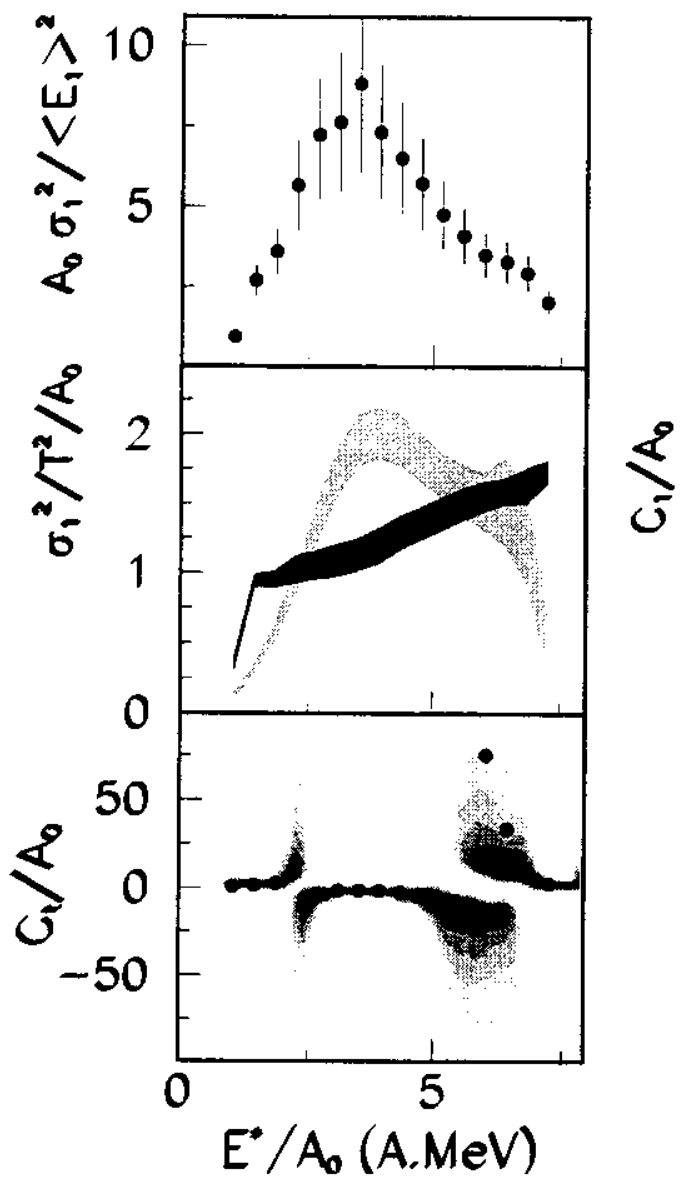

Fig. 31. Configurational energy fluctuations (top), comparison with the canonical expectation (middle) and deduced heat capacity as a function of the excitation energy (bottom) for the $\mathrm{Au}$ quasi-projectile formed in $\mathrm{Au}+\mathrm{Au}$ collisions at $35 \mathrm{MeV} /$ nucleon. Multics-Miniball data from ref.[34]

7. L.Onsager, Phys.Rev.65 (1944) 117

8. C.N.Yang, Phys.Rev.85 (1952) 809

9. E.A.Guggenheim, J.Chem.phys.13 (1945) 253.

10. L.D.Landau, E.M.Lifshitz, 'Statistical Physics', Pergamon Press (1980)

11. M.E.Fisher,'Critical phenomena', Academic (1971)

12. Ph.Chomaz, F.Gulminelli, Phys.Rev.Lett.82 (1999)1402

13. Ph.Chomaz, F.Gulminelli, Int.Journ.Mod.Phys.E8 (1999) 527

14. M.S.Challa, J.H.Hetherington, Phys.Rev.Lett.60 (1988)77 and Phys.Rev.A38 (1988) 6324

15. D.H.E.Gross, Phys.Rep.279 (1997)119 and "Microcanonical thermodynamics: phase transitions in finite systems", Lecture notes in Physics vol.66, World Scientific (2001)

16. F. Gulminelli, Ph.Chomaz, Europhys.Lett.50 (2000) 434

17. F.Gulminelli, Ph.Chomaz, Phys.Rev.Lett.85 (2000) 3587 

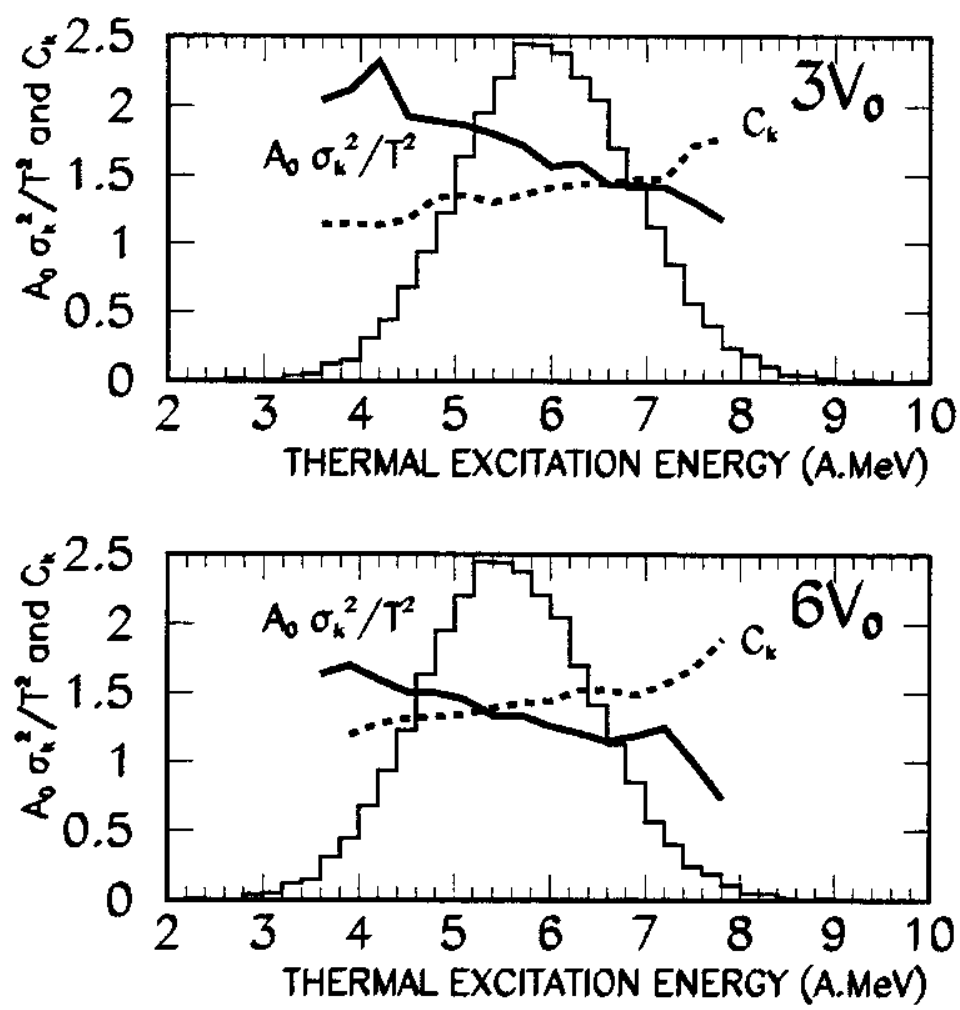

Fig. 32. Configurational energy fluctuations (full line) compared to the canonical expectation (dashed line) and excitation energy distribution (histogram) for central Xe+Sn collisions at $32 \mathrm{MeV} /$ nucleon. The two pictures correspond to two different hypotheses in the data reconstruction. Indra data from ref.[34]

18. T.L.Beck, R.S.Berry, Journ.Chem.Phys. 88 (1988) 3910

19. L.Moretto et al., Phys.Rep.287 (1997) 249

20. V.Antonov, Vest.Leningrad Univ.7(1962)135

21. D.Lynden-Bell, Monthly Not.Astr.Soc. 138 (1968) 495

22. W.Thirring, Z.Phys.235 (1970) 339

23. T.Padmanabhan, Phys.Rep.188 (1990) 285

24. K. Binder, D. P. Landau, Phys. Rev. B 30 (1984) 1477.

25. P. Labastie and R. L. Whetten, Phys. Rev. Lett. 65 (1990) 1567.

26. Ph. Chomaz, F. Gulminelli and V. Duflot, Phys. Rev. E 64 (2001) 046114 and arXiv:cond-mat/0112222.

27. T.Poston, I.Stewart, "Catastrophe Theory and its applications", Pitman (1978)

28. C.N.Yang and T.D.Lee, Phys.Rev.87 (1952) 404.

29. K.C. Lee, Phys. Rev. 53 E (1996) 6558.

30. M.S.S.Challa et al. Phys. Rev. 34B (1986) 1841. 
31. J. Barré, D. Mukamel and S. Ruffo, Phys. Rev. Lett.87 (2001)030601-1.

32. L. Casetti, M. Pettini and E. G. D. Cohen, Phys. Rep. 337 (2000) 237.

33. M. Schmidt et al, Nature 393 (1998) 238 and Phys.Rev.Lett.86 (2001) 1191

34. M. D'Agostino et al., Nucl.Phys. A 650 (1999) 329, Phys.Lett.B 473 (2000) 219, Nucl.Phys.A 699 (2002)795.

35. P.Chomaz and F.Gulminelli, Nucl. Phys. A647 (1999) 153. 